Supporting Information for:

\title{
Examination of Molten Salt Reactor Relevant Elements Using Hydrothermal Synthesis
}

Cory J. Windorff, Alexander T. Chemey, Joseph M. Sperling Bonnie E. Klamm, and Thomas E. Albrecht-Schmitt*

Department of Chemistry and Biochemistry, Florida State University, 95 Chieftan Way, RM.

118 DLC, Tallahassee, Florida 32306, United States.

*talbrechtschmitt@fsu.edu

$\underline{\text { Table of Contents }}$

EXPERIMENTAL DETAILS

S2-S8

UV/VIS/NIR SPECTROSCOPY AND PHOTOGRAPHS OF CRYSTALS

$\mathbf{S 8}-\mathbf{S 2 1}$

BOND COMPARISONS

S22-S23

Crystallographic Details

S23-S49

REFERENCES

S49 - S51 


\section{EXPERIMENTAL DETAILS}

CAUTION! Hydrofluoric acid is an acutely toxic chemical. All manipulations were performed with appropriate safety precautions: manipulations were performed in a well ventilated fume hood behind the sash while wearing heavy weight natural latex gloves with a second pair of nitrile gloves over them and a tube of calcium gluconate within arm's reach. HF waste was

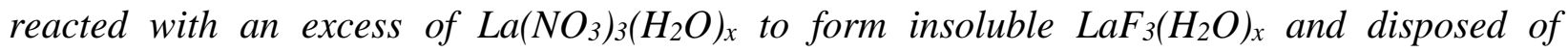
accordingly.

CAUTION! Beryllium is a highly toxic metal, which can cause berylliosis in time or death upon acute inhalation and is a suspected carcinogen. To prevent exposure researchers wore dust masks and two sets of nitrile gloves, with the inner pair taped to the lab coat such that no skin is exposed, while handling open powders. Samples were transferred to a welll ventilated fume hood for further manipulations. To minimize contamination surfaces were coated in aluminum foil and were wiped clean using alcohol coated task wipes (Kim-wipes), all of the exposed items (foil, wipes and gloves) were disposed in proper waste containers.

CAUTION! ${ }^{237} N p\left(t_{1 / 2}=2.14 \times 10^{6}\right.$ years $)$ is a serious health threat due to its high energy $\alpha$ (4.788 MeV) emitting isotope with high energy $\alpha$-, $\beta$ - and $\gamma$-emissions from its daughters. All studies with ${ }^{237} \mathrm{~Np}$ were conducted in a radiation laboratory equipped with a High-Efficiency Particulate Air (HEPA) filtered hood. All free-flowing solids were handled in negative pressure gloveboxes or under heavy oil.

Materials. $\mathrm{MeOH}$ (reagent grade, Sigma) $\mathrm{HF}_{(\mathrm{aq})}\left(48\right.$ wt \% in $\mathrm{H}_{2} \mathrm{O}$, Sigma), $\mathrm{LiF}$ (99.85\%, Alfa Aesar), NaF (99.99\% Alfa Aesar) BeO (99\%, Alfa Aesar), $\mathrm{ZrO}_{2}$ (99\% $5 \mu \mathrm{m}$ particle size, Sigma), $\mathrm{CeO}_{2}$ (G. Fredrick Smith Chemical Co.), $\mathrm{Nd}_{2} \mathrm{O}_{3}$ (99.9\%, Sigma), $\mathrm{ThO}_{2}$ (99.99\%, Strem), $\mathrm{UO}_{2}\left(99.8 \%\right.$, Strem), $\mathrm{UO}_{3} \cdot 2 \mathrm{H}_{2} \mathrm{O}\left(99.8 \%\right.$, Strem) ${ }^{237} \mathrm{NpO}_{2}$ (Oak Ridge National Laboratory) were 
used as received without further purification. Deionized water was used directly from the house DI tap without further action.

Instrumentation. UV/vis/NIR: Single crystal UV/vis/NIR measurements were recorded using a CRAIC microphotospectrometer on crystals placed on a borosilicate glass slide in Cargile Immersion oil ${ }^{\circledR}$ and recorded from 320 to $1700 \mathrm{~nm}$. SCXRD: Single crystals selected for data collection were mounted on a MITOGEN mount ${ }^{\mathrm{TM}}$ cryoloops and optically aligned on a Bruker D8 Quest X-ray diffractometer. Crystallographic data were collected under a low-temperature nitrogen gas flow using a Mo-K $\mathrm{K}_{\alpha}(\lambda=0.71069 \AA) \mathrm{I} \mu \mathrm{X}$-ray micro source and a CMOS detector. The unit cell determination and subsequent data collection was performed using the APEX III software. ${ }^{1}$ Raw data frames were processed using SAINT $^{2}$ and SADABS. $^{3}$ Subsequent calculations were carried out using the SHELXL or OLEX 2 programs. $^{4-7}$ Compound Collections: $\mathbf{L i N p F 5}$, a pale green needle of approximate dimensions $0.551 \times 0.074 \times 0.049 \mathrm{~mm}$ was mounted on a cryoloop and after a unit cell determination was found to isomorphous with its $\mathrm{U}^{8-9}$ analog. A $20 \mathrm{sec}$ frame collection with a scan width of $0.3^{\circ}$ was utilized to collect a hemisphere of data at a detector distance of $35 \mathrm{~mm}$ at a temperature of $100 \mathrm{~K}$. $\mathbf{L i}_{4} \mathbf{C e F}_{8}$, a colorless plate shaped crystal of approximate dimensions $0.282 \times 0.120 \times 0.094 \mathrm{~mm}$ was mounted on a cryoloop and after a unit cell determination was found to be isomorphous with its $\mathrm{U}^{10}$ and $\mathrm{Tb}^{11}$ analogs. A 5 second frame collection with a scan width of $0.7^{\circ}$ was utilized to collect a hemisphere of data at a detector distance of $50 \mathrm{~mm}$ at a temperature of $120 \mathrm{~K}$. The structure features a small disorder of the Ce atom with a secondary position half the distance to the next unit cell as well as disorder of the $\mathrm{Li}^{+}$ions which cannot be satisfactorily modeled, both situations are similar to what is observed in its $U$ analog. ${ }^{10} \mathbf{L i}_{6} \mathbf{B e F}_{4} \mathbf{Z r F}_{\mathbf{8}}$, a block shaped colorless crystal of approximate dimensions $0.28 \times 0.24 \times 0.18 \mathrm{~mm}$ was mounted on a cryoloop 
and after a unit cell determination was found to isomorphous with its previous collection. The crystal was recollected to obtain high quality modern data. ${ }^{12}$ A 2 second frame collection with a scan width of $0.3^{\circ}$ was utilized to collect a hemisphere of data at a detector distance of $50 \mathrm{~mm}$ at a temperature of $120 \mathrm{~K} . \mathbf{N p}_{3} \mathbf{F}_{12}\left(\mathbf{H}_{2} \mathbf{O}\right)$, a needle shaped pale green crystal of approximate dimensions $0.008 \times 0.005 \times 0.003 \mathrm{~mm}$ was mounted on a cryloop and found to be isomorphous with its previous collection and its $\mathrm{U}$ analog. ${ }^{13-14}$ The crystal was recollected to see if a higher quality collection could be achieved. A 45 second frame collection with a scan width of $0.5^{\circ}$ was utilized to collect a hemisphere of data at a detector distance of $60 \mathrm{~mm}$ at a temperature of 100 $\mathrm{K}$. The data The structure was previously characterized in the Monoclinic $\mathrm{Cm}$ space group $[\beta=$ 128.77(2) ${ }^{\circ}$, however, the current preference of CheckCIF for the non-standard $I$-centering was utilized instead and the structure was solved in the $\operatorname{Im}$ space group $\left[\beta=95.546(2)^{\circ}\right]$. The structure was solved as an enantiomeric twin, and due to weak diffraction the hydrogen atoms on the coordinated water molecules was not located in the Fourier map and were instead added based on calculated positions. $\mathrm{Ce}_{3} \mathbf{F}_{12}\left(\mathrm{H}_{2} \mathrm{O}\right)$, a block shaped colorless crystal of approximate dimensions $0.242 \times 0.164 \times 0.090 \mathrm{~mm}$ was mounted on a cryloop and found to be isomorphous with its $\mathrm{U}^{14}$ and $\mathrm{Np}^{13}$ analogs. A 1 second frame collection with a calculated scan of data at a detector distance of $60 \mathrm{~mm}$ at a temperature of $100 \mathrm{~K}$. The data The can be characterized in the Monoclinic $\mathrm{Cm}$ space group $\left[\beta=128.817(1)^{\circ}\right]$, however, the current preference of CheckCIF for the non-standard $I$-centering was utilized instead and the structure was solved in the $I m$ space group $\left[\beta=95.607(1)^{\circ}\right]$. The structure was solved as an enantiomeric twin, the high quality data allowed the location of the hydrogen atoms on the coordinated water molecules in the Fourier map, as was observed in the $\mathrm{U}$ analog. ${ }^{14}$ 
General Synthesis FLi(Be)M. LiF ( 38 mg, $\sim 1.5 \mathrm{mmol}), \mathrm{BeO}(\sim 11 \mathrm{mg}, \sim 0.44 \mathrm{mmol})$ and $\mathrm{MO}_{\mathrm{x}}$ ( $\sim 0.25 \mathrm{mmol}$ ) were combined in a $12 \mathrm{~mL}$ Teflon cup and moved to a fume hood where $\mathrm{DI}^{\mathrm{H}} \mathrm{H}_{2} \mathrm{O}$ (750 $\mu \mathrm{L}$ ) and $\mathrm{HF}_{(a q)}(48$ wt $\%, 500 \mu \mathrm{L})$ was added. The cup was sealed, placed inside a steal jacket, removed from the fume hood, sealed and placed inside a box furnace in a separate fume hood. The heating profiled consisted of a ramp from room temperature to $200{ }^{\circ} \mathrm{C}$ over $30 \mathrm{~min}$, a $72 \mathrm{~h}$ dwell followed by cooling to $25^{\circ} \mathrm{C}$ at a rate of $4{ }^{\circ} \mathrm{C} / \mathrm{h}$. The jacket was unsealed, the Teflon cup was removed and carefully opened in the fume hood. The supernatant was decanted, washed with $\mathrm{MeOH}(3 \times 1 \mathrm{~mL})$ and left to dry in air at least $3 \mathrm{~h}$ and $\mathrm{X}$-ray quality crystals were selected upon drying.

$\mathrm{ZrO}_{2}: 31 \mathrm{mg}(0.25 \mathrm{mmol})$, large colorless blocks were isolated and analyzed to be $\mathbf{L i}_{6} \mathbf{B e F}_{4} \mathbf{Z r F}_{\mathbf{8}}$ by crystallographic analysis. ${ }^{12}$

$\mathrm{CeO}_{2}$ : $45 \mathrm{mg}(0.26 \mathrm{mmol})$, a small amount of colorless block shaped crystals were obtained and analyzed to be $\mathbf{L i 4} \mathbf{C e F} 8$ by crystallographic analysis, along with a large amount of beige powder. $\mathrm{Nd}_{2} \mathrm{O}_{3}: 40 \mathrm{mg}(0.12 \mathrm{mmol})$, no crystalline material was isolated, only colorless powder. $\mathrm{ThO}_{2}: 40 \mathrm{mg}(0.26 \mathrm{mmol})$, no crystalline material was isolated, only colorless powder. $\mathrm{UO}_{2}: 73 \mathrm{mg}(0.27 \mathrm{mmol})$, small irregularly shaped green crystals were isolated and identified as LiUF $_{5}$ by crystallographic analysis,${ }^{8-9}$ along with large block shaped colorless crystals identified as $\mathrm{Li}_{2} \mathrm{BeF}_{4}$ by crystallographic analysis. ${ }^{8-9,}{ }^{15-18} \mathrm{UV} / \mathrm{vis} / \mathrm{NIR}$ [single crystal, exited state assignment $\left.{ }^{19}-\lambda_{\max }, \mathrm{nm}\left(\mathrm{cm}^{-1}\right),\right]:{ }^{1} \mathrm{I}_{6}+{ }^{3} \mathrm{P}_{2}-417.8(23,940), 445.9(22,430), 472.3(21,170),{ }^{3} \mathrm{P}_{1}$

- $531.2(18,830),{ }^{3} \mathrm{~F}_{4}+{ }^{1} \mathrm{D}_{2}+{ }^{3} \mathrm{P}_{0}-614.1 \mathrm{sh}(16,280), 629.4(15,890), 644.65(15,510), 651.5 \mathrm{sh}$ (15,350), ${ }^{3} \mathrm{H}_{6}-832.5(12,010), 893.0(11,200),{ }^{3} \mathrm{~F}_{3}+{ }^{1} \mathrm{G}_{4}-1085.2(9,210),{ }^{3} \mathrm{H}_{5}-1364.5(7,330)$. 
$\mathrm{UO}_{3} \cdot 2 \mathrm{H}_{2} \mathrm{O}: 78 \mathrm{mg}(0.24 \mathrm{mmol})$, large green prismatic shaped crystals were isolated and identified as LiUF5 by crystallographic analysis, ${ }^{8-9}$ along with large block shaped colorless crystals identified as $\mathbf{L i}_{2} \mathbf{B e F}_{4}$ by crystallographic analysis. ${ }^{8-9,15-18}$

$\mathrm{NpO}_{2}: 30 \mathrm{mg}(0.11 \mathrm{mmol})$, conducted with half the volume of liquids, small pale green needles identified as $\mathbf{L i N p F} 5$ were isolated, along with large colorless blocks consistent with $\mathbf{L i}_{2} \mathbf{B e F}_{4}$. $\mathrm{UV} /$ vis/NIR [single crystal, exited state assignment ${ }^{20,19,21}-\lambda_{\max }, \mathrm{nm}\left(\mathrm{cm}^{-1}\right)$ ]: Unassigned 327.4 (30,550), $344.0(29,070), 360.5(27,740), \mathrm{J}=15 / 2,3 / 2,11 / 2-402.9$ sh $(24,820), 409.9$ (24,390), $424.8(23,540), \mathrm{J}=3 / 2,13 / 2,{ }^{11} / 2,1 / 2,{ }^{9} / 2,9 / 2,7 / 2,5 / 2-438.1$ sh $(22,830), 445.9(22,430)$, 466.1 (21,460), 473.1 (21,140), 479.3 (20, 860), $497.2(20,110), \mathrm{J}=9 / 2-528.8(18,900), 538.9$ $(18,560), \mathrm{J}=9 / 2,5 / 2,5 / 2,3 / 2,7 / 2,15 / 2,{ }^{7} / 2-581.2(17,200), 594.3(16,830), 601.1(16,630), 610.3$ (16,390), $618.0(16,180), 650.7 \mathrm{sh}(15,370), 684.1(14,620), 701.5(14,250), 713.6(14,010)$, 721.1 (13,870), $751.2(13,310), 778.2$ (12,850), $795.3(12,570), 800.6(12,490), 807.3(12,390)$, 837.0 (11,950), ${ }^{4} \mathrm{I}_{13 / 2}-873.2(11,450), 892.3$ (11,210), 915.0 (10,930), $958.7(10,430),{ }^{4} \mathrm{~F}_{3 / 2}-$ 1063.9 (9,400), 1099.9 (9,090), $1140.8(8,770),{ }^{4} \mathrm{I}_{11 / 2}-1649.7(6,060), 1681.2(5,950), 1696.9$ $(5,890)$.

Isolation of $\mathrm{M}_{3} \mathrm{~F}_{12}\left(\mathrm{H}_{2} \mathrm{O}\right)$. The synthesis was carried out in a manner similar to that of FLi(Be)M, except LiF was omitted from the reaction, $\sim 18 \mathrm{mg}(\sim 0.72 \mathrm{mmol})$ of $\mathrm{BeO}$ and $\sim 0.24$ mmol of $\mathrm{MO}_{\mathrm{x}}$ was used.

$\mathrm{ZrO}_{2}: 30 \mathrm{mg}(0.25 \mathrm{mmol})$, no crystalline material was isolated, only colorless powder.

$\mathrm{CeO}_{2}: 42 \mathrm{mg}(0.24 \mathrm{mmol})$, no crystalline material was isolated, only colorless powder. When the reaction was repeated with $\mathrm{CeO}_{2}(52 \mathrm{mg}, 0.30 \mathrm{mmol}), \mathrm{LiF}(9 \mathrm{mg}, 0.35 \mathrm{mmol})$ small colorless crystals of $\mathrm{Ce}_{3} \mathbf{F}_{12}\left(\mathrm{H}_{2} \mathbf{O}\right)$ were isolated. 
$\mathrm{Nd}_{2} \mathrm{O}_{3}: 41 \mathrm{mg}(0.12 \mathrm{mmol})$, no crystalline material was isolated, only colorless powder.

$\mathrm{ThO}_{2}: 65 \mathrm{mg}(0.25 \mathrm{mmol})$, no crystalline material was isolated, only colorless powder.

$\mathrm{UO}_{3} \cdot 2 \mathrm{H}_{2} \mathrm{O}: 75 \mathrm{mg}(0.23 \mathrm{mmol})$, small irregularly shaped green crystals were isolated identified as $\mathbf{U}_{3} \mathbf{F}_{12}\left(\mathbf{H}_{2} \mathbf{O}\right)$ by crystallographic analysis. ${ }^{22-24} \mathrm{UV} /$ vis/NIR [single crystal, exited state assignment $\left.{ }^{19}-\lambda_{\max }, \mathrm{nm}\left(\mathrm{cm}^{-1}\right)\right]:{ }^{1} \mathrm{I}_{6}+{ }^{3} \mathrm{P}_{2}-422.4$ (23,670), 432.6sh (23,120), $445.1(22,470)$, 477.0 (20,970), ${ }^{3} \mathrm{P}_{1}-532.8(18,660),{ }^{3} \mathrm{~F}_{4}+{ }^{1} \mathrm{D}_{2}+{ }^{3} \mathrm{P}_{0}-618.7 \mathrm{sh}(16,160), 634.0(15,770), 660.6$ $(15,140),{ }^{3} \mathrm{H}_{6}-836.2(11,960),{ }^{3} \mathrm{~F}_{3}+{ }^{1} \mathrm{G}_{4}-1049.2(9,530),{ }^{3} \mathrm{H}_{5}-1463.9(6,830)$.

$\mathrm{NpO}_{2}$ : $30 \mathrm{mg}(0.11 \mathrm{mmol})$, conducted with half the volume of reagents, no crystalline material was isolated, following work up only pale green powder was isolated. To the pale green powder $\mathrm{NaF}(60 \mathrm{mg}, 1.4 \mathrm{mmol}), \mathrm{DI} \mathrm{H}_{2} \mathrm{O}(750 \mu \mathrm{L})$ and $\mathrm{HF}_{(\mathrm{aq})}(48 \mathrm{wt} \%, 500 \mu \mathrm{L})$ was added, the cup was resealed and heated following the same program. The reaction mixture was decanted and washed with $\mathrm{MeOH}(3 \times 1 \mathrm{~mL})$ and left to dry in air at least $3 \mathrm{~h}$ and pale green $\mathrm{X}$-ray quality crystals were selected upon drying. UV/vis/NIR [single crystal, exited state assignment ${ }^{25,19,21}-\lambda_{\max }, \mathrm{nm}$ $\left.\left(\mathrm{cm}^{-1}\right)\right]:$ Unassigned $-360.5(27,740), \mathrm{J}=15 / 2,3 / 2,11 / 2-402.1 \quad(24,870), 409.9(24,390), 421.7$ $(23,720), 428.7(23,330), \mathrm{J}=3 / 2,13 / 2,11 / 2,1 / 2,9 / 2,{ }^{9} / 2,{ }^{7} / 2,5 / 2-438.8(22,790), 473.1(21,140)$, 478.5 (20,900), $502.6(19,900), 515.7$ (19,390), 523sh (19,100), $\mathrm{J}={ }^{9} / 2-536.6(18,640), 546.6$ $(18,290), \mathrm{J}=9 / 2,5 / 2,5 / 2,{ }^{3} / 2,7 / 2,15 / 2,{ }^{7} / 2-585.8 \operatorname{sh}(17,070), 595.0(16,810), 606.5(16,490), 615.7$ (16,240), $646.2(15,480), 692.5(14,440), 704.5(14,190), 720.4(13,880), 739.2$ sh $(13,530)$, 782.7 (12,780), 799.1 (12,520), 822.9 (12,150), ${ }^{4} \mathrm{I}_{13 / 2}-881.3$ (11,350), 898.9 (11,120), 917.2 (10,900), $958.7(10,430),{ }^{4} \mathrm{~F}_{3 / 2}-1230.3(8,130),{ }^{4} \mathrm{I}_{11 / 2}-1649.7(6,060)$.

Crossover U/Zr experiment. In a manner similar to the synthesis of FLi(Be)M. LiF (38 mg, 1.5 $\mathrm{mmol}), \mathrm{BeO}(11 \mathrm{mg}, 0.44 \mathrm{mmol}), \mathrm{ZrO}_{2}(32 \mathrm{mg}, 0.26 \mathrm{mmol})$ and $\mathrm{UO}_{3} \cdot 2 \mathrm{H}_{2} \mathrm{O}(75 \mathrm{mg}, 0.23 \mathrm{mmol})$ 
were combined in a $12 \mathrm{~mL}$ Teflon cup and moved to a fume hood where $\mathrm{DI} \mathrm{H}_{2} \mathrm{O}(750 \mu \mathrm{L})$ and $\mathrm{HF}_{(\mathrm{aq})}(48$ wt $\%, 500 \mu \mathrm{L})$ was added. The cup was sealed, placed inside a steal jacket, removed from the fume hood, sealed and placed inside a box furnace. The heating profile consisted of a ramp from room temperature to $200{ }^{\circ} \mathrm{C}$ over $30 \mathrm{~min}$, a $72 \mathrm{~h}$ dwell followed by cooling to $25{ }^{\circ} \mathrm{C}$ at a rate of $4{ }^{\circ} \mathrm{C} / \mathrm{h}$. The jacket was unsealed in the fume hood and the Teflon cup was removed and carefully opened. The product was decanted, washed with $\mathrm{MeOH}(3 \times 1 \mathrm{~mL})$ and left to dry in air at least $3 \mathrm{~h}$ and X-ray quality crystals were selected upon drying. The reaction yielded green X-ray quality crystals identified to be LiUF 5 by crystallographic analysis. ${ }^{8-9}$

\section{UV/VIS/NIR SPECTROSCOPY AND PhOtograPhS OF CRYSTALS}

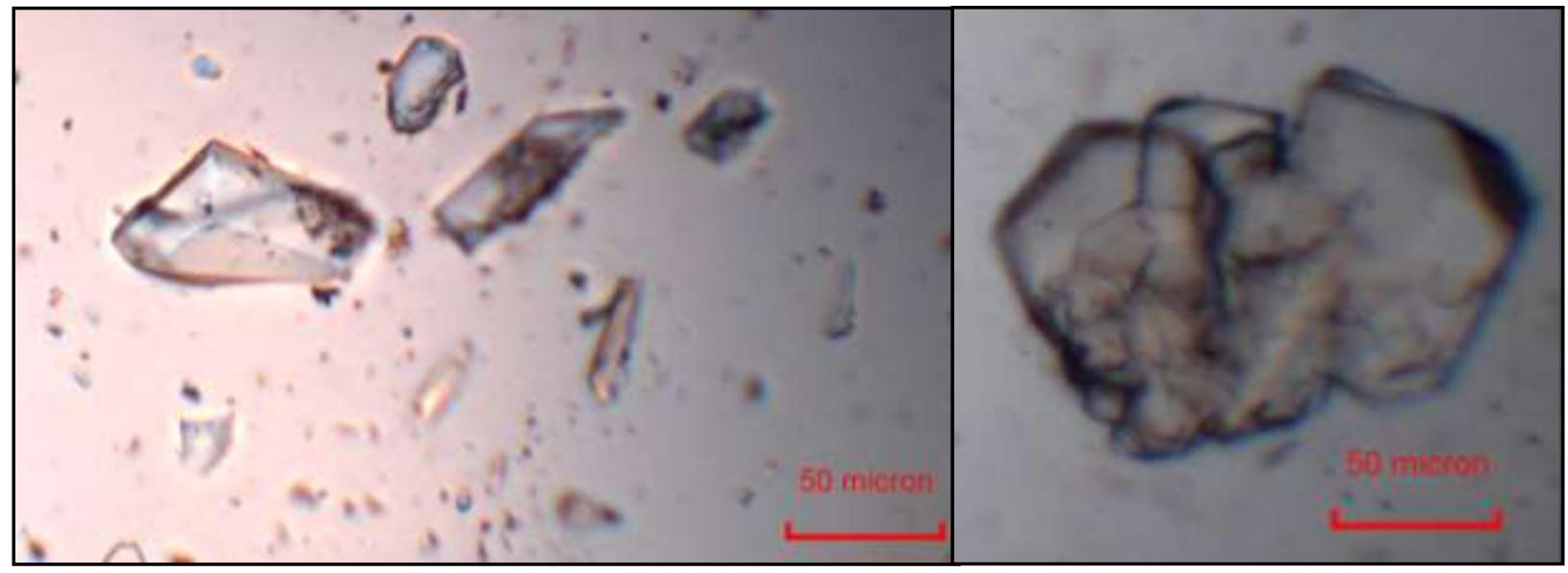

Figure S1. (Left) Photograph of $\mathrm{Li}_{4} \mathrm{CeF} 8$ crystals, (Right) photograph of $\mathrm{Ce}_{3} \mathrm{~F}_{12}\left(\mathrm{H}_{2} \mathrm{O}\right)$ crystals.

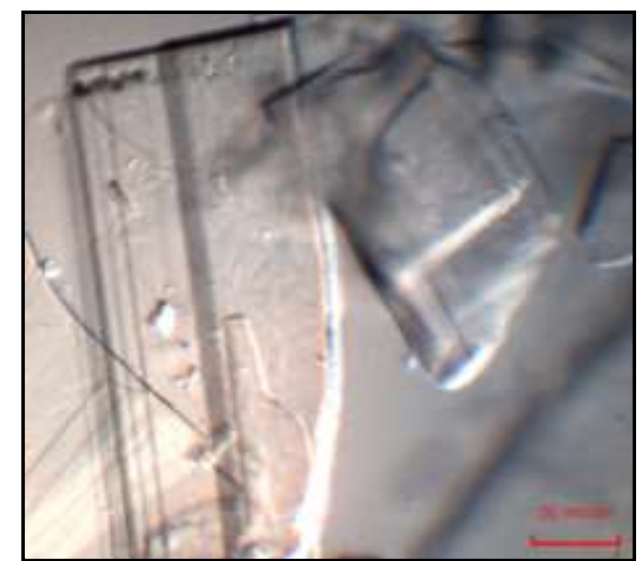

Figure S2. Photograph of $\mathrm{Li}_{6} \mathrm{BeF}_{4} \mathrm{ZrF}_{8}$ crystals. 


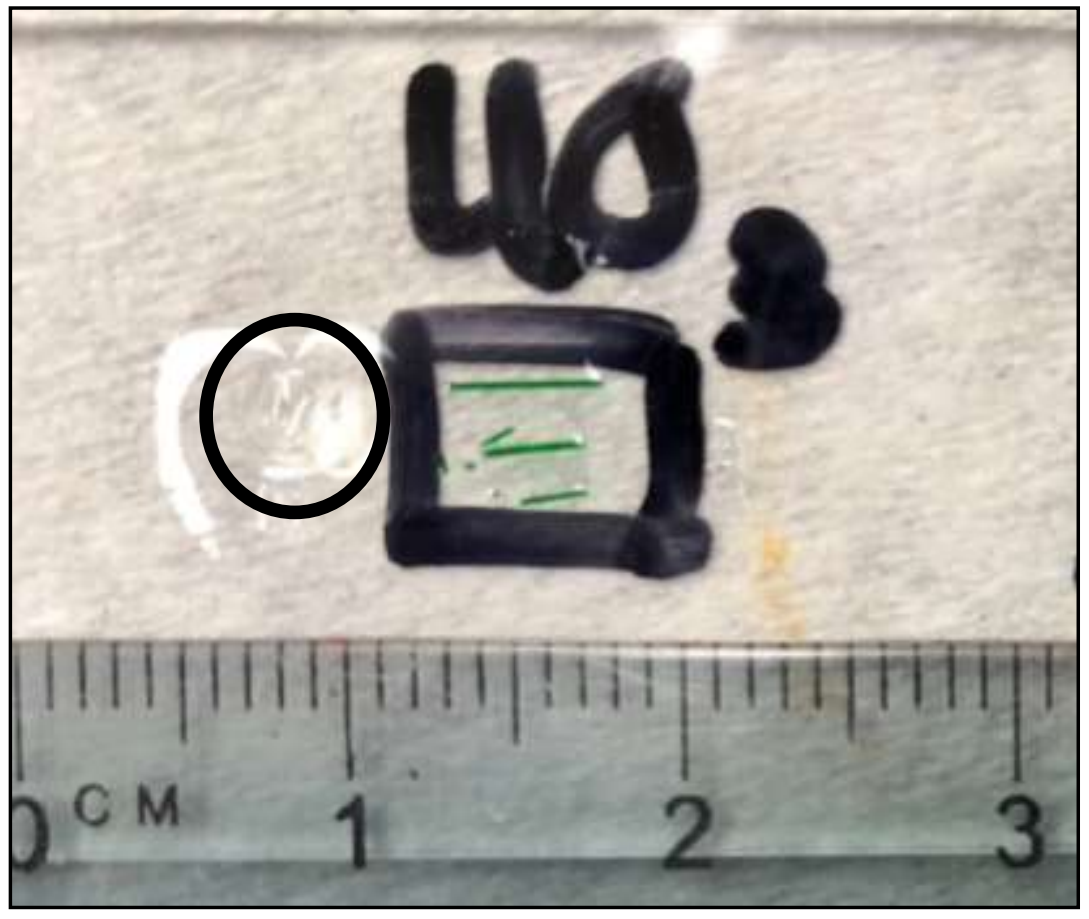

Figure S3. Photograph of $\mathbf{L i U F}_{5}$ crystals grown from $\mathrm{UO}_{3} \cdot 2 \mathrm{H}_{2} \mathrm{O}$ with a centimeter ruler for scale, a large crystal of $\mathbf{L i}_{2} \mathbf{B e F}_{4}$ is circled to the left, see below.

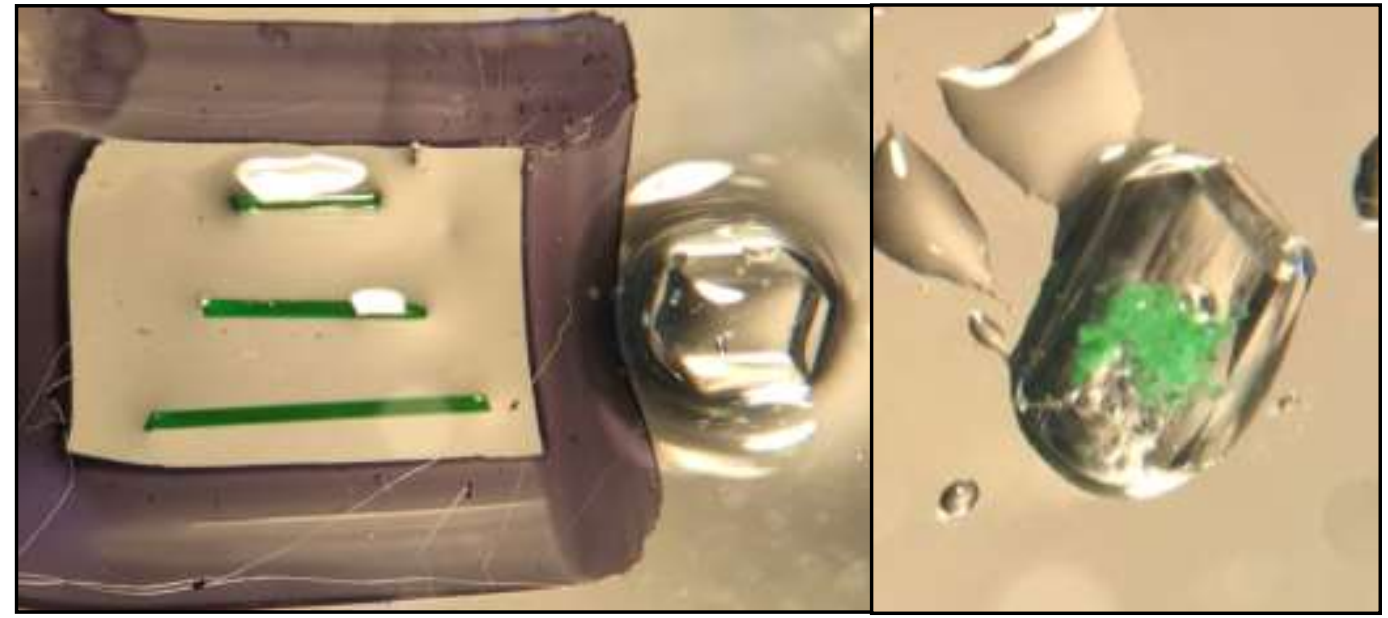

Figure S4. (Left) Photograph of LiUF5 crystals grow from $\mathrm{UO}_{3} \cdot 2 \mathrm{H}_{2} \mathrm{O}$ with a large crystal of $\mathbf{L i}_{2} \mathbf{B e F}_{4}$ crystal, taken through a $4 \mathrm{X}$ zoom microscope. (Right) Photograph of LiUF5 material encased within $\mathbf{L i}_{2} \mathbf{B e F}_{4}$ matrix taken through a $4 \mathrm{X}$ zoom microscope. 


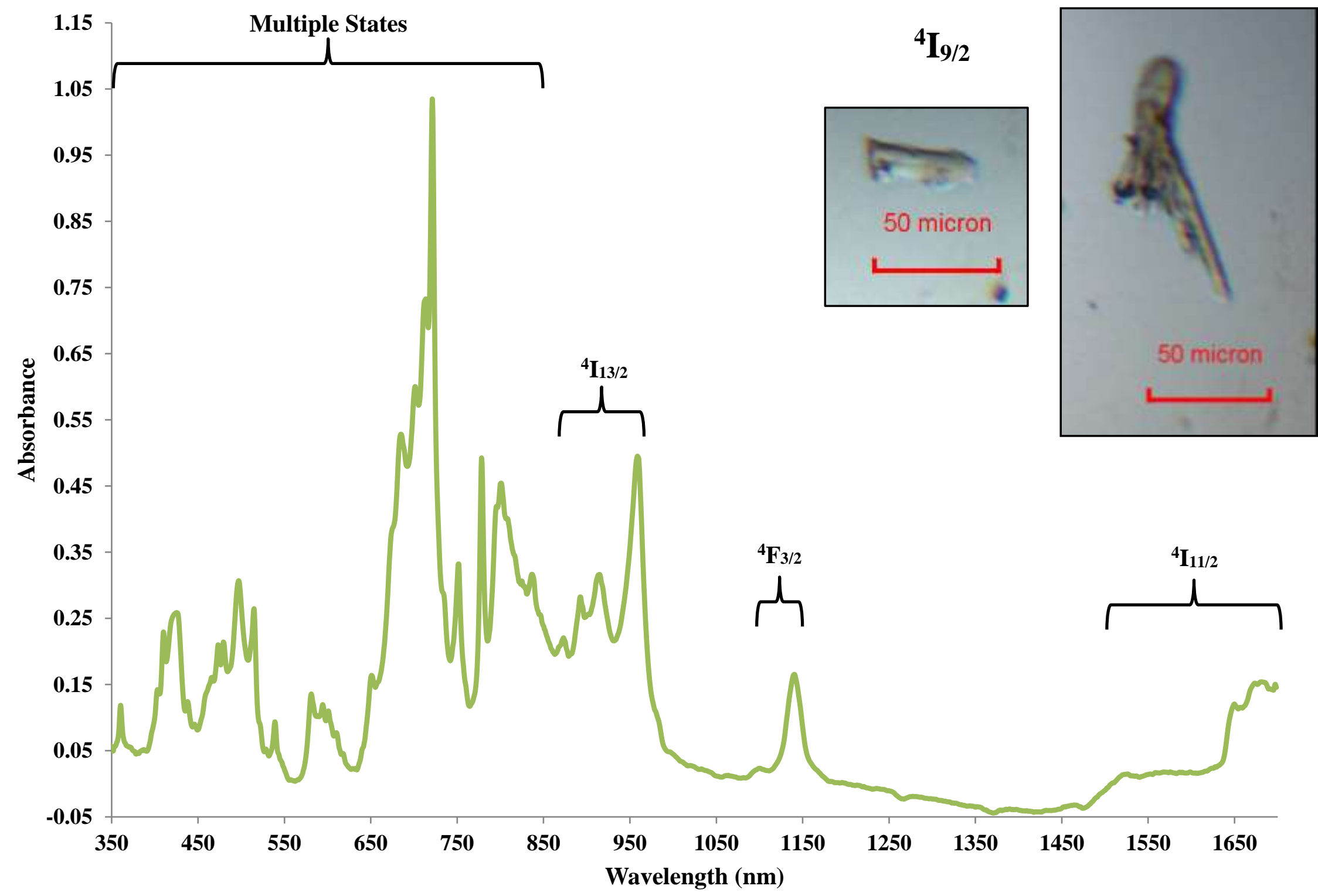

Figure S5. Room temperature solid state UV/vis/NIR spectrum of $\mathbf{L i N p F}$, with known excited states labeled based on $\mathrm{NpF}_{4}{ }^{19}$ and photographs of crystals in upper right corner. 


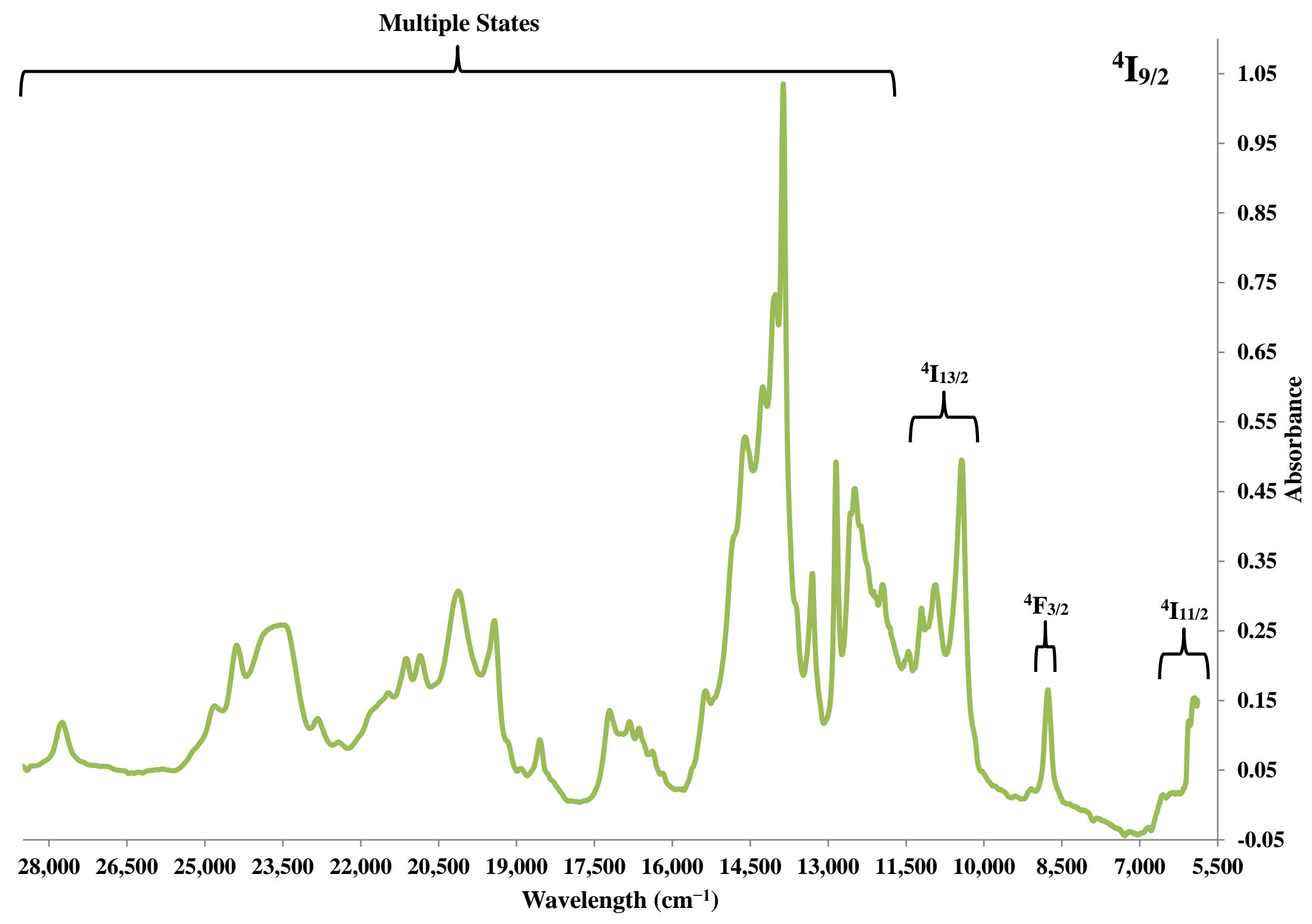

Figure S6. Room temperature solid state UV/vis/NIR spectrum of LiNpF5 plotted in energy with known excited states labeled based on $\mathrm{NpF}_{4}{ }^{19}$ 


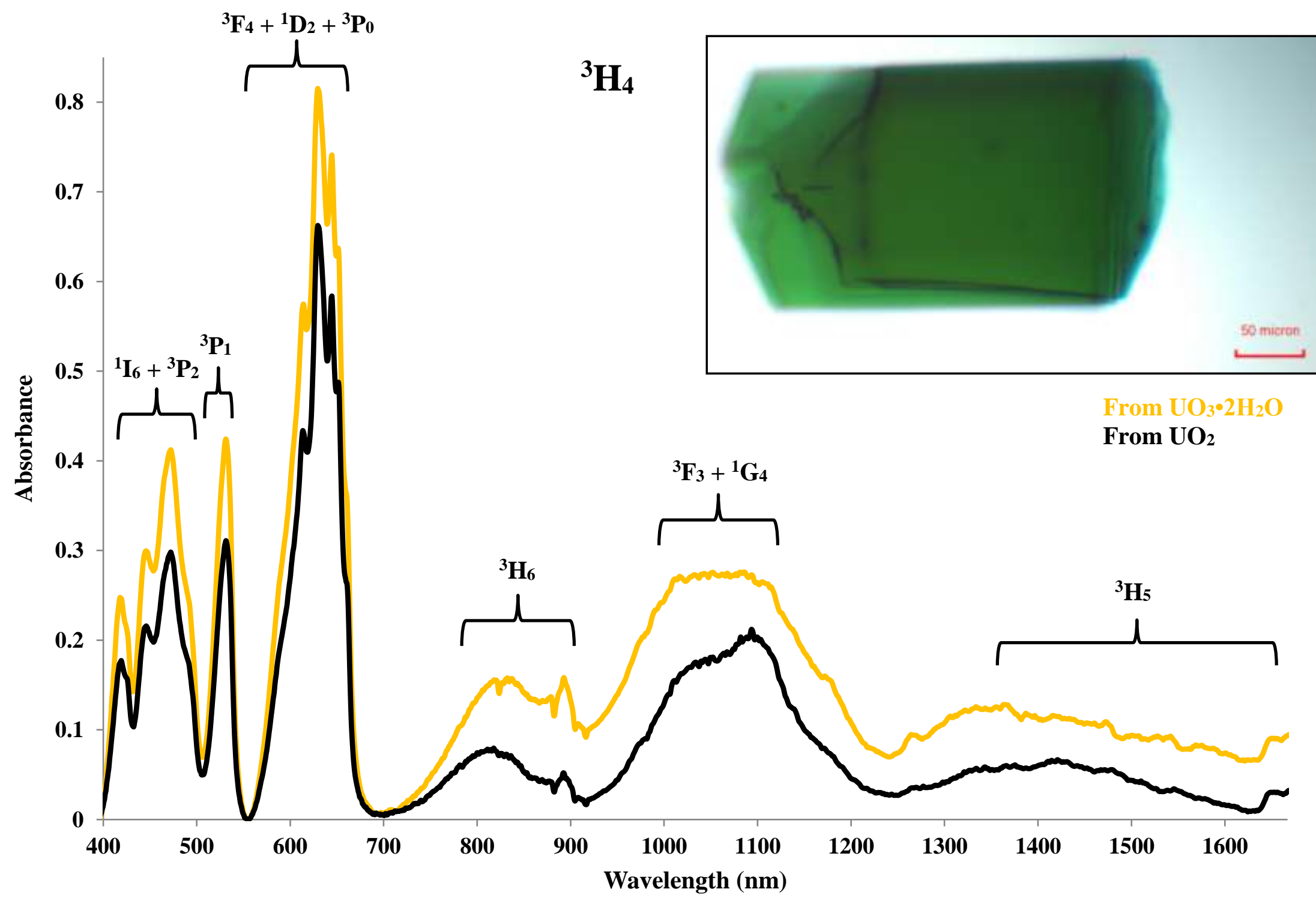

Figure S7. Room temperature solid state UV/vis/NIR spectra of $\mathbf{L i U F} 5$ as synthesized from $\mathrm{UO}_{2}$ (black trace) and $\mathrm{UO}_{3} \cdot 2 \mathrm{H}_{2} \mathrm{O}($ orange trace) with excited states labeled based on $\mathrm{UF}_{4},{ }^{19}$ and photograph of LiUF5 crystal synthesized using $\mathrm{UO}_{3} \cdot 2 \mathrm{H}_{2} \mathrm{O}$ in upper right hand corner. 


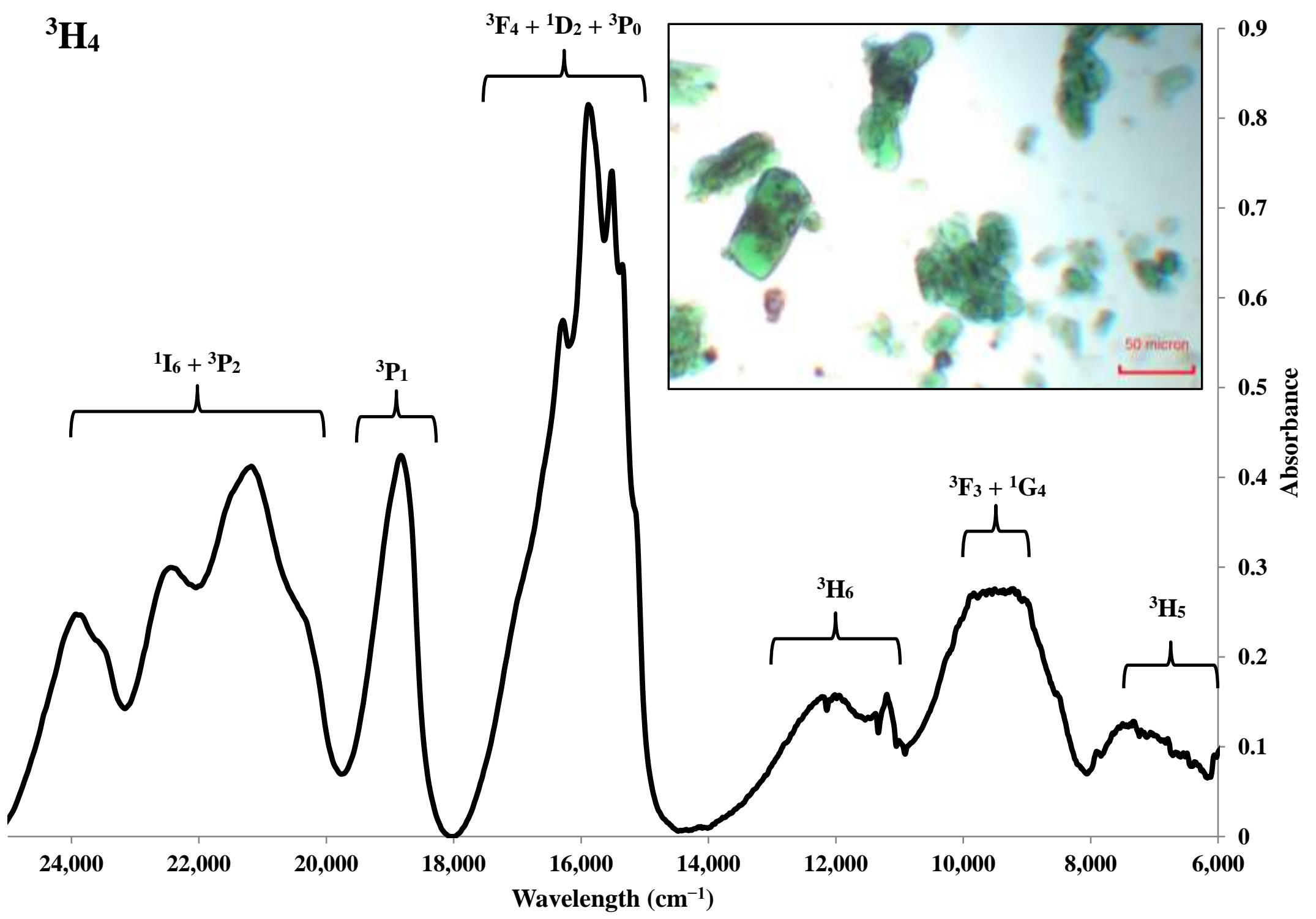

Figure S8. Room temperature solid state UV/vis/NIR spectra of LiUF5 as synthesized from $\mathrm{UO}_{3} \cdot 2 \mathrm{H}_{2} \mathrm{O}$ plotted in energy with excited states labeled based on $\mathrm{UF}_{4},{ }^{19}$ and photograph of $\mathbf{L i U F} 5$ crystals synthesized using $\mathrm{UO}_{2}$ in upper right hand corner. 


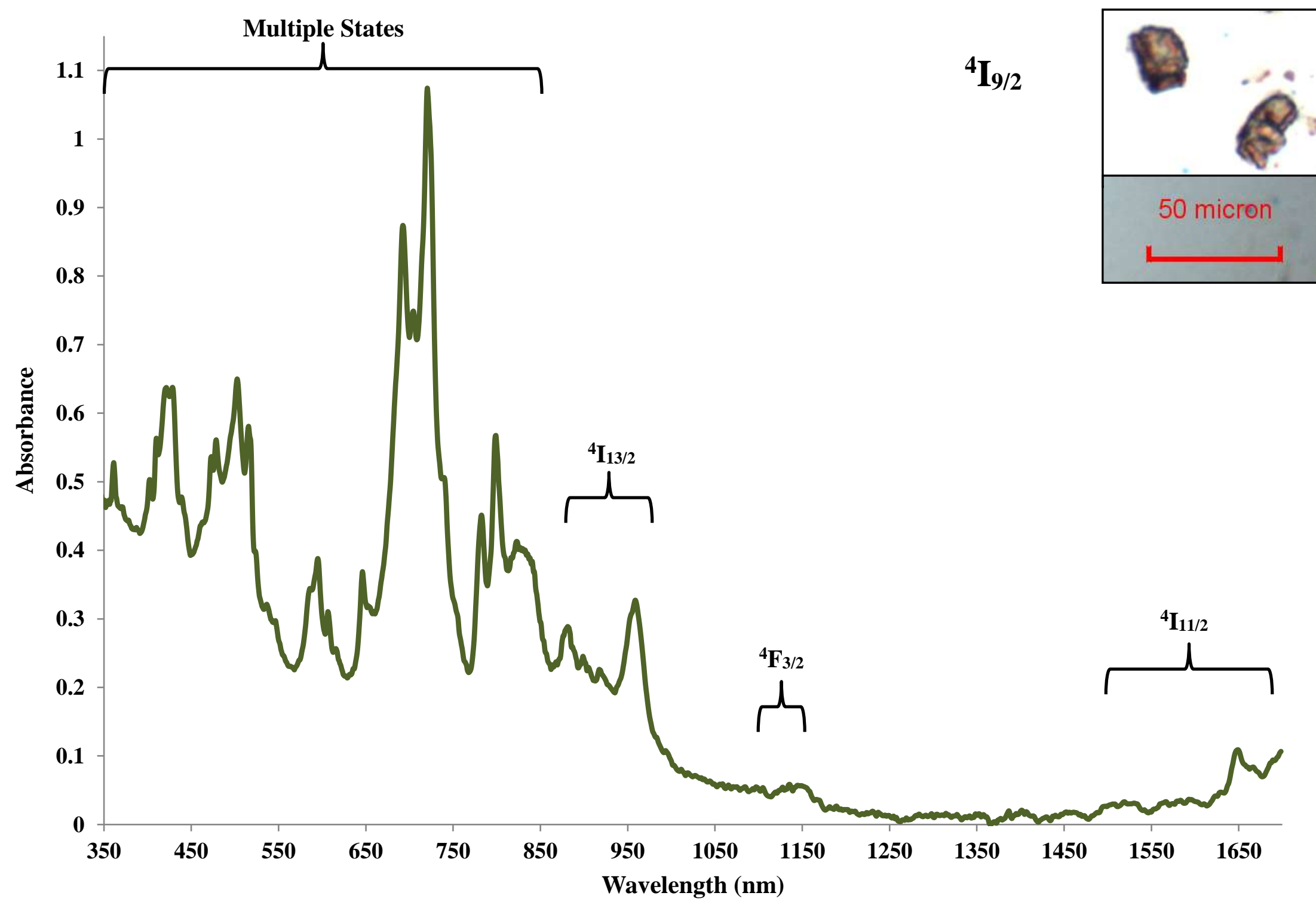

Figure S9. Room temperature solid state UV/vis/NIR spectra of $\mathbf{N p}_{3} \mathbf{F}_{12}\left(\mathbf{H}_{2} \mathbf{O}\right)$ with excited states labeled based on $\mathrm{NpF}_{4}$, ${ }^{19}$ and photograph crystals in upper right hand corner. 


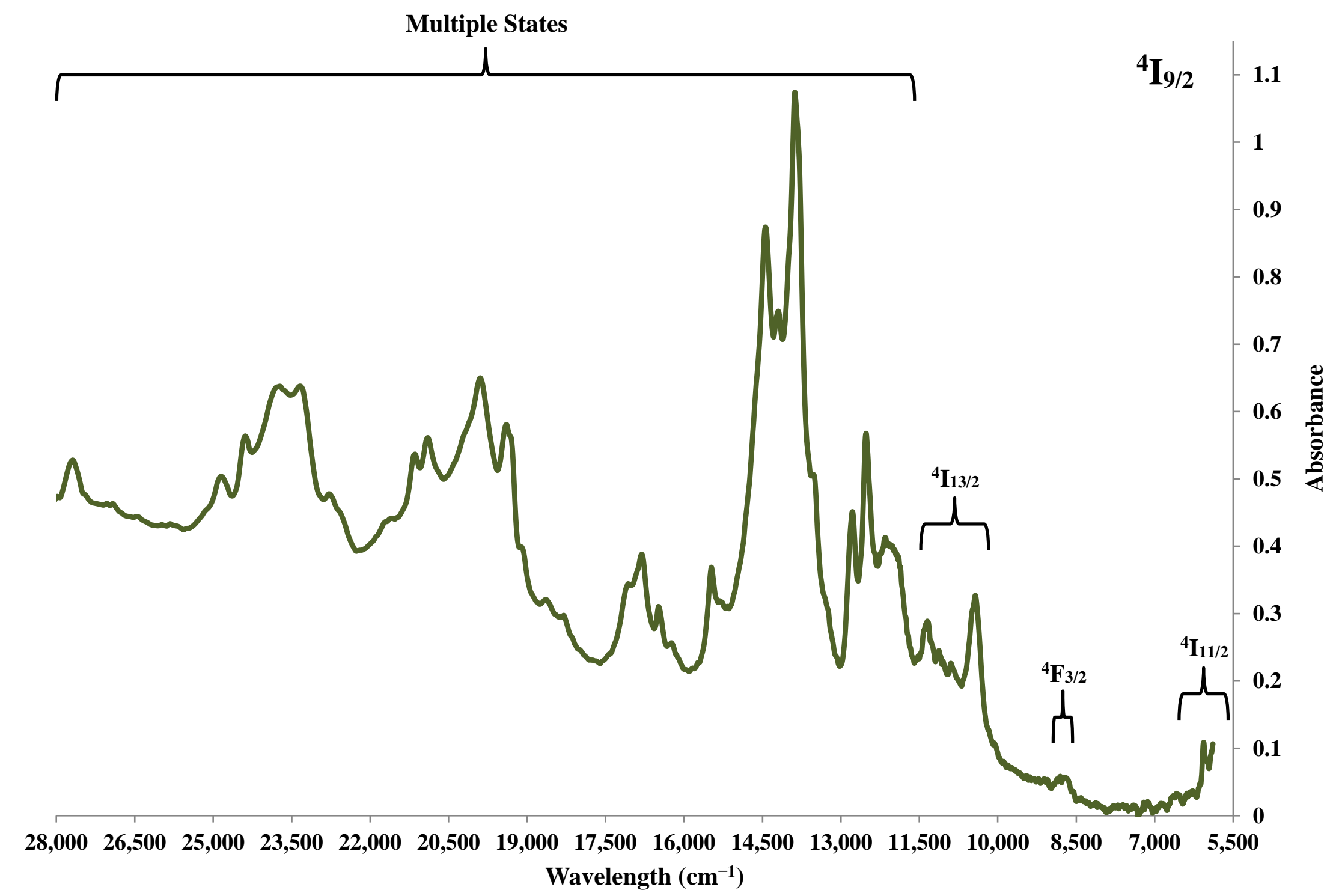

Figure S10. Room temperature solid state UV/vis/NIR spectra of $\mathrm{Np}_{3} \mathbf{F}_{12}\left(\mathrm{H}_{2} \mathrm{O}\right)$ plotted in energy with excited states labeled based on $\mathrm{NpF}_{4}{ }^{19}$ 


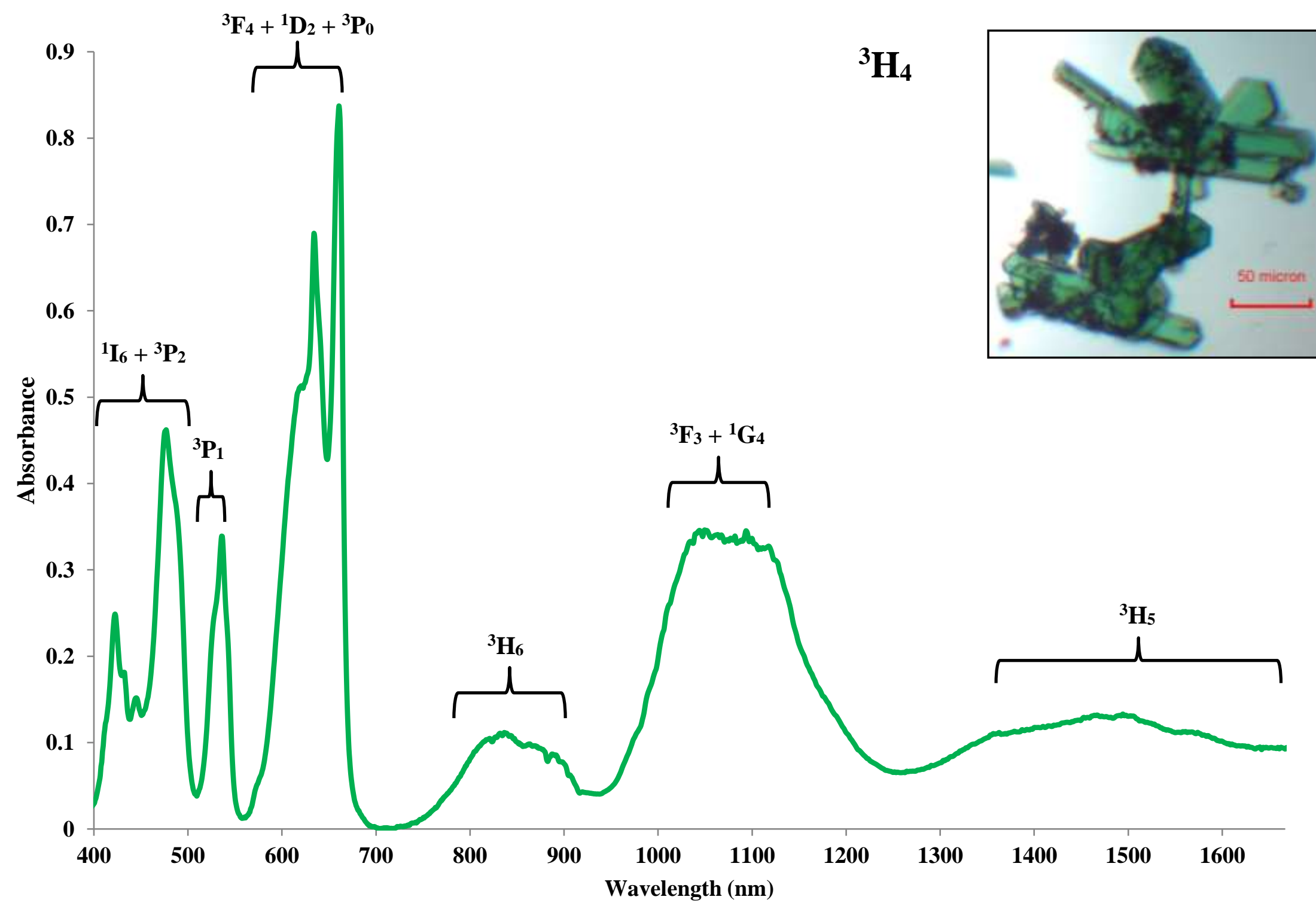

Figure S11. Room temperature solid state UV/vis/NIR spectra of $\mathbf{U}_{3} \mathbf{F}_{12}\left(\mathbf{H}_{2} \mathbf{O}\right)$ with excited states labeled based on $\mathrm{UF}_{4}$, ${ }^{19}$ and photograph crystals in upper right hand corner. 


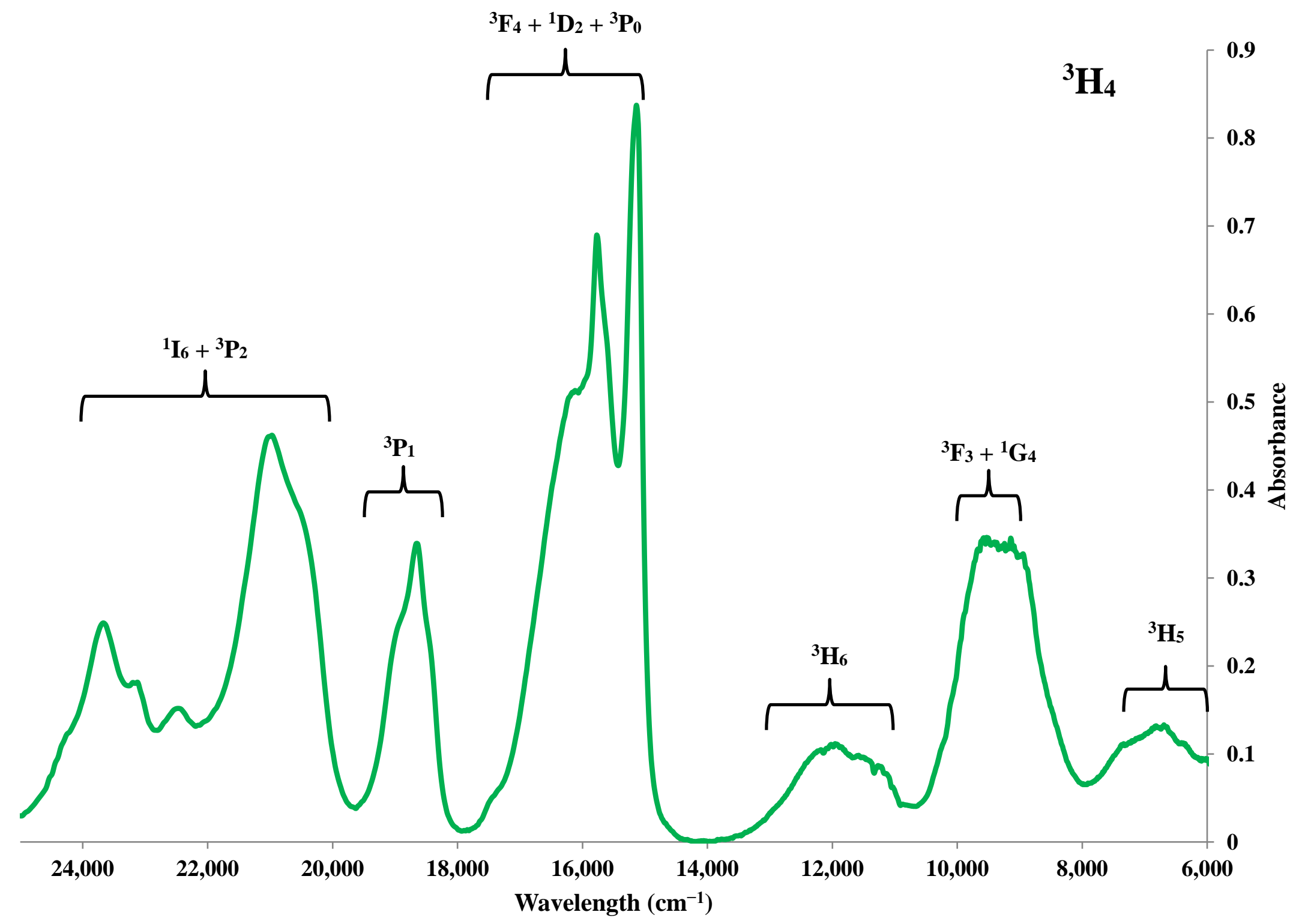

Figure S12. Room temperature solid state UV/vis/NIR spectra of $\mathbf{U}_{\mathbf{3}} \mathbf{F}_{\mathbf{1 2}}\left(\mathbf{H}_{2} \mathbf{O}\right)$ plotted in energy with excited states labeled based on $\mathrm{UF}_{4 . .}{ }^{19}$ 


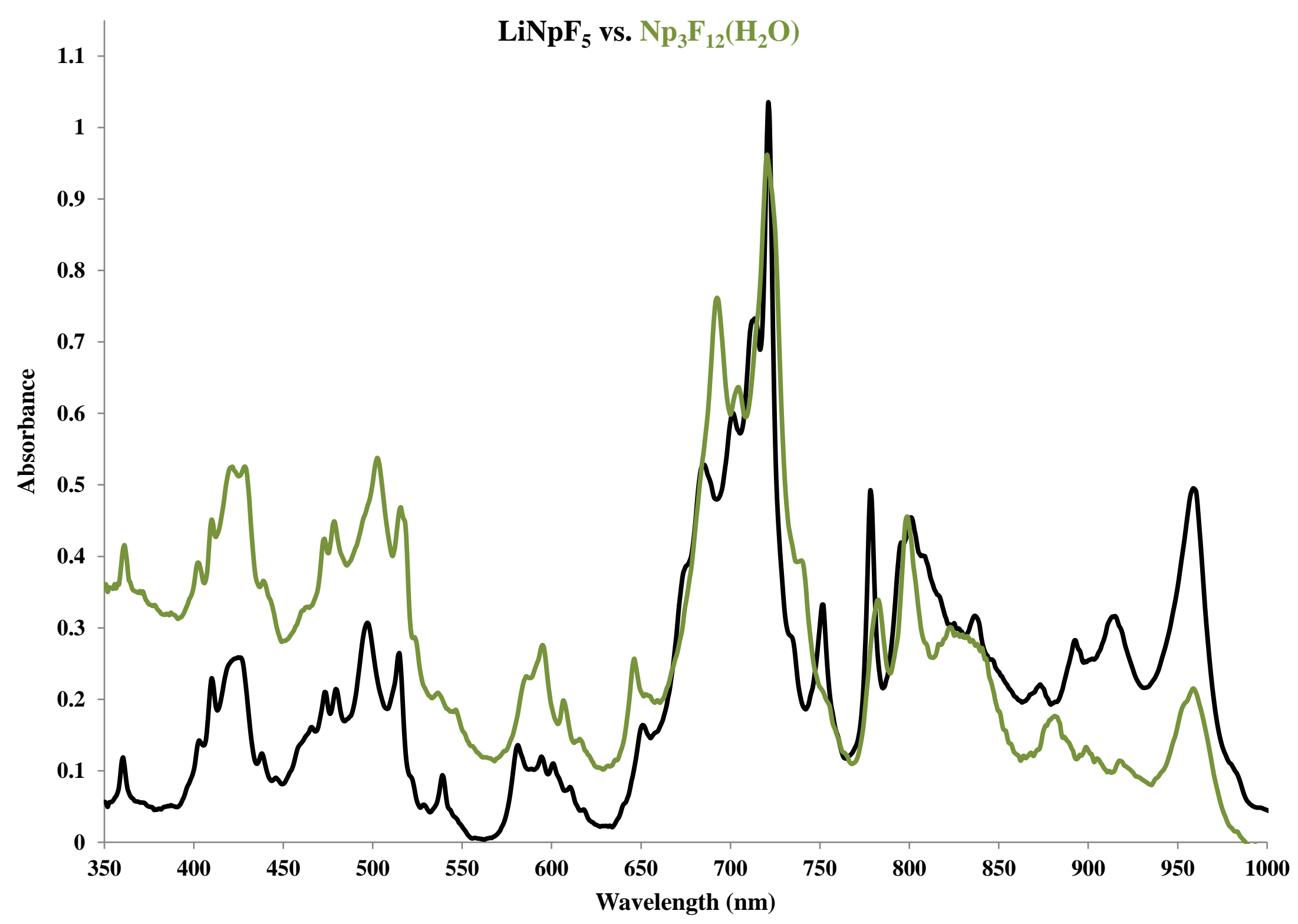

Figure S13. Comparison of UV/vis/NIR spectra of $\mathbf{L i N p F} 5$ (black trace) and $\mathbf{N p}_{3} \mathbf{F}_{12}\left(\mathbf{H}_{2} \mathrm{O}\right.$ ) (green trace). 


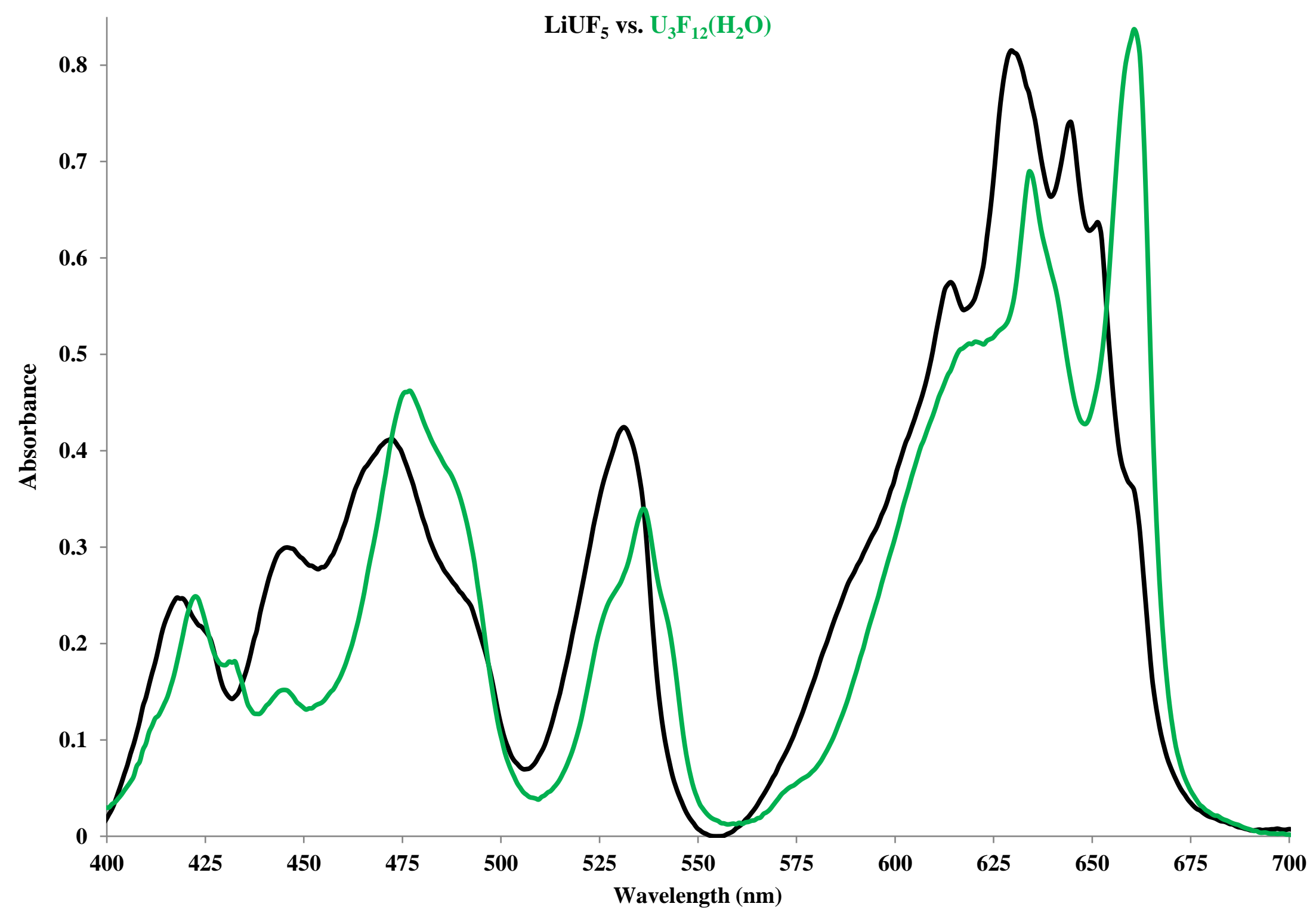

Figure S14. Comparison of UV/vis/NIR spectra of LiUF5 (black trace) and $\mathbf{U}_{3} \mathbf{F}_{12}\left(\mathrm{H}_{2} \mathrm{O}\right)$ (green trace). 


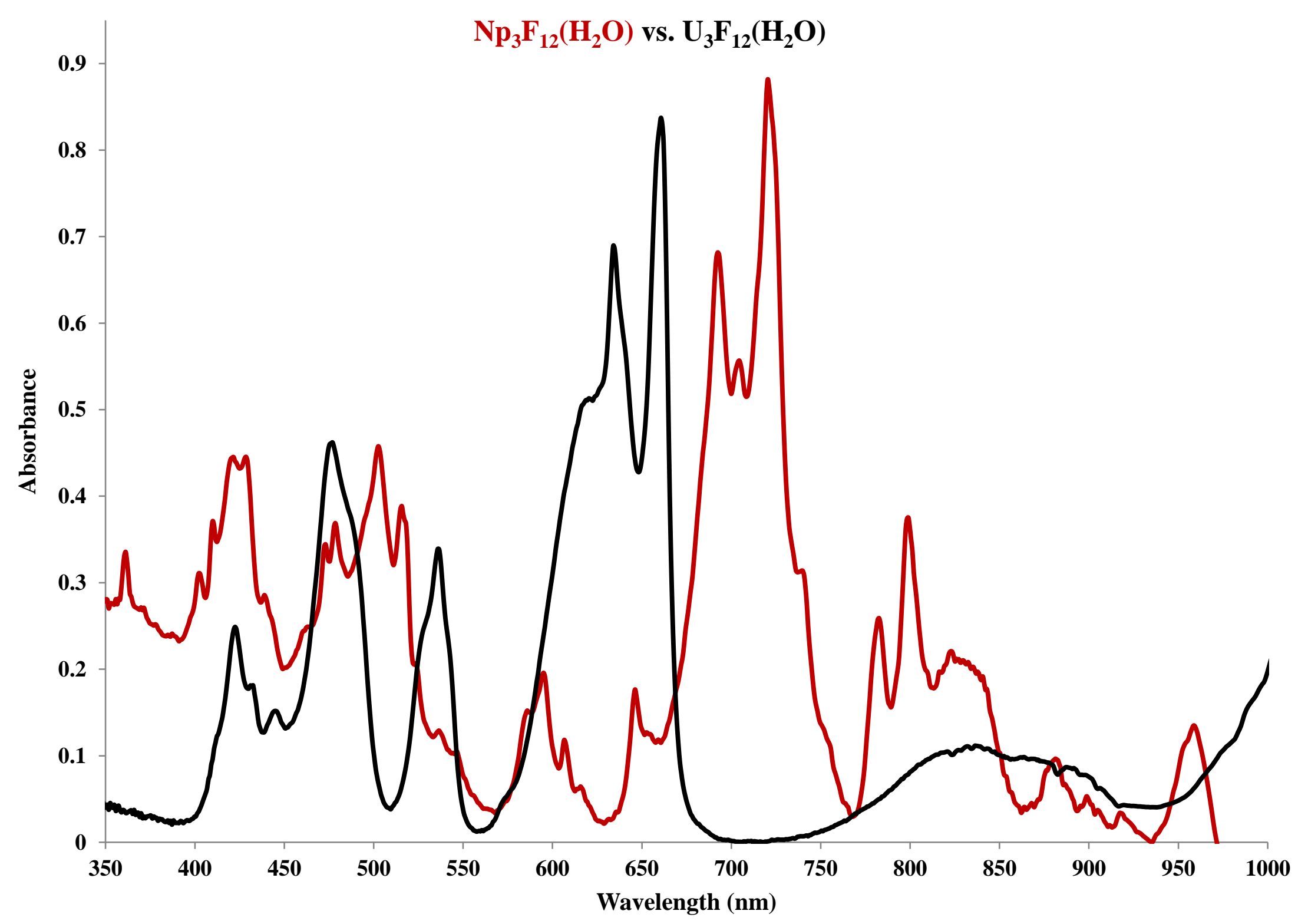

Figure S15. Comparison of UV/vis/NIR spectra of $\mathbf{A n}_{3} \mathbf{F}_{12}\left(\mathbf{H}_{2} \mathbf{O}\right)(A n=N p$, red trace; U black trace). 


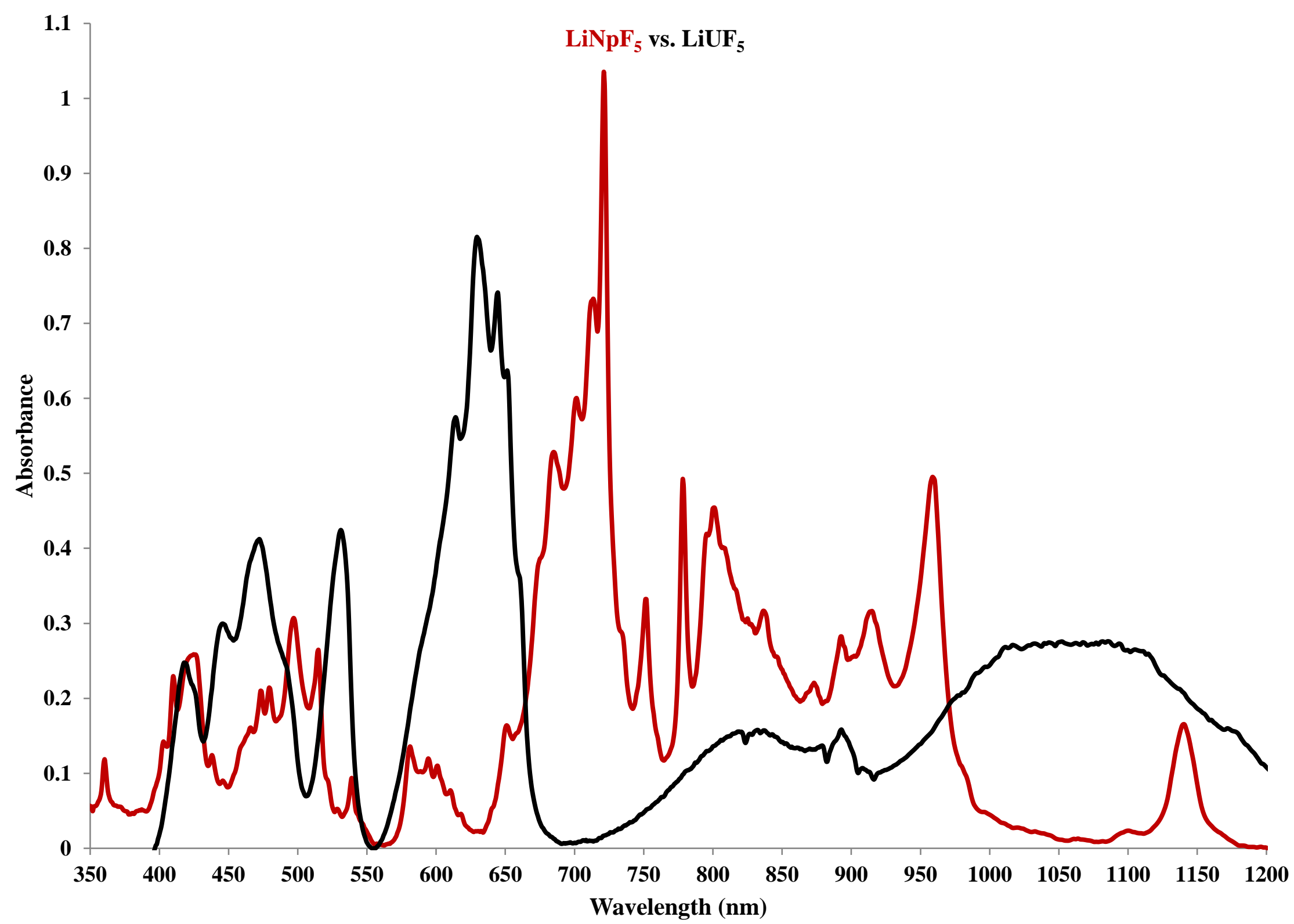

Figure S16. Comparison of UV/vis/NIR spectra of $\mathbf{L i A n F}_{5}(\mathrm{An}=\mathrm{Np}$, red trace; U black trace). 


\section{BOND COMPARISONS}

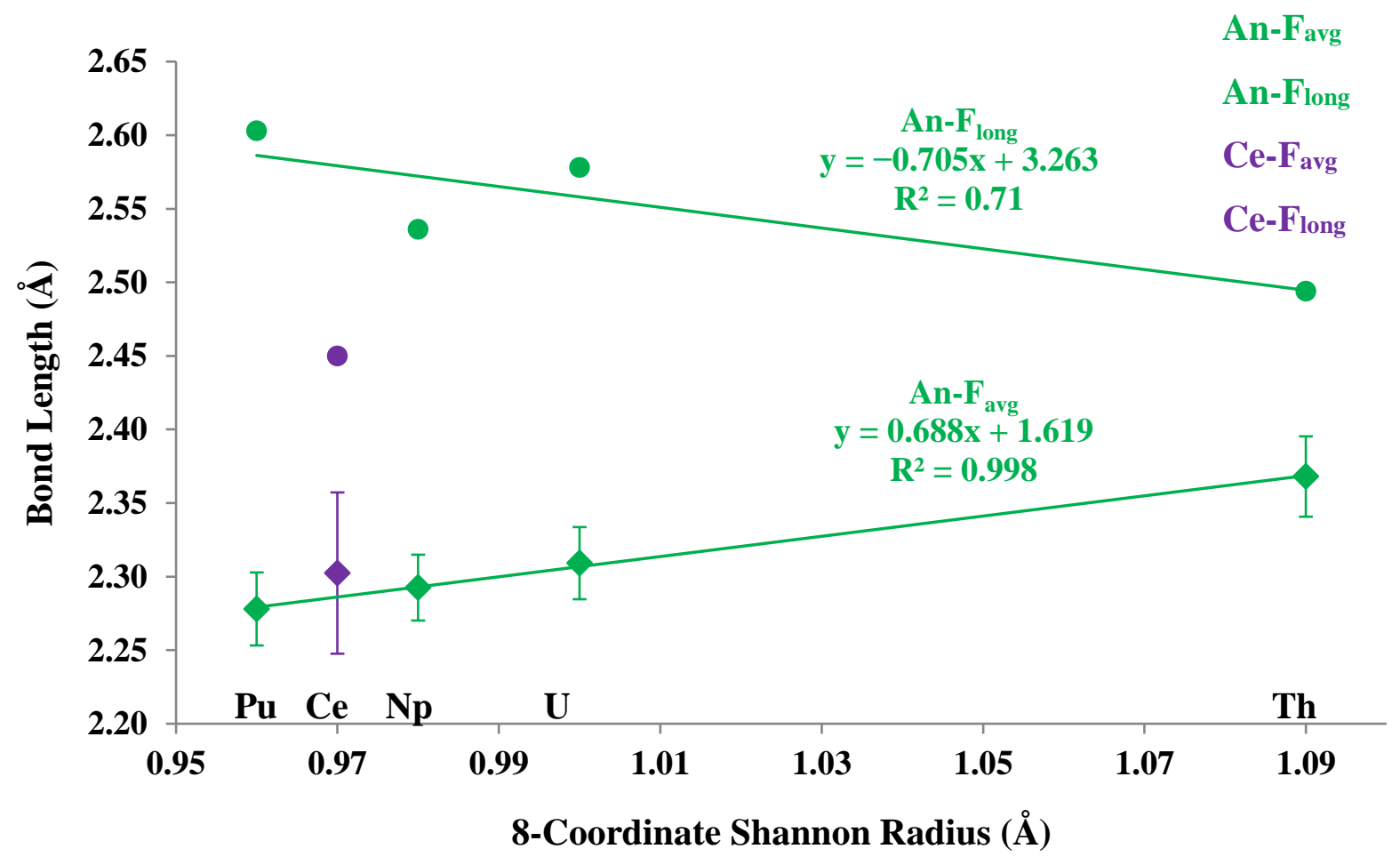

Figure S17. Plot of bond distances in LiMF5 with $\mathrm{An}-\mathrm{F}(\mathrm{avg})\left(\mathrm{An}=\mathrm{Pu},{ }^{26} \mathrm{~Np}, \mathrm{U},{ }^{9} \mathrm{Th} ;{ }^{27}\right.$ green diamonds), $\mathrm{Ce}-\mathrm{F}^{27}$ (purple diamond) plotted with standard error and longest An-F bond (green circles) and longest $\mathrm{Ce}-\mathrm{F}$ bond (purple circle)

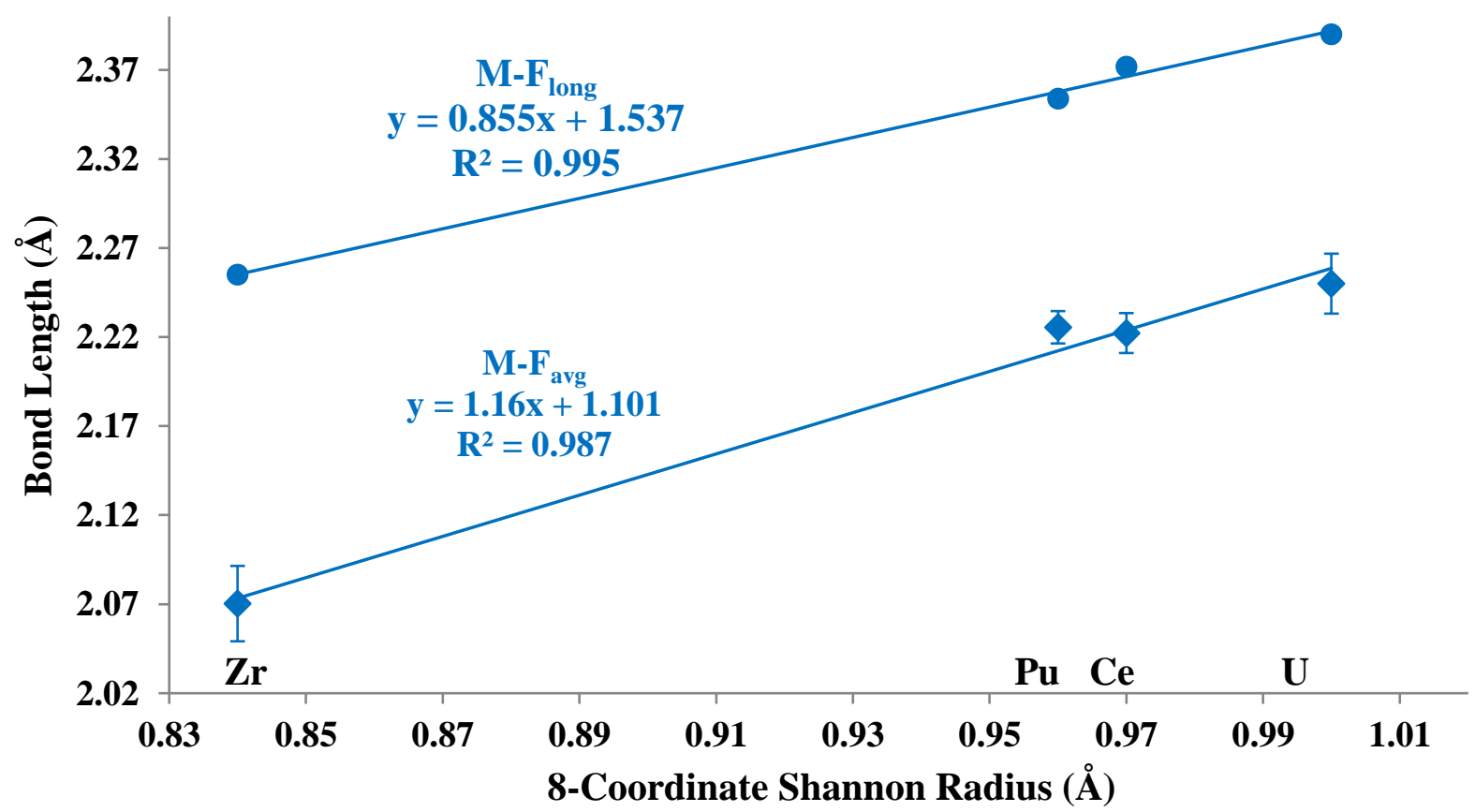

Figure S18. Plot of bond distances in single crystal collections of $\mathbf{L i 4 M F 8}\left(\mathrm{M}=\mathrm{Zr},{ }^{28} \mathrm{Pu},{ }^{26} \mathrm{Ce}\right.$, $\mathrm{U}^{10}$ ) with average $\mathrm{M}-\mathrm{F}$ bonds (blue diamonds) plotted with standard error and longest $\mathrm{M}-\mathrm{F}$ bonds (blue circles). 


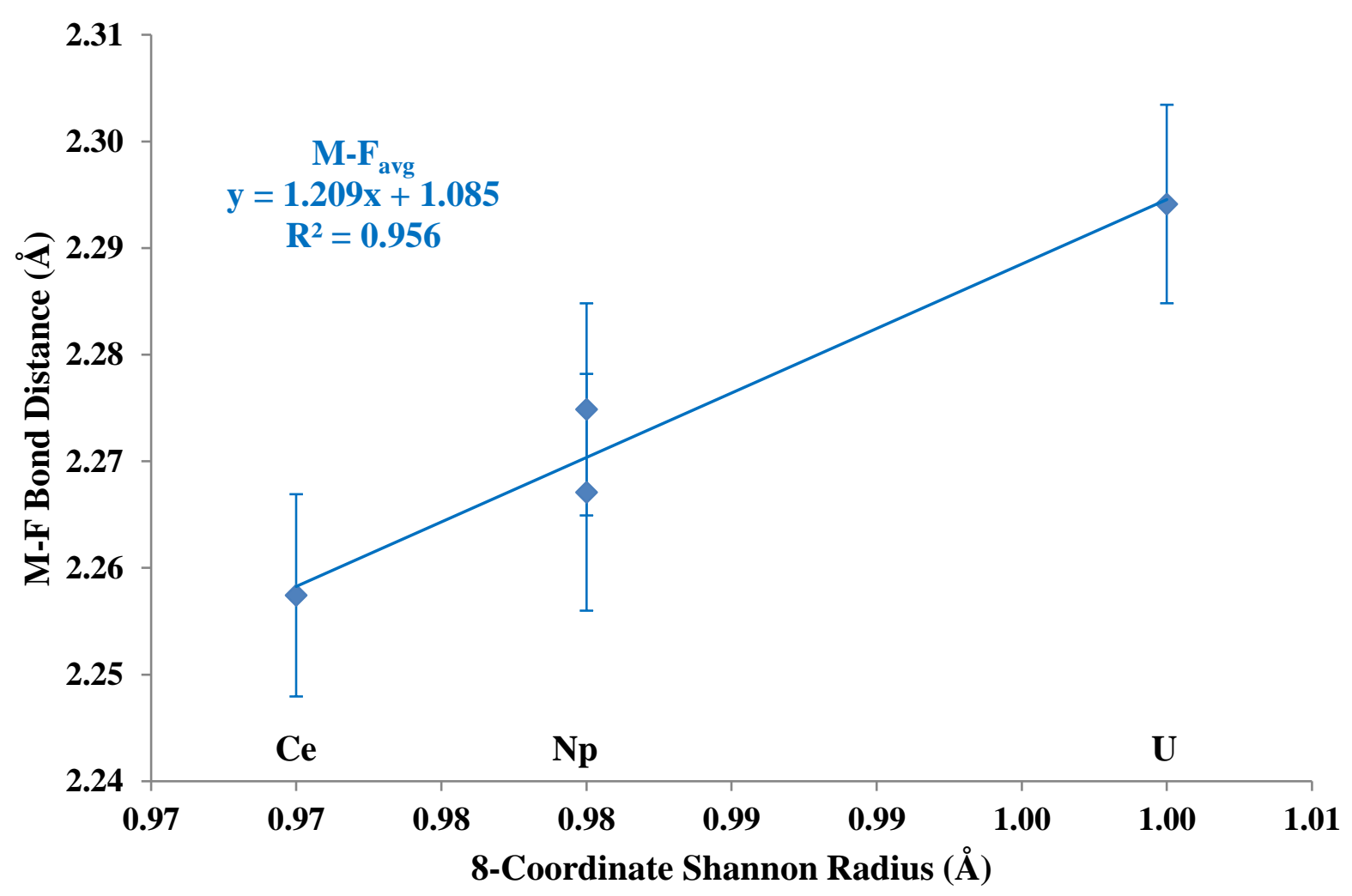

Figure S19. Plot of bond distances in single crystal collections of $\mathbf{M}_{3} \mathbf{F}_{12}\left(\mathrm{H}_{2} \mathbf{O}\right)\left(\mathrm{M}=\mathrm{Ce}, \mathrm{Np},{ }^{29}\right.$ $\mathrm{U}^{14}$ ) with average $\mathrm{M}-\mathrm{F}$ bonds (blue diamonds) plotted with standard error.

\section{Crystallographic Details}

Table S1. Summary of Crystallographic Collections for $\mathrm{LiNpF}_{5}, \mathrm{Li}_{4} \mathrm{CeF}_{8}, \mathrm{Li}_{6} \mathrm{BeF}_{4} \mathrm{ZrF}_{8}$, $\mathrm{Np}_{3} \mathrm{~F}_{12}\left(\mathrm{H}_{2} \mathrm{O}\right)$ and $\mathrm{Ce}_{3} \mathrm{~F}_{12}\left(\mathrm{H}_{2} \mathrm{O}\right)$.

\begin{tabular}{|c|c|c|c|c|c|}
\hline Compound & LiNpF $_{5}$ & $\mathrm{Li}_{4} \mathrm{CeF}_{8}$ & $\mathrm{Li}_{6} \mathrm{BeF}_{4} \mathrm{ZrF}_{8}$ & $\mathrm{~Np}_{3} \mathrm{~F}_{12}\left(\mathrm{H}_{2} \mathrm{O}\right)$ & $\mathrm{Ce}_{3} \mathrm{~F}_{12}\left(\mathrm{H}_{2} \mathrm{O}\right)$ \\
\hline Identifier & cjw100 & cjw71 & cjw72 & cjw102 & cjw111 \\
\hline $\begin{array}{l}\text { Empirical } \\
\text { Formula }\end{array}$ & $\mathrm{Li}_{8} \mathrm{~F}_{40} \mathrm{~Np}_{8}$ & $\mathrm{Li}_{4} \mathrm{~F}_{8} \mathrm{Ce}$ & $\mathrm{Li}_{12} \mathrm{Be}_{2} \mathrm{~F}_{24} \mathrm{Zr}_{2}$ & $\mathrm{OH}_{2} \mathrm{~F}_{12} \mathrm{~Np}_{3}$ & $\mathrm{OH}_{2} \mathrm{~F}_{12} \mathrm{Ce}_{3}$ \\
\hline Formula Weight & 2711.52 & 319.88 & 739.74 & 957.02 & 666.38 \\
\hline Color & Green & Colorless & Colorless & Green & Colorless \\
\hline Size (mm) & $\begin{array}{c}0.551 \times 0.074 \\
\times 0.049\end{array}$ & $\begin{array}{c}0.282 \times 0.120 \\
\times 0.094\end{array}$ & $\begin{array}{c}0.28 \times 0.24 \times \\
0.18\end{array}$ & $\begin{array}{c}0.008 \times 0.005 \\
\times 0.003\end{array}$ & $\begin{array}{l}0.242 \times 0.164 \\
\quad \times 0.090\end{array}$ \\
\hline Radiation $(\lambda, \AA)$ & Mo (0.71073) & Mo (0.71073) & Mo (0.71073) & Mo (0.71073) & Mo (0.71073) \\
\hline Temp (K) & $100(2)$ & $120(2)$ & $120(2)$ & $100(2)$ & $100(2)$ \\
\hline
\end{tabular}




\begin{tabular}{|c|c|c|c|c|c|}
\hline Crystal System & Tetragonal & Orthorhombic & Tetragonal & Monoclinic & Monoclinic \\
\hline Space Group & $I 41 / a$ & Pnma & I4 $1 /$ amd & Im & Im \\
\hline$a(\AA)$ & $14.7242(6)$ & 9.933(2) & $6.587(2)$ & $8.453(1)$ & $8.4092(4)$ \\
\hline$b(\AA)$ & $14.7242(6)$ & $9.818(2)$ & $6.587(2)$ & $11.736(2)$ & $11.6635(6)$ \\
\hline$c(\AA)$ & $6.4647(3)$ & $5.922(1)$ & $18.565(6)$ & $9.437(2)$ & $9.3604(7)$ \\
\hline$\beta\left(^{\circ}\right)$ & 90 & 90 & 90 & $95.546(2)$ & $95.607(1)$ \\
\hline Volume $\left(\AA^{3}\right)$ & 1401.6(1) & $577.5(2)$ & $805.5(6)$ & $931.8(3)$ & 913.7(1) \\
\hline $\mathbf{Z}$ & 2 & 4 & 2 & 4 & 4 \\
\hline$\rho_{\text {calcd }}\left(\mathrm{Mg} / \mathbf{m}^{3}\right)$ & 6.425 & 3.679 & 3.050 & 6.822 & 4.844 \\
\hline$\mu\left(\mathrm{mm}^{-1}\right)$ & 29.635 & 7.980 & 1.542 & 33.376 & 14.864 \\
\hline$F(000)$ & 2256 & 568 & 680 & 1588 & 1168 \\
\hline$\Theta$ range $\left({ }^{\circ}\right)$ & $2.767-27.448$ & $4.006-30.549$ & $3.282-30.502$ & $2.778-25.349$ & $2.798-30.528$ \\
\hline Index Range & $\begin{array}{c}-19 \leq \mathrm{h} \leq 19 \\
-19 \leq \mathrm{k} \leq 19 \\
-8 \leq 1 \leq 8\end{array}$ & $\begin{array}{c}-14 \leq \mathrm{h} \leq 14 \\
-14 \leq \mathrm{k} \leq 14 \\
-8 \leq 1 \leq 8\end{array}$ & $\begin{aligned}-9 & \leq \mathrm{h} \leq 9 \\
-9 & \leq \mathrm{k} \leq 9 \\
-26 & \leq 1 \leq 26\end{aligned}$ & $\begin{array}{c}-10 \leq \mathrm{h} \leq 10 \\
-14 \leq \mathrm{k} \leq 14 \\
-11 \leq 1 \leq 11\end{array}$ & $\begin{array}{c}-12 \leq \mathrm{h} \leq 12 \\
-16 \leq \mathrm{k} \leq 16, \\
-13 \leq 1 \leq 13\end{array}$ \\
\hline Refl. Collected & 10149 & 10361 & 6782 & 4128 & 13312 \\
\hline $\begin{array}{c}\text { Indept. Refl. } \\
\text { [R(int)] }\end{array}$ & 802 [0.0265] & 924 [0.0402] & 360 [0.0543] & 1765 [0.0389] & 2900 [0.0246] \\
\hline $\begin{array}{c}\text { Completeness } \\
(\%)\end{array}$ & 99.8 & 98.6 & 99.5 & 100.0 & 99.3 \\
\hline Max/Min Trans. & $0.7461 / 0.5206$ & $0.7461 / 0.5331$ & $0.7461 / 0.5725$ & $0.0206 / 0.0049$ & $0.7461 / 0.5550$ \\
\hline $\begin{array}{c}\text { Data / Restraints } \\
\text { / Parameters }\end{array}$ & $802 / 0 / 64$ & 924 / 0 / 68 & $360 / 0 / 33$ & $1765 / 20 / 158$ & $2900 / 2 / 167$ \\
\hline GOOF & 1.129 & 1.226 & 1.112 & 1.129 & 1.117 \\
\hline $\mathrm{R1}^{a}(I>2.0 \sigma(\mathrm{I}))$ & 0.0121 & 0.0397 & 0.0159 & 0.0388 & 0.0116 \\
\hline wR2 (all data) & 0.0273 & 0.1074 & 0.0419 & 0.0861 & 0.0244 \\
\hline $\begin{array}{c}\text { Largest } \\
\text { peak/hole }\left(\mathrm{e}^{-} \AA^{-3}\right)\end{array}$ & $1.225 /-0.745$ & $4.432 /-1.899$ & $0.384 /-0.751$ & $2.602 /-1.663$ & $0.538 /-0.538$ \\
\hline BASF & & & & $0.4(1)$ & $0.22(2)$ \\
\hline
\end{tabular}

a Definitions: $\mathrm{wR} 2=\left\{\Sigma\left[\mathrm{w}\left(\mathrm{F}_{\mathrm{o}}{ }^{2}-\mathrm{F}_{\mathrm{c}}{ }^{2}\right)^{2}\right] / \Sigma\left[\mathrm{w}\left(\mathrm{F}_{\mathrm{o}}{ }^{2}\right)^{2}\right]\right\}^{1 / 2} \mathrm{R} 1=\Sigma|| \mathrm{F}_{\mathrm{o}}|-| \mathrm{F}_{\mathrm{c}}|/ \Sigma| \mathrm{F}_{\mathrm{o}} \mid$ Goof $=\mathrm{S}=\left\{\Sigma\left[\mathrm{w}\left(\mathrm{F}_{\mathrm{o}}{ }^{2}-\mathrm{F}_{\mathrm{c}}{ }^{2}\right)^{2}\right] /(\mathrm{n}-\mathrm{p})\right\}^{1 / 2}$ where $\mathrm{n}$ is the number of reflections and $\mathrm{p}$ is the total number of parameters refined. 

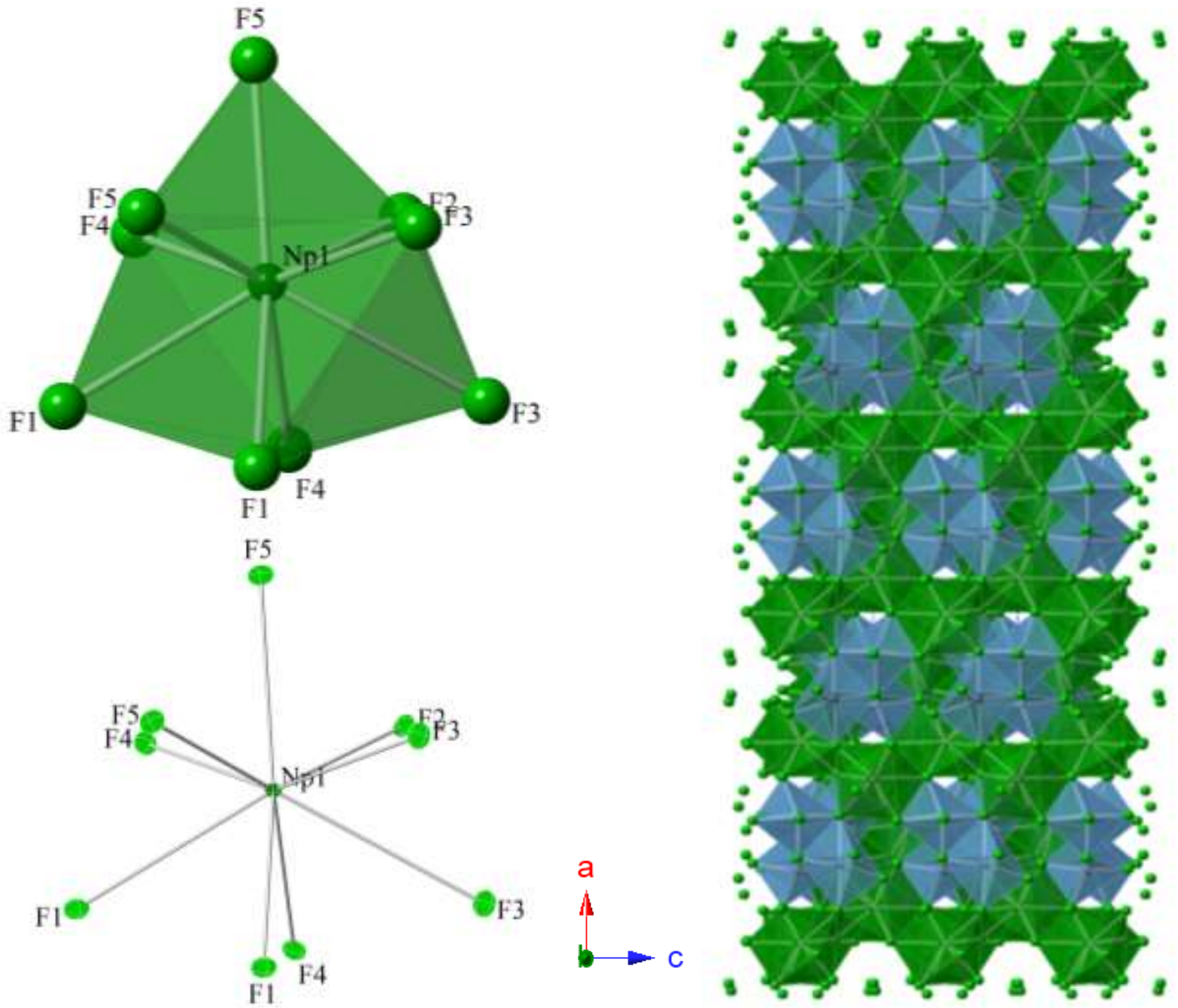

Figure S20. (Left) Local coordination environment of $\mathrm{Np}(1)$ in LiNpF5 with ball and stick polyhedron (top) and thermal ellipsoid plot plotted at the 50\% probability level (bottom), (Right) extended structure of $\mathbf{L i N p F}_{5}$ viewed down the $b$-axis with $\mathrm{Np}$ (dark green), $\mathrm{F}$ (green) and Li (blue).

Table S2. Bond lengths $[\AA]$ and angles $\left[{ }^{\circ}\right]$ for LiNpF 5 .

\begin{tabular}{llll}
\hline $\mathrm{Np}(1)-\mathrm{F}(3)$ & $2.227(2)$ & $\mathrm{Np}(1)-\mathrm{F}(1)$ & $2.297(2)$ \\
$\mathrm{Np}(1)-\mathrm{F}(2)$ & $2.251(2)$ & $\mathrm{Np}(1)-\mathrm{F}(4) \# 2$ & $2.306(2)$ \\
$\mathrm{Np}(1)-\mathrm{F}(5)$ & $2.251(2)$ & $\mathrm{Np}(1)-\mathrm{F}(1) \# 3$ & $2.434(2)$ \\
$\mathrm{Np}(1)-\mathrm{F}(5) \# 1$ & $2.284(2)$ & $\mathrm{Np}(1)-\mathrm{F}(3) \# 4$ & $2.536(2)$ \\
$\mathrm{Np}(1)-\mathrm{F}(4)$ & $2.290(2)$ & $\mathrm{Np}(1)-\mathrm{Li}(1) \# 5$ & $3.218(7)$
\end{tabular}




\begin{tabular}{|c|c|c|c|}
\hline $\mathrm{Np}(1)-\operatorname{Li}(1) \# 3$ & $3.480(7)$ & $\operatorname{Li}(1)-\mathrm{F}(3) \# 7$ & $2.106(8)$ \\
\hline Np(1)-Li(1)\#4 & $3.763(7)$ & $\mathrm{Li}(1)-\mathrm{F}(2) \# 8$ & $2.195(8)$ \\
\hline $\mathrm{Li}(1)-\mathrm{F}(1)$ & $1.863(7)$ & $\operatorname{Li}(1)-\operatorname{Li}(1) \# 9$ & $2.901(12)$ \\
\hline Li(1)-F(2)\#4 & $1.904(7)$ & $\operatorname{Li}(1)-\operatorname{Li}(1) \# 7$ & $2.901(12)$ \\
\hline Li(1)-F(2)\#6 & $1.965(7)$ & $\operatorname{Li}(1)-\operatorname{Li}(1) \# 10$ & $3.100(15)$ \\
\hline $\operatorname{Li}(1)-\mathrm{F}(4) \# 3$ & $2.096(7)$ & & \\
\hline $\mathrm{F}(3)-\mathrm{Np}(1)-\mathrm{F}(2)$ & $89.39(8)$ & $\mathrm{F}(2)-\mathrm{Np}(1)-\mathrm{F}(3) \# 4$ & $65.14(7)$ \\
\hline $\mathrm{F}(3)-\mathrm{Np}(1)-\mathrm{F}(5)$ & $78.47(8)$ & $\mathrm{F}(5)-\mathrm{Np}(1)-\mathrm{F}(3) \# 4$ & $132.66(7)$ \\
\hline $\mathrm{F}(2)-\mathrm{Np}(1)-\mathrm{F}(5)$ & $143.48(8)$ & $\mathrm{F}(5) \# 1-\mathrm{Np}(1)-\mathrm{F}(3) \# 4$ & $121.33(7)$ \\
\hline $\mathrm{F}(3)-\mathrm{Np}(1)-\mathrm{F}(5) \# 1$ & $80.57(8)$ & $\mathrm{F}(4)-\mathrm{Np}(1)-\mathrm{F}(3) \# 4$ & $126.76(7)$ \\
\hline $\mathrm{F}(2)-\mathrm{Np}(1)-\mathrm{F}(5) \# 1$ & $71.20(8)$ & $\mathrm{F}(1)-\mathrm{Np}(1)-\mathrm{F}(3) \# 4$ & 70.78(7) \\
\hline $\mathrm{F}(5)-\mathrm{Np}(1)-\mathrm{F}(5) \# 1$ & $72.87(9)$ & $\mathrm{F}(4) \# 2-\mathrm{Np}(1)-\mathrm{F}(3) \# 4$ & $63.01(7)$ \\
\hline $\mathrm{F}(3)-\mathrm{Np}(1)-\mathrm{F}(4)$ & $151.30(8)$ & $\mathrm{F}(1) \# 3-\mathrm{Np}(1)-\mathrm{F}(3) \# 4$ & $120.53(7)$ \\
\hline $\mathrm{F}(2)-\mathrm{Np}(1)-\mathrm{F}(4)$ & $74.43(8)$ & $\mathrm{F}(3)-\mathrm{Np}(1)-\mathrm{Li}(1) \# 5$ & $95.00(14)$ \\
\hline $\mathrm{F}(5)-\mathrm{Np}(1)-\mathrm{F}(4)$ & $100.42(7)$ & $\mathrm{F}(2)-\mathrm{Np}(1)-\mathrm{Li}(1) \# 5$ & $37.05(14)$ \\
\hline $\mathrm{F}(5) \# 1-\mathrm{Np}(1)-\mathrm{F}(4)$ & $71.90(8)$ & $\mathrm{F}(5)-\mathrm{Np}(1)-\mathrm{Li}(1) \# 5$ & $173.18(14)$ \\
\hline $\mathrm{F}(3)-\mathrm{Np}(1)-\mathrm{F}(1)$ & $81.54(8)$ & $\mathrm{F}(5) \# 1-\mathrm{Np}(1)-\mathrm{Li}(1) \# 5$ & $108.25(14)$ \\
\hline $\mathrm{F}(2)-\mathrm{Np}(1)-\mathrm{F}(1)$ & $133.94(7)$ & $\mathrm{F}(4)-\mathrm{Np}(1)-\mathrm{Li}(1) \# 5$ & $86.24(14)$ \\
\hline $\mathrm{F}(5)-\mathrm{Np}(1)-\mathrm{F}(1)$ & $78.59(7)$ & $\mathrm{F}(1)-\mathrm{Np}(1)-\mathrm{Li}(1) \# 5$ & $98.64(14)$ \\
\hline $\mathrm{F}(5) \# 1-\mathrm{Np}(1)-\mathrm{F}(1)$ & $148.76(7)$ & $\mathrm{F}(4) \# 2-\mathrm{Np}(1)-\mathrm{Li}(1) \# 5$ & $40.53(14)$ \\
\hline $\mathrm{F}(4)-\mathrm{Np}(1)-\mathrm{F}(1)$ & $126.70(8)$ & $\mathrm{F}(1) \# 3-\mathrm{Np}(1)-\mathrm{Li}(1) \# 5$ & $112.32(14)$ \\
\hline $\mathrm{F}(3)-\mathrm{Np}(1)-\mathrm{F}(4) \# 2$ & $125.19(8)$ & $\mathrm{F}(3) \# 4-\mathrm{Np}(1)-\mathrm{Li}(1) \# 5$ & $40.84(14)$ \\
\hline $\mathrm{F}(2)-\mathrm{Np}(1)-\mathrm{F}(4) \# 2$ & $70.95(8)$ & $F(3)-N p(1)-\operatorname{Li}(1) \# 3$ & $166.00(13)$ \\
\hline $\mathrm{F}(5)-\mathrm{Np}(1)-\mathrm{F}(4) \# 2$ & 143.07(8) & $\mathrm{F}(2)-\mathrm{Np}(1)-\mathrm{Li}(1) \# 3$ & $104.03(13)$ \\
\hline $\mathrm{F}(5) \# 1-\mathrm{Np}(1)-\mathrm{F}(4) \# 2$ & $133.17(7)$ & $\mathrm{F}(5)-\mathrm{Np}(1)-\mathrm{Li}(1) \# 3$ & $88.22(13)$ \\
\hline $\mathrm{F}(4)-\mathrm{Np}(1)-\mathrm{F}(4) \# 2$ & $72.20(10)$ & $\mathrm{F}(5) \# 1-\mathrm{Np}(1)-\mathrm{Li}(1) \# 3$ & $99.76(13)$ \\
\hline $\mathrm{F}(1)-\mathrm{Np}(1)-\mathrm{F}(4) \# 2$ & 77.95(7) & $\mathrm{F}(4)-\mathrm{Np}(1)-\mathrm{Li}(1) \# 3$ & $35.57(12)$ \\
\hline $\mathrm{F}(3)-\mathrm{Np}(1)-\mathrm{F}(1) \# 3$ & $137.57(7)$ & $\mathrm{F}(1)-\mathrm{Np}(1)-\mathrm{Li}(1) \# 3$ & $91.68(12)$ \\
\hline $\mathrm{F}(2)-\mathrm{Np}(1)-\mathrm{F}(1) \# 3$ & $131.90(7)$ & $\mathrm{F}(4) \# 2-\mathrm{Np}(1)-\mathrm{Li}(1) \# 3$ & $64.47(13)$ \\
\hline $\mathrm{F}(5)-\mathrm{Np}(1)-\mathrm{F}(1) \# 3$ & $72.16(8)$ & $\mathrm{F}(1) \# 3-\mathrm{Np}(1)-\mathrm{Li}(1) \# 3$ & $30.71(12)$ \\
\hline $\mathrm{F}(5) \# 1-\mathrm{Np}(1)-\mathrm{F}(1) \# 3$ & $117.58(7)$ & $\mathrm{F}(3) \# 4-\mathrm{Np}(1)-\mathrm{Li}(1) \# 3$ & $126.94(13)$ \\
\hline $\mathrm{F}(4)-\mathrm{Np}(1)-\mathrm{F}(1) \# 3$ & $65.84(7)$ & $\mathrm{Li}(1) \# 5-\mathrm{Np}(1)-\mathrm{Li}(1) \# 3$ & $98.1(2)$ \\
\hline $\mathrm{F}(1)-\mathrm{Np}(1)-\mathrm{F}(1) \# 3$ & $63.33(9)$ & $\mathrm{F}(3)-\mathrm{Np}(1)-\mathrm{Li}(1) \# 4$ & $66.72(12)$ \\
\hline $\mathrm{F}(4) \# 2-\mathrm{Np}(1)-\mathrm{F}(1) \# 3$ & 71.92(7) & $\mathrm{F}(2)-\mathrm{Np}(1)-\mathrm{Li}(1) \# 4$ & $22.92(13)$ \\
\hline $\mathrm{F}(3)-\mathrm{Np}(1)-\mathrm{F}(3) \# 4$ & $62.30(8)$ & $\mathrm{F}(5)-\mathrm{Np}(1)-\mathrm{Li}(1) \# 4$ & $128.94(12)$ \\
\hline
\end{tabular}




\begin{tabular}{|c|c|c|c|}
\hline $\mathrm{F}(5) \# 1-\mathrm{Np}(1)-\mathrm{Li}(1) \# 4$ & $65.73(12)$ & $\mathrm{F}(1)-\mathrm{Li}(1)-\mathrm{Li}(1) \# 10$ & $125.9(4)$ \\
\hline $\mathrm{F}(4)-\mathrm{Np}(1)-\mathrm{Li}(1) \# 4$ & $94.01(12)$ & $\mathrm{F}(2) \# 4-\mathrm{Li}(1)-\mathrm{Li}(1) \# 10$ & $85.0(2)$ \\
\hline $\mathrm{F}(1)-\mathrm{Np}(1)-\mathrm{Li}(1) \# 4$ & $128.25(12)$ & $\mathrm{F}(2) \# 6-\mathrm{Li}(1)-\mathrm{Li}(1) \# 10$ & $44.74(19)$ \\
\hline $\mathrm{F}(4) \# 2-\mathrm{Np}(1)-\mathrm{Li}(1) \# 4$ & $87.98(12)$ & $\mathrm{F}(4) \# 3-\mathrm{Li}(1)-\mathrm{Li}(1) \# 10$ & $95.3(2)$ \\
\hline $\mathrm{F}(1) \# 3-\mathrm{Np}(1)-\mathrm{Li}(1) \# 4$ & $154.78(12)$ & $\mathrm{F}(3) \# 7-\mathrm{Li}(1)-\mathrm{Li}(1) \# 10$ & $123.6(4)$ \\
\hline $\mathrm{F}(3) \# 4-\mathrm{Np}(1)-\mathrm{Li}(1) \# 4$ & $58.61(12)$ & $\mathrm{F}(2) \# 8-\mathrm{Li}(1)-\mathrm{Li}(1) \# 10$ & $39.06(18)$ \\
\hline $\mathrm{Li}(1) \# 5-\mathrm{Np}(1)-\mathrm{Li}(1) \# 4$ & $48.3(2)$ & Li(1)\#9-Li(1)-Li(1)\#10 & $57.71(14)$ \\
\hline $\mathrm{Li}(1) \# 3-\mathrm{Np}(1)-\mathrm{Li}(1) \# 4$ & $126.3(2)$ & $\mathrm{Li}(1) \# 7-\mathrm{Li}(1)-\mathrm{Li}(1) \# 10$ & $57.71(14)$ \\
\hline $\mathrm{F}(1)-\mathrm{Li}(1)-\mathrm{F}(2) \# 4$ & $110.3(4)$ & $\mathrm{F}(1)-\mathrm{Li}(1)-\mathrm{Np}(1) \# 6$ & $124.1(3)$ \\
\hline $\mathrm{F}(1)-\mathrm{Li}(1)-\mathrm{F}(2) \# 6$ & $158.5(4)$ & $\mathrm{F}(2) \# 4-\mathrm{Li}(1)-\mathrm{Np}(1) \# 6$ & $121.1(3)$ \\
\hline $\mathrm{F}(2) \# 4-\mathrm{Li}(1)-\mathrm{F}(2) \# 6$ & $89.4(3)$ & $\mathrm{F}(2) \# 6-\mathrm{Li}(1)-\mathrm{Np}(1) \# 6$ & $43.65(15)$ \\
\hline $\mathrm{F}(1)-\mathrm{Li}(1)-\mathrm{F}(4) \# 3$ & $80.7(3)$ & $\mathrm{F}(4) \# 3-\mathrm{Li}(1)-\mathrm{Np}(1) \# 6$ & $45.64(14)$ \\
\hline $\mathrm{F}(2) \# 4-\mathrm{Li}(1)-\mathrm{F}(4) \# 3$ & $166.2(4)$ & $\mathrm{F}(3) \# 7-\mathrm{Li}(1)-\mathrm{Np}(1) \# 6$ & $51.93(16)$ \\
\hline $\mathrm{F}(2) \# 6-\mathrm{Li}(1)-\mathrm{F}(4) \# 3$ & $81.2(3)$ & $\mathrm{F}(2) \# 8-\mathrm{Li}(1)-\mathrm{Np}(1) \# 6$ & $115.4(3)$ \\
\hline $\mathrm{F}(1)-\mathrm{Li}(1)-\mathrm{F}(3) \# 7$ & $107.3(4)$ & $\operatorname{Li}(1) \# 9-\operatorname{Li}(1)-\mathrm{Np}(1) \# 6$ & $133.2(2)$ \\
\hline $\mathrm{F}(2) \# 4-\mathrm{Li}(1)-\mathrm{F}(3) \# 7$ & $94.0(3)$ & $\mathrm{Li}(1) \# 7-\mathrm{Li}(1)-\mathrm{Np}(1) \# 6$ & $75.7(2)$ \\
\hline $\mathrm{F}(2) \# 6-\mathrm{Li}(1)-\mathrm{F}(3) \# 7$ & $78.9(3)$ & $\mathrm{Li}(1) \# 10-\mathrm{Li}(1)-\mathrm{Np}(1) \# 6$ & $80.9(2)$ \\
\hline $\mathrm{F}(4) \# 3-\mathrm{Li}(1)-\mathrm{F}(3) \# 7$ & $74.3(2)$ & $\mathrm{F}(1)-\mathrm{Li}(1)-\mathrm{Np}(1) \# 3$ & $41.84(17)$ \\
\hline $\mathrm{F}(1)-\mathrm{Li}(1)-\mathrm{F}(2) \# 8$ & $90.0(3)$ & $\mathrm{F}(2) \# 4-\mathrm{Li}(1)-\mathrm{Np}(1) \# 3$ & $152.2(3)$ \\
\hline $\mathrm{F}(2) \# 4-\mathrm{Li}(1)-\mathrm{F}(2) \# 8$ & $82.9(3)$ & $\mathrm{F}(2) \# 6-\mathrm{Li}(1)-\mathrm{Np}(1) \# 3$ & $118.1(3)$ \\
\hline $\mathrm{F}(2) \# 6-\mathrm{Li}(1)-\mathrm{F}(2) \# 8$ & $83.8(3)$ & $\mathrm{F}(4) \# 3-\mathrm{Li}(1)-\mathrm{Np}(1) \# 3$ & $39.46(14)$ \\
\hline $\mathrm{F}(4) \# 3-\mathrm{Li}(1)-\mathrm{F}(2) \# 8$ & $106.0(3)$ & $\mathrm{F}(3) \# 7-\mathrm{Li}(1)-\mathrm{Np}(1) \# 3$ & $95.2(2)$ \\
\hline $\mathrm{F}(3) \# 7-\mathrm{Li}(1)-\mathrm{F}(2) \# 8$ & $162.4(4)$ & $\mathrm{F}(2) \# 8-\mathrm{Li}(1)-\mathrm{Np}(1) \# 3$ & $95.5(2)$ \\
\hline $\mathrm{F}(1)-\operatorname{Li}(1)-\operatorname{Li}(1) \# 9$ & $99.2(4)$ & Li(1)\#9-Li(1)-Np(1)\#3 & $127.7(3)$ \\
\hline $\mathrm{F}(2) \# 4-\mathrm{Li}(1)-\mathrm{Li}(1) \# 9$ & $42.2(2)$ & Li(1)\#7-Li(1)-Np(1)\#3 & $158.7(3)$ \\
\hline $\mathrm{F}(2) \# 6-\mathrm{Li}(1)-\mathrm{Li}(1) \# 9$ & $89.6(2)$ & Li(1)\#10-Li(1)-Np(1)\#3 & $111.16(16)$ \\
\hline $\mathrm{F}(4) \# 3-\mathrm{Li}(1)-\mathrm{Li}(1) \# 9$ & $146.8(4)$ & $\mathrm{Np}(1) \# 6-\mathrm{Li}(1)-\mathrm{Np}(1) \# 3$ & $84.78(16)$ \\
\hline $\mathrm{F}(3) \# 7-\mathrm{Li}(1)-\mathrm{Li}(1) \# 9$ & $135.2(4)$ & $\mathrm{F}(1)-\mathrm{Li}(1)-\mathrm{Np}(1) \# 4$ & $85.7(2)$ \\
\hline $\mathrm{F}(2) \# 8-\mathrm{Li}(1)-\mathrm{Li}(1) \# 9$ & $41.02(13)$ & $\mathrm{F}(2) \# 4-\mathrm{Li}(1)-\mathrm{Np}(1) \# 4$ & $27.41(14)$ \\
\hline $\mathrm{F}(1)-\mathrm{Li}(1)-\mathrm{Li}(1) \# 7$ & $159.4(4)$ & $\mathrm{F}(2) \# 6-\mathrm{Li}(1)-\mathrm{Np}(1) \# 4$ & $115.3(3)$ \\
\hline $\mathrm{F}(2) \# 4-\mathrm{Li}(1)-\mathrm{Li}(1) \# 7$ & $49.2(3)$ & $\mathrm{F}(4) \# 3-\mathrm{Li}(1)-\mathrm{Np}(1) \# 4$ & $155.2(3)$ \\
\hline $\mathrm{F}(2) \# 6-\mathrm{Li}(1)-\mathrm{Li}(1) \# 7$ & $40.64(15)$ & $\mathrm{F}(3) \# 7-\mathrm{Li}(1)-\mathrm{Np}(1) \# 4$ & $90.2(2)$ \\
\hline $\mathrm{F}(4) \# 3-\mathrm{Li}(1)-\mathrm{Li}(1) \# 7$ & $119.9(3)$ & $\mathrm{F}(2) \# 8-\mathrm{Li}(1)-\mathrm{Np}(1) \# 4$ & $94.6(2)$ \\
\hline $\mathrm{F}(3) \# 7-\mathrm{Li}(1)-\mathrm{Li}(1) \# 7$ & $79.6(3)$ & $\mathrm{Li}(1) \# 9-\mathrm{Li}(1)-\mathrm{Np}(1) \# 4$ & $56.0(2)$ \\
\hline $\mathrm{F}(2) \# 8-\mathrm{Li}(1)-\mathrm{Li}(1) \# 7$ & $85.34(18)$ & Li(1)\#7-Li(1)-Np(1)\#4 & $74.7(3)$ \\
\hline Li(1)\#9-Li(1)-Li(1)\#7 & $64.6(3)$ & $\mathrm{Li}(1) \# 10-\mathrm{Li}(1)-\mathrm{Np}(1) \# 4$ & $109.50(14)$ \\
\hline
\end{tabular}




$\begin{array}{lllr}\mathrm{Np}(1) \# 6-\mathrm{Li}(1)-\mathrm{Np}(1) \# 4 & 135.4(2) & \mathrm{Li}(1) \# 5-\mathrm{F}(2)-\mathrm{Np}(1) & 99.3(2) \\ \mathrm{Np}(1) \# 3-\mathrm{Li}(1)-\mathrm{Np}(1) \# 4 & 126.3(2) & \mathrm{Li}(1) \# 1-\mathrm{F}(2)-\mathrm{Np}(1) & 134.6(2) \\ \mathrm{Li}(1)-\mathrm{F}(1)-\mathrm{Np}(1) & 129.9(2) & \mathrm{Li}(1) \# 9-\mathrm{F}(3)-\mathrm{Np}(1) & 133.9(2) \\ \mathrm{Li}(1)-\mathrm{F}(1)-\mathrm{Np}(1) \# 3 & 107.4(2) & \mathrm{Li}(1) \# 9-\mathrm{F}(3)-\mathrm{Np}(1) \# 4 & 87.2(2) \\ \mathrm{Np}(1)-\mathrm{F}(1)-\mathrm{Np}(1) \# 3 & 116.67(9) & \mathrm{Np}(1)-\mathrm{F}(3)-\mathrm{Np}(1) \# 4 & 117.71(8) \\ \mathrm{Li}(1) \# 4-\mathrm{F}(2)-\mathrm{Li}(1) \# 5 & 97.1(3) & \mathrm{Li}(1) \# 3-\mathrm{F}(4)-\mathrm{Np}(1) & 105.0(2) \\ \mathrm{Li}(1) \# 4-\mathrm{F}(2)-\mathrm{Li}(1) \# 1 & 89.8(3) & \mathrm{Li}(1) \# 3-\mathrm{F}(4)-\mathrm{Np}(1) \# 11 & 93.8(2) \\ \mathrm{Li}(1) \# 5-\mathrm{F}(2)-\mathrm{Li}(1) \# 1 & 96.2(3) & \mathrm{Np}(1)-\mathrm{F}(4)-\mathrm{Np}(1) \# 11 & 159.18(10) \\ \mathrm{Li}(1) \# 4-\mathrm{F}(2)-\mathrm{Np}(1) & 129.7(2) & \mathrm{Np}(1)-\mathrm{F}(5)-\mathrm{Np}(1) \# 8 & 152.88(1)\end{array}$

Symmetry transformations used to generate equivalent atoms:

$\# 1 \mathrm{y}+1 / 4,-\mathrm{x}+5 / 4, \mathrm{z}+1 / 4 \quad \# 2 \mathrm{y}-1 / 4,-\mathrm{x}+5 / 4,-\mathrm{z}+1 / 4 \quad \# 3-\mathrm{x}+1,-\mathrm{y}+1,-\mathrm{z} \quad \# 4-\mathrm{x}+1,-\mathrm{y}+1,-\mathrm{z}+1$

$\# 5-y+3 / 4, x+1 / 4, z+1 / 4 \quad \# 6 y-1 / 4,-x+3 / 4, z-1 / 4 \quad \# 7-y+3 / 4, x-1 / 4,-z+3 / 4 \quad \# 8-y+5 / 4, x-1 / 4, z-1 / 4$

$\# 9 \mathrm{y}+1 / 4,-\mathrm{x}+3 / 4,-\mathrm{z}+3 / 4 \quad \# 10-x+1,-y+1 / 2, \mathrm{z}+0 \quad \# 11-\mathrm{y}+5 / 4, x+1 / 4,-\mathrm{z}+1 / 4$
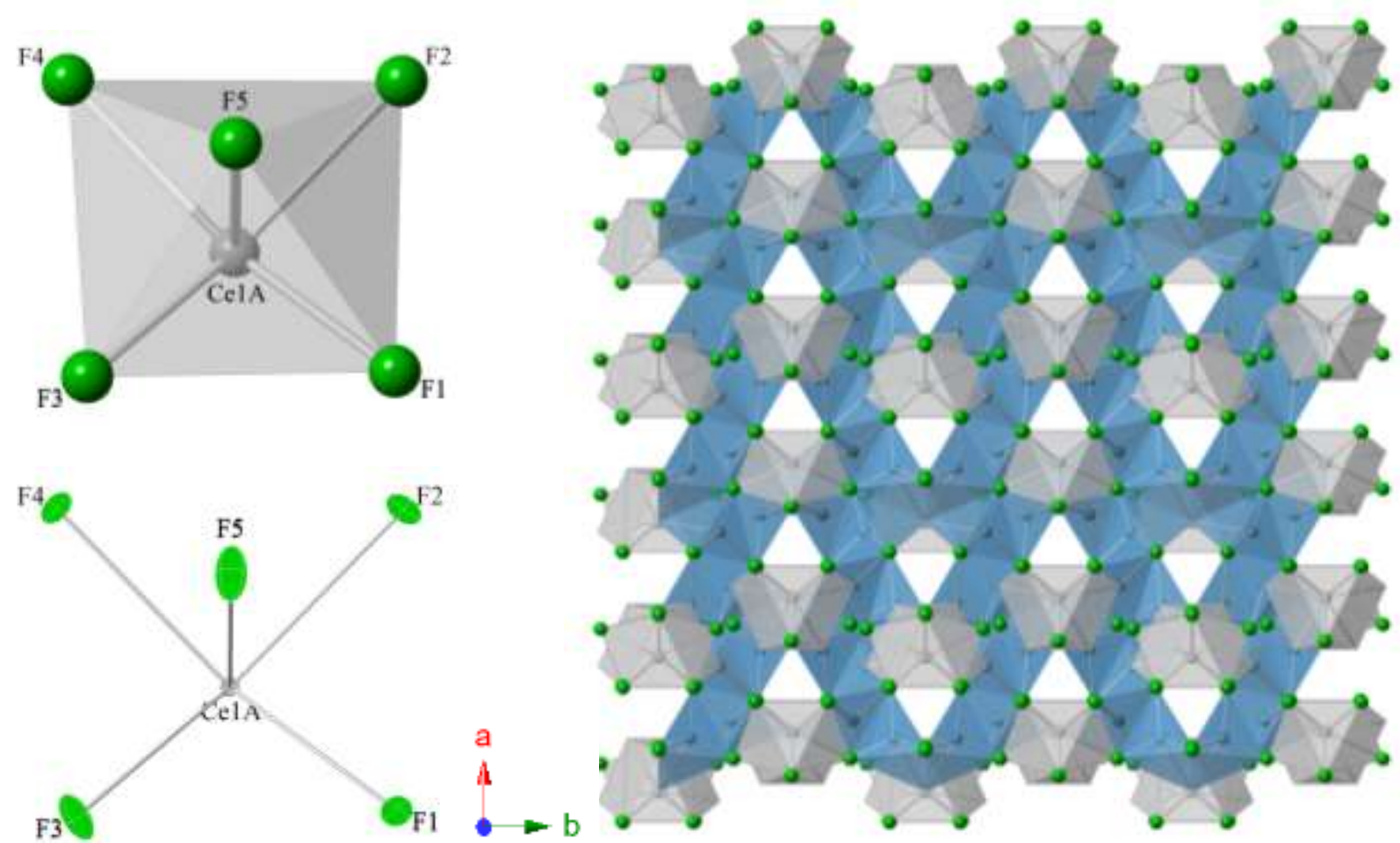

Figure S21. (Left) Local coordination environment of $\mathrm{Ce}(1 \mathrm{~A})$ in $\mathbf{L i} \mathbf{C i e F}_{8}$ with ball and stick polyhedron (top) and thermal ellipsoid plot plotted at the $50 \%$ probability level (bottom), (Right) extended structure of $\mathbf{L i}_{4} \mathbf{C e F} 8$ viewed down the $c$-axis with $\mathrm{Ce}$ (gray), F (green) and $\mathrm{Li}$ (blue). 
Table S3. Bond lengths $[\AA ̊]$ and angles $\left[{ }^{\circ}\right]$ for $\mathbf{L i}_{4} \mathrm{CeF}_{8}$.

\begin{tabular}{|c|c|c|c|}
\hline $\mathrm{Ce}(1 \mathrm{~A})-\mathrm{F}(2)$ & $2.200(5)$ & $\mathrm{Ce}(1 \mathrm{~B})-\mathrm{F}(3) \# 6$ & $2.283(9)$ \\
\hline $\mathrm{Ce}(1 \mathrm{~A})-\mathrm{F}(4)$ & $2.208(5)$ & $\mathrm{Ce}(1 \mathrm{~B})-\mathrm{F}(1)$ & $2.361(9)$ \\
\hline $\mathrm{Ce}(1 \mathrm{~A})-\mathrm{F}(3)$ & $2.232(4)$ & $\mathrm{Ce}(1 \mathrm{~B})-\mathrm{F}(1) \# 1$ & $2.361(9)$ \\
\hline $\mathrm{Ce}(1 \mathrm{~A})-\mathrm{F}(3) \# 1$ & $2.232(4)$ & $\mathrm{Ce}(1 \mathrm{~B})-\mathrm{Li}(1) \# 2$ & $3.56(3)$ \\
\hline $\mathrm{Ce}(1 \mathrm{~A})-\mathrm{F}(1)$ & $2.249(4)$ & $\mathrm{Ce}(1 \mathrm{~B})-\mathrm{Li}(1) \# 3$ & $3.56(3)$ \\
\hline $\mathrm{Ce}(1 \mathrm{~A})-\mathrm{F}(1) \# 1$ & $2.249(4)$ & $\operatorname{Li}(1)-\mathrm{F}(5)$ & $1.78(3)$ \\
\hline $\mathrm{Ce}(1 \mathrm{~A})-\mathrm{F}(5)$ & $2.372(4)$ & $\mathrm{Li}(1)-\mathrm{F}(2) \# 4$ & $1.86(3)$ \\
\hline $\mathrm{Ce}(1 \mathrm{~A})-\mathrm{F}(5) \# 1$ & $2.372(4)$ & $\operatorname{Li}(1)-\mathrm{F}(4) \# 7$ & $1.88(2)$ \\
\hline $\mathrm{Ce}(1 \mathrm{~A})-\mathrm{Li}(1) \# 2$ & $3.59(3)$ & $\operatorname{Li}(1)-\mathrm{F}(1) \# 10$ & $1.88(3)$ \\
\hline $\mathrm{Ce}(1 \mathrm{~A})-\mathrm{Li}(1) \# 3$ & $3.59(3)$ & $\operatorname{Li}(1)-\operatorname{Li}(1) \# 11$ & $2.30(5)$ \\
\hline $\mathrm{Ce}(1 \mathrm{~A})-\mathrm{Li}(1) \# 4$ & $3.70(2)$ & $\operatorname{Li}(1)-\operatorname{Li}(2)$ & $2.57(4)$ \\
\hline \multirow[t]{2}{*}{$\mathrm{Ce}(1 \mathrm{~A})-\mathrm{Li}(1) \# 5$} & $3.70(2)$ & $\operatorname{Li}(1)-\operatorname{Li}(2) \# 12$ & $3.17(4)$ \\
\hline & & Li(1)-Li(2)\#7 & $3.33(4)$ \\
\hline $\mathrm{Ce}(1 \mathrm{~B})-\mathrm{F}(2)$ & $2.099(11)$ & $\operatorname{Li}(1)-\operatorname{Li}(2) \# 4$ & $3.45(4)$ \\
\hline $\mathrm{Ce}(1 \mathrm{~B})-\mathrm{F}(4) \# 6$ & $2.163(11)$ & $\operatorname{Li}(2)-F(5)$ & $1.65(3)$ \\
\hline Ce(1B)-Li(1)\#7 & $2.26(3)$ & $\mathrm{Li}(2)-\mathrm{F}(3) \# 13$ & $1.85(3)$ \\
\hline Ce(1B)-Li(1)\#8 & $2.26(3)$ & $\mathrm{Li}(2)-\mathrm{F}(1) \# 14$ & $1.92(3)$ \\
\hline $\mathrm{Ce}(1 \mathrm{~B})-\mathrm{Li}(2) \# 8$ & $2.27(3)$ & $\mathrm{Li}(2)-\mathrm{F}(3) \# 7$ & $1.97(3)$ \\
\hline Ce(1B)-Li(2)\#7 & $2.27(3)$ & $\operatorname{Li}(2)-\operatorname{Li}(2) \# 15$ & $2.64(5)$ \\
\hline $\mathrm{Ce}(1 \mathrm{~B})-\mathrm{F}(3) \# 9$ & 2.283(9) & & \\
\hline $\mathrm{F}(2)-\mathrm{Ce}(1 \mathrm{~A})-\mathrm{F}(4)$ & $88.5(2)$ & $\mathrm{F}(3) \# 1-\mathrm{Ce}(1 \mathrm{~A})-\mathrm{F}(1) \# 1$ & $119.79(15)$ \\
\hline $\mathrm{F}(2)-\mathrm{Ce}(1 \mathrm{~A})-\mathrm{F}(3)$ & $142.48(10)$ & $\mathrm{F}(1)-\mathrm{Ce}(1 \mathrm{~A})-\mathrm{F}(1) \# 1$ & $70.44(19)$ \\
\hline $\mathrm{F}(4)-\mathrm{Ce}(1 \mathrm{~A})-\mathrm{F}(3)$ & $86.88(16)$ & $\mathrm{F}(2)-\mathrm{Ce}(1 \mathrm{~A})-\mathrm{F}(5)$ & $72.18(11)$ \\
\hline $\mathrm{F}(2)-\mathrm{Ce}(1 \mathrm{~A})-\mathrm{F}(3) \# 1$ & $142.48(10)$ & $\mathrm{F}(4)-\mathrm{Ce}(1 \mathrm{~A})-\mathrm{F}(5)$ & $72.43(11)$ \\
\hline $\mathrm{F}(4)-\mathrm{Ce}(1 \mathrm{~A})-\mathrm{F}(3) \# 1$ & $86.88(16)$ & $\mathrm{F}(3)-\mathrm{Ce}(1 \mathrm{~A})-\mathrm{F}(5)$ & $70.92(15)$ \\
\hline $\mathrm{F}(3)-\mathrm{Ce}(1 \mathrm{~A})-\mathrm{F}(3) \# 1$ & $74.4(2)$ & $\mathrm{F}(3) \# 1-\mathrm{Ce}(1 \mathrm{~A})-\mathrm{F}(5)$ & $140.16(14)$ \\
\hline $\mathrm{F}(2)-\mathrm{Ce}(1 \mathrm{~A})-\mathrm{F}(1)$ & $83.70(15)$ & $\mathrm{F}(1)-\mathrm{Ce}(1 \mathrm{~A})-\mathrm{F}(5)$ & $136.59(13)$ \\
\hline $\mathrm{F}(4)-\mathrm{Ce}(1 \mathrm{~A})-\mathrm{F}(1)$ & $143.74(10)$ & $\mathrm{F}(1) \# 1-\mathrm{Ce}(1 \mathrm{~A})-\mathrm{F}(5)$ & $71.46(14)$ \\
\hline $\mathrm{F}(3)-\mathrm{Ce}(1 \mathrm{~A})-\mathrm{F}(1)$ & $119.79(15)$ & $\mathrm{F}(2)-\mathrm{Ce}(1 \mathrm{~A})-\mathrm{F}(5) \# 1$ & $72.18(11)$ \\
\hline $\mathrm{F}(3) \# 1-\mathrm{Ce}(1 \mathrm{~A})-\mathrm{F}(1)$ & $78.45(15)$ & $\mathrm{F}(4)-\mathrm{Ce}(1 \mathrm{~A})-\mathrm{F}(5) \# 1$ & $72.43(11)$ \\
\hline $\mathrm{F}(2)-\mathrm{Ce}(1 \mathrm{~A})-\mathrm{F}(1) \# 1$ & $83.71(15)$ & $\mathrm{F}(3)-\mathrm{Ce}(1 \mathrm{~A})-\mathrm{F}(5) \# 1$ & $140.16(14)$ \\
\hline $\mathrm{F}(4)-\mathrm{Ce}(1 \mathrm{~A})-\mathrm{F}(1) \# 1$ & $143.74(10)$ & $\mathrm{F}(3) \# 1-\mathrm{Ce}(1 \mathrm{~A})-\mathrm{F}(5) \# 1$ & $70.92(15)$ \\
\hline $\mathrm{F}(3)-\mathrm{Ce}(1 \mathrm{~A})-\mathrm{F}(1) \# 1$ & $78.44(15)$ & $\mathrm{F}(1)-\mathrm{Ce}(1 \mathrm{~A})-\mathrm{F}(5) \# 1$ & $71.46(14)$ \\
\hline
\end{tabular}




\begin{tabular}{|c|c|c|c|}
\hline $\mathrm{F}(1) \# 1-\mathrm{Ce}(1 \mathrm{~A})-\mathrm{F}(5) \# 1$ & $136.59(13)$ & $\mathrm{F}(5) \# 1-\mathrm{Ce}(1 \mathrm{~A})-\mathrm{Li}(1) \# 5$ & $60.7(4)$ \\
\hline $\mathrm{F}(5)-\mathrm{Ce}(1 \mathrm{~A})-\mathrm{F}(5) \# 1$ & $129.8(2)$ & $\mathrm{Li}(1) \# 2-\mathrm{Ce}(1 \mathrm{~A})-\mathrm{Li}(1) \# 5$ & $133.2(7)$ \\
\hline $\mathrm{F}(2)-\mathrm{Ce}(1 \mathrm{~A})-\mathrm{Li}(1) \# 2$ & $105.5(4)$ & Li(1)\#3-Ce(1A)-Li(1)\#5 & $152.2(6)$ \\
\hline $\mathrm{F}(4)-\mathrm{Ce}(1 \mathrm{~A})-\mathrm{Li}(1) \# 2$ & $156.3(4)$ & Li(1)\#4-Ce(1A)-Li(1)\#5 & $36.3(8)$ \\
\hline $\mathrm{F}(3)-\mathrm{Ce}(1 \mathrm{~A})-\mathrm{Li}(1) \# 2$ & $92.9(4)$ & & \\
\hline $\mathrm{F}(3) \# 1-\mathrm{Ce}(1 \mathrm{~A})-\mathrm{Li}(1) \# 2$ & $70.3(4)$ & $\mathrm{F}(2)-\mathrm{Ce}(1 \mathrm{~B})-\mathrm{F}(4) \# 6$ & $84.0(4)$ \\
\hline $\mathrm{F}(1)-\mathrm{Ce}(1 \mathrm{~A})-\mathrm{Li}(1) \# 2$ & $27.0(4)$ & $\mathrm{F}(2)-\mathrm{Ce}(1 \mathrm{~B})-\mathrm{Li}(1) \# 7$ & $50.3(6)$ \\
\hline $\mathrm{F}(1) \# 1-\mathrm{Ce}(1 \mathrm{~A})-\mathrm{Li}(1) \# 2$ & $58.6(4)$ & $\mathrm{F}(4) \# 6-\mathrm{Ce}(1 \mathrm{~B})-\mathrm{Li}(1) \# 7$ & $50.2(7)$ \\
\hline $\mathrm{F}(5)-\mathrm{Ce}(1 \mathrm{~A})-\mathrm{Li}(1) \# 2$ & $129.7(4)$ & $\mathrm{F}(2)-\mathrm{Ce}(1 \mathrm{~B})-\mathrm{Li}(1) \# 8$ & $50.3(6)$ \\
\hline $\mathrm{F}(5) \# 1-\mathrm{Ce}(1 \mathrm{~A})-\mathrm{Li}(1) \# 2$ & $93.3(4)$ & $\mathrm{F}(4) \# 6-\mathrm{Ce}(1 \mathrm{~B})-\mathrm{Li}(1) \# 8$ & $50.2(7)$ \\
\hline $\mathrm{F}(2)-\mathrm{Ce}(1 \mathrm{~A})-\mathrm{Li}(1) \# 3$ & $105.5(4)$ & Li(1)\#7-Ce(1B)-Li(1)\#8 & $61.2(14)$ \\
\hline $\mathrm{F}(4)-\mathrm{Ce}(1 \mathrm{~A})-\mathrm{Li}(1) \# 3$ & $156.3(4)$ & $\mathrm{F}(2)-\mathrm{Ce}(1 \mathrm{~B})-\mathrm{Li}(2) \# 8$ & $94.8(6)$ \\
\hline $\mathrm{F}(3)-\mathrm{Ce}(1 \mathrm{~A})-\mathrm{Li}(1) \# 3$ & $70.3(4)$ & $\mathrm{F}(4) \# 6-\mathrm{Ce}(1 \mathrm{~B})-\mathrm{Li}(2) \# 8$ & 99.2(9) \\
\hline F(3)\#1-Ce(1A)-Li(1)\#3 & $92.9(4)$ & Li(1)\#7-Ce(1B)-Li(2)\#8 & $130.0(10)$ \\
\hline $\mathrm{F}(1)-\mathrm{Ce}(1 \mathrm{~A})-\mathrm{Li}(1) \# 3$ & $58.6(4)$ & Li(1)\#8-Ce(1B)-Li(2)\#8 & $68.9(10)$ \\
\hline $\mathrm{F}(1) \# 1-\mathrm{Ce}(1 \mathrm{~A})-\mathrm{Li}(1) \# 3$ & $27.0(4)$ & $\mathrm{F}(2)-\mathrm{Ce}(1 \mathrm{~B})-\mathrm{Li}(2) \# 7$ & $94.8(6)$ \\
\hline $\mathrm{F}(5)-\mathrm{Ce}(1 \mathrm{~A})-\mathrm{Li}(1) \# 3$ & $93.3(4)$ & $\mathrm{F}(4) \# 6-\mathrm{Ce}(1 \mathrm{~B})-\mathrm{Li}(2) \# 7$ & 99.2(9) \\
\hline $\mathrm{F}(5) \# 1-\mathrm{Ce}(1 \mathrm{~A})-\mathrm{Li}(1) \# 3$ & $129.7(4)$ & Li(1)\#7-Ce(1B)-Li(2)\#7 & $68.9(10)$ \\
\hline $\operatorname{Li}(1) \# 2-\operatorname{Ce}(1 \mathrm{~A})-\mathrm{Li}(1) \# 3$ & $37.4(8)$ & $\mathrm{Li}(1) \# 8-\mathrm{Ce}(1 \mathrm{~B})-\mathrm{Li}(2) \# 7$ & $130.0(10)$ \\
\hline $\mathrm{F}(2)-\mathrm{Ce}(1 \mathrm{~A})-\mathrm{Li}(1) \# 4$ & $102.3(5)$ & $\mathrm{Li}(2) \# 8-\mathrm{Ce}(1 \mathrm{~B})-\mathrm{Li}(2) \# 7$ & $160.0(16)$ \\
\hline $\mathrm{F}(4)-\mathrm{Ce}(1 \mathrm{~A})-\mathrm{Li}(1) \# 4$ & $23.0(5)$ & $\mathrm{F}(2)-\mathrm{Ce}(1 \mathrm{~B})-\mathrm{F}(3) \# 9$ & $142.6(2)$ \\
\hline $\mathrm{F}(3)-\mathrm{Ce}(1 \mathrm{~A})-\mathrm{Li}(1) \# 4$ & $64.7(5)$ & $\mathrm{F}(4) \# 6-\mathrm{Ce}(1 \mathrm{~B})-\mathrm{F}(3) \# 9$ & $86.7(4)$ \\
\hline F(3)\#1-Ce(1A)-Li(1)\#4 & 87.1(4) & $\mathrm{Li}(1) \# 7-\mathrm{Ce}(1 \mathrm{~B})-\mathrm{F}(3) \# 9$ & $136.6(8)$ \\
\hline $\mathrm{F}(1)-\mathrm{Ce}(1 \mathrm{~A})-\mathrm{Li}(1) \# 4$ & $162.3(4)$ & $\mathrm{Li}(1) \# 8-\mathrm{Ce}(1 \mathrm{~B})-\mathrm{F}(3) \# 9$ & $97.2(7)$ \\
\hline F(1)\#1-Ce(1A)-Li(1)\#4 & $126.4(4)$ & $\mathrm{Li}(2) \# 8-\mathrm{Ce}(1 \mathrm{~B})-\mathrm{F}(3) \# 9$ & $51.2(6)$ \\
\hline $\mathrm{F}(5)-\mathrm{Ce}(1 \mathrm{~A})-\mathrm{Li}(1) \# 4$ & $60.7(4)$ & $\mathrm{Li}(2) \# 7-\mathrm{Ce}(1 \mathrm{~B})-\mathrm{F}(3) \# 9$ & $122.5(7)$ \\
\hline F(5)\#1-Ce(1A)-Li(1)\#4 & $94.3(4)$ & $\mathrm{F}(2)-\mathrm{Ce}(1 \mathrm{~B})-\mathrm{F}(3) \# 6$ & $142.6(2)$ \\
\hline Li(1)\#2-Ce(1A)-Li(1)\#4 & $152.2(6)$ & $\mathrm{F}(4) \# 6-\mathrm{Ce}(1 \mathrm{~B})-\mathrm{F}(3) \# 6$ & $86.7(4)$ \\
\hline Li(1)\#3-Ce(1A)-Li(1)\#4 & $133.2(7)$ & Li(1)\#7-Ce(1B)-F(3)\#6 & $97.2(7)$ \\
\hline $\mathrm{F}(2)-\mathrm{Ce}(1 \mathrm{~A})-\mathrm{Li}(1) \# 5$ & $102.3(5)$ & Li(1)\#8-Ce(1B)-F(3)\#6 & $136.6(8)$ \\
\hline $\mathrm{F}(4)-\mathrm{Ce}(1 \mathrm{~A})-\mathrm{Li}(1) \# 5$ & $23.0(5)$ & Li(2)\#8-Ce(1B)-F(3)\#6 & $122.5(7)$ \\
\hline $\mathrm{F}(3)-\mathrm{Ce}(1 \mathrm{~A})-\mathrm{Li}(1) \# 5$ & 87.1(4) & $\mathrm{Li}(2) \# 7-\mathrm{Ce}(1 \mathrm{~B})-\mathrm{F}(3) \# 6$ & $51.2(6)$ \\
\hline F(3)\#1-Ce(1A)-Li(1)\#5 & $64.7(5)$ & $\mathrm{F}(3) \# 9-\mathrm{Ce}(1 \mathrm{~B})-\mathrm{F}(3) \# 6$ & $72.5(4)$ \\
\hline $\mathrm{F}(1)-\mathrm{Ce}(1 \mathrm{~A})-\mathrm{Li}(1) \# 5$ & $126.4(4)$ & $\mathrm{F}(2)-\mathrm{Ce}(1 \mathrm{~B})-\mathrm{F}(1)$ & $83.2(3)$ \\
\hline $\mathrm{F}(1) \# 1-\mathrm{Ce}(1 \mathrm{~A})-\mathrm{Li}(1) \# 5$ & $162.3(4)$ & $\mathrm{F}(4) \# 6-\mathrm{Ce}(1 \mathrm{~B})-\mathrm{F}(1)$ & 144.1(2) \\
\hline $\mathrm{F}(5)-\mathrm{Ce}(1 \mathrm{~A})-\mathrm{Li}(1) \# 5$ & $94.3(4)$ & $\mathrm{Li}(1) \# 7-\mathrm{Ce}(1 \mathrm{~B})-\mathrm{F}(1)$ & $133.0(7)$ \\
\hline
\end{tabular}




\begin{tabular}{|c|c|c|c|}
\hline $\mathrm{Li}(1) \# 8-\mathrm{Ce}(1 \mathrm{~B})-\mathrm{F}(1)$ & $97.0(7)$ & $\mathrm{F}(5)-\mathrm{Li}(1)-\mathrm{F}(4) \# 7$ & $118.7(13)$ \\
\hline $\mathrm{Li}(2) \# 8-\mathrm{Ce}(1 \mathrm{~B})-\mathrm{F}(1)$ & $48.9(9)$ & $\mathrm{F}(2) \# 4-\mathrm{Li}(1)-\mathrm{F}(4) \# 7$ & $99.5(12)$ \\
\hline $\mathrm{Li}(2) \# 7-\mathrm{Ce}(1 \mathrm{~B})-\mathrm{F}(1)$ & 115.2(9) & $\mathrm{F}(5)-\mathrm{Li}(1)-\mathrm{F}(1) \# 10$ & $110.7(14)$ \\
\hline $\mathrm{F}(3) \# 9-\mathrm{Ce}(1 \mathrm{~B})-\mathrm{F}(1)$ & $83.4(3)$ & $\mathrm{F}(2) \# 4-\mathrm{Li}(1)-\mathrm{F}(1) \# 10$ & $108.8(13)$ \\
\hline $\mathrm{F}(3) \# 6-\mathrm{Ce}(1 \mathrm{~B})-\mathrm{F}(1)$ & $122.3(5)$ & $\mathrm{F}(4) \# 7-\mathrm{Li}(1)-\mathrm{F}(1) \# 10$ & $99.1(14)$ \\
\hline $\mathrm{F}(2)-\mathrm{Ce}(1 \mathrm{~B})-\mathrm{F}(1) \# 1$ & $83.2(3)$ & $\mathrm{F}(5)-\mathrm{Li}(1)-\mathrm{Ce}(1 \mathrm{~B}) \# 4$ & $95.4(14)$ \\
\hline $\mathrm{F}(4) \# 6-\mathrm{Ce}(1 \mathrm{~B})-\mathrm{F}(1) \# 1$ & $144.1(2)$ & $\mathrm{F}(2) \# 4-\mathrm{Li}(1)-\mathrm{Ce}(1 \mathrm{~B}) \# 4$ & $60.3(9)$ \\
\hline $\mathrm{Li}(1) \# 7-\mathrm{Ce}(1 \mathrm{~B})-\mathrm{F}(1) \# 1$ & $97.0(7)$ & F(4)\#7-Li(1)-Ce(1B)\#4 & $62.1(8)$ \\
\hline $\mathrm{Li}(1) \# 8-\mathrm{Ce}(1 \mathrm{~B})-\mathrm{F}(1) \# 1$ & $133.0(7)$ & $\mathrm{F}(1) \# 10-\mathrm{Li}(1)-\mathrm{Ce}(1 \mathrm{~B}) \# 4$ & $153.4(15)$ \\
\hline $\mathrm{Li}(2) \# 8-\mathrm{Ce}(1 \mathrm{~B})-\mathrm{F}(1) \# 1$ & 115.2(9) & $\mathrm{F}(5)-\mathrm{Li}(1)-\mathrm{Li}(1) \# 11$ & $154.8(10)$ \\
\hline $\mathrm{Li}(2) \# 7-\mathrm{Ce}(1 \mathrm{~B})-\mathrm{F}(1) \# 1$ & $48.9(9)$ & $\mathrm{F}(2) \# 4-\mathrm{Li}(1)-\mathrm{Li}(1) \# 11$ & $51.7(8)$ \\
\hline $\mathrm{F}(3) \# 9-\mathrm{Ce}(1 \mathrm{~B})-\mathrm{F}(1) \# 1$ & $122.3(5)$ & $\mathrm{F}(4) \# 7-\mathrm{Li}(1)-\mathrm{Li}(1) \# 11$ & $52.2(7)$ \\
\hline $\mathrm{F}(3) \# 6-\mathrm{Ce}(1 \mathrm{~B})-\mathrm{F}(1) \# 1$ & $83.4(3)$ & $\mathrm{F}(1) \# 10-\mathrm{Li}(1)-\mathrm{Li}(1) \# 11$ & $94.4(8)$ \\
\hline $\mathrm{F}(1)-\mathrm{Ce}(1 \mathrm{~B})-\mathrm{F}(1) \# 1$ & $66.7(3)$ & $\mathrm{F}(5)-\mathrm{Li}(1)-\mathrm{Li}(2)$ & $39.8(9)$ \\
\hline $\mathrm{F}(2)-\mathrm{Ce}(1 \mathrm{~B})-\mathrm{Li}(1) \# 2$ & 109.1(6) & $\mathrm{F}(2) \# 4-\mathrm{Li}(1)-\mathrm{Li}(2)$ & $92.0(14)$ \\
\hline $\mathrm{F}(4) \# 6-\mathrm{Ce}(1 \mathrm{~B})-\mathrm{Li}(1) \# 2$ & $156.5(5)$ & $\mathrm{F}(4) \# 7-\mathrm{Li}(1)-\mathrm{Li}(2)$ & $97.8(13)$ \\
\hline $\mathrm{Li}(1) \# 7-\mathrm{Ce}(1 \mathrm{~B})-\mathrm{Li}(1) \# 2$ & $152.4(4)$ & $\mathrm{F}(1) \# 10-\mathrm{Li}(1)-\mathrm{Li}(2)$ & $150.5(15)$ \\
\hline $\mathrm{Li}(1) \# 8-\mathrm{Ce}(1 \mathrm{~B})-\mathrm{Li}(1) \# 2$ & $123.8(9)$ & $\mathrm{Ce}(1 \mathrm{~B}) \# 4-\mathrm{Li}(1)-\mathrm{Li}(2)$ & $55.8(10)$ \\
\hline Li(2)\#8-Ce(1B)-Li(1)\#2 & $61.2(9)$ & Li(1)\#11-Li(1)-Li(2) & $115.1(9)$ \\
\hline Li(2)\#7-Ce(1B)-Li(1)\#2 & $99.0(10)$ & $\mathrm{F}(5)-\mathrm{Li}(1)-\mathrm{Li}(2) \# 12$ & $92.7(11)$ \\
\hline $\mathrm{F}(3) \# 9-\mathrm{Ce}(1 \mathrm{~B})-\mathrm{Li}(1) \# 2$ & $71.0(5)$ & $\mathrm{F}(2) \# 4-\mathrm{Li}(1)-\mathrm{Li}(2) \# 12$ & $94.5(10)$ \\
\hline $\mathrm{F}(3) \# 6-\mathrm{Ce}(1 \mathrm{~B})-\mathrm{Li}(1) \# 2$ & $93.3(5)$ & $\mathrm{F}(4) \# 7-\mathrm{Li}(1)-\mathrm{Li}(2) \# 12$ & $132.7(15)$ \\
\hline $\mathrm{F}(1)-\mathrm{Ce}(1 \mathrm{~B})-\mathrm{Li}(1) \# 2$ & $29.1(5)$ & $\mathrm{F}(1) \# 10-\mathrm{Li}(1)-\mathrm{Li}(2) \# 12$ & $34.0(7)$ \\
\hline $\mathrm{F}(1) \# 1-\mathrm{Ce}(1 \mathrm{~B})-\mathrm{Li}(1) \# 2$ & $58.8(5)$ & $\mathrm{Li}(1) \# 11-\mathrm{Li}(1)-\mathrm{Li}(2) \# 12$ & 110.1(7) \\
\hline $\mathrm{F}(2)-\mathrm{Ce}(1 \mathrm{~B})-\mathrm{Li}(1) \# 3$ & 109.1(6) & $\mathrm{Li}(2)-\mathrm{Li}(1)-\mathrm{Li}(2) \# 12$ & $126.7(14)$ \\
\hline $\mathrm{F}(4) \# 6-\mathrm{Ce}(1 \mathrm{~B})-\mathrm{Li}(1) \# 3$ & $156.5(5)$ & $\mathrm{F}(5)-\operatorname{Li}(1)-\operatorname{Li}(2) \# 7$ & $70.1(9)$ \\
\hline Li(1)\#7-Ce(1B)-Li(1)\#3 & $123.8(9)$ & $\mathrm{F}(2) \# 4-\mathrm{Li}(1)-\mathrm{Li}(2) \# 7$ & $168.2(13)$ \\
\hline Li(1)\#8-Ce(1B)-Li(1)\#3 & $152.4(4)$ & $\mathrm{F}(4) \# 7-\mathrm{Li}(1)-\mathrm{Li}(2) \# 7$ & $68.8(9)$ \\
\hline $\mathrm{Li}(2) \# 8-\mathrm{Ce}(1 \mathrm{~B})-\mathrm{Li}(1) \# 3$ & $99.0(10)$ & $\mathrm{F}(1) \# 10-\mathrm{Li}(1)-\mathrm{Li}(2) \# 7$ & $73.8(10)$ \\
\hline Li(2)\#7-Ce(1B)-Li(1)\#3 & $61.2(9)$ & Li(1)\#11-Li(1)-Li(2)\#7 & $117.1(7)$ \\
\hline $\mathrm{F}(3) \# 9-\mathrm{Ce}(1 \mathrm{~B})-\mathrm{Li}(1) \# 3$ & $93.3(6)$ & $\mathrm{Li}(2)-\mathrm{Li}(1)-\mathrm{Li}(2) \# 7$ & $90.1(12)$ \\
\hline $\mathrm{F}(3) \# 6-\mathrm{Ce}(1 \mathrm{~B})-\mathrm{Li}(1) \# 3$ & $71.0(5)$ & $\mathrm{Li}(2) \# 12-\mathrm{Li}(1)-\mathrm{Li}(2) \# 7$ & $93.5(11)$ \\
\hline $\mathrm{F}(1)-\mathrm{Ce}(1 \mathrm{~B})-\mathrm{Li}(1) \# 3$ & $58.8(5)$ & $\mathrm{F}(5)-\mathrm{Li}(1)-\mathrm{Li}(2) \# 4$ & $71.8(9)$ \\
\hline $\mathrm{F}(1) \# 1-\mathrm{Ce}(1 \mathrm{~B})-\mathrm{Li}(1) \# 3$ & $29.1(5)$ & $\mathrm{F}(2) \# 4-\mathrm{Li}(1)-\mathrm{Li}(2) \# 4$ & $69.8(9)$ \\
\hline $\mathrm{Li}(1) \# 2-\mathrm{Ce}(1 \mathrm{~B})-\mathrm{Li}(1) \# 3$ & $37.8(8)$ & $\mathrm{F}(4) \# 7-\operatorname{Li}(1)-\mathrm{Li}(2) \# 4$ & $168.3(13)$ \\
\hline $\mathrm{F}(5)-\mathrm{Li}(1)-\mathrm{F}(2) \# 4$ & $118.0(16)$ & $\mathrm{F}(1) \# 10-\mathrm{Li}(1)-\mathrm{Li}(2) \# 4$ & $80.8(9)$ \\
\hline
\end{tabular}




\begin{tabular}{|c|c|c|c|}
\hline $\operatorname{Li}(1) \# 11-\operatorname{Li}(1)-\operatorname{Li}(2) \# 4$ & $116.1(7)$ & $\mathrm{Ce}(1 \mathrm{~B}) \# 4-\mathrm{Li}(2)-\mathrm{Li}(1)$ & $55.3(9)$ \\
\hline $\mathrm{Li}(2)-\mathrm{Li}(1)-\mathrm{Li}(2) \# 4$ & $87.4(10)$ & $\mathrm{F}(5)-\mathrm{Li}(2)-\mathrm{Li}(2) \# 15$ & $130(2)$ \\
\hline $\operatorname{Li}(2) \# 12-\operatorname{Li}(1)-\operatorname{Li}(2) \# 4$ & $46.9(8)$ & $\mathrm{F}(3) \# 13-\mathrm{Li}(2)-\operatorname{Li}(2) \# 15$ & $48.0(9)$ \\
\hline Li(2)\#7-Li(1)-Li(2)\#4 & $121.9(12)$ & $\mathrm{F}(1) \# 14-\mathrm{Li}(2)-\operatorname{Li}(2) \# 15$ & $105.2(18)$ \\
\hline $\mathrm{F}(5)-\mathrm{Li}(1)-\mathrm{Ce}(1 \mathrm{~B}) \# 3$ & 131.1(12) & $\mathrm{F}(3) \# 7-\operatorname{Li}(2)-\operatorname{Li}(2) \# 15$ & $44.4(8)$ \\
\hline $\mathrm{F}(2) \# 4-\mathrm{Li}(1)-\mathrm{Ce}(1 \mathrm{~B}) \# 3$ & $71.3(8)$ & $\operatorname{Li}(1)-\operatorname{Li}(2)-\operatorname{Li}(2) \# 15$ & $138(2)$ \\
\hline $\mathrm{F}(4) \# 7-\mathrm{Li}(1)-\mathrm{Ce}(1 \mathrm{~B}) \# 3$ & $105.1(11)$ & $\mathrm{F}(5)-\mathrm{Li}(2)-\operatorname{Li}(1) \# 13$ & $157.1(15)$ \\
\hline $\mathrm{F}(1) \# 10-\mathrm{Li}(1)-\mathrm{Ce}(1 \mathrm{~B}) \# 3$ & $37.5(5)$ & $\mathrm{F}(3) \# 13-\operatorname{Li}(2)-\operatorname{Li}(1) \# 13$ & $71.0(11)$ \\
\hline Li(1)\#11-Li(1)-Ce(1B)\#3 & $71.1(4)$ & $\mathrm{F}(1) \# 14-\operatorname{Li}(2)-\operatorname{Li}(1) \# 13$ & $33.3(7)$ \\
\hline $\mathrm{Li}(2)-\mathrm{Li}(1)-\mathrm{Ce}(1 \mathrm{~B}) \# 3$ & $153.4(12)$ & $\mathrm{F}(3) \# 7-\operatorname{Li}(2)-\operatorname{Li}(1) \# 13$ & $83.9(10)$ \\
\hline $\mathrm{Li}(2) \# 12-\mathrm{Li}(1)-\mathrm{Ce}(1 \mathrm{~B}) \# 3$ & $39.0(6)$ & $\operatorname{Li}(1)-\operatorname{Li}(2)-\operatorname{Li}(1) \# 13$ & $128.4(14)$ \\
\hline Li(2)\#7-Li(1)-Ce(1B)\#3 & $110.5(10)$ & $\operatorname{Li}(2) \# 15-\operatorname{Li}(2)-\operatorname{Li}(1) \# 13$ & 72.1(14) \\
\hline Li(2)\#4-Li(1)-Ce(1B)\#3 & $67.6(7)$ & $\mathrm{F}(5)-\operatorname{Li}(2)-\operatorname{Li}(1) \# 4$ & $73.8(10)$ \\
\hline $\mathrm{F}(5)-\operatorname{Li}(1)-\mathrm{Ce}(1 \mathrm{~A}) \# 3$ & $130.2(13)$ & $\mathrm{F}(3) \# 13-\operatorname{Li}(2)-\mathrm{Li}(1) \# 4$ & $80.6(12)$ \\
\hline $\mathrm{F}(2) \# 4-\mathrm{Li}(1)-\mathrm{Ce}(1 \mathrm{~A}) \# 3$ & $108.6(11)$ & $\mathrm{F}(1) \# 14-\operatorname{Li}(2)-\operatorname{Li}(1) \# 4$ & $68.1(10)$ \\
\hline $\mathrm{F}(4) \# 7-\operatorname{Li}(1)-\mathrm{Ce}(1 \mathrm{~A}) \# 3$ & $66.7(9)$ & $\mathrm{F}(3) \# 7-\operatorname{Li}(2)-\operatorname{Li}(1) \# 4$ & $169.5(15)$ \\
\hline $\mathrm{F}(1) \# 10-\mathrm{Li}(1)-\mathrm{Ce}(1 \mathrm{~A}) \# 3$ & $32.7(6)$ & $\operatorname{Li}(1)-\operatorname{Li}(2)-\operatorname{Li}(1) \# 4$ & $92.6(10)$ \\
\hline $\mathrm{Li}(1) \# 11-\mathrm{Li}(1)-\mathrm{Ce}(1 \mathrm{~A}) \# 3$ & $71.3(4)$ & $\mathrm{Li}(2) \# 15-\mathrm{Li}(2)-\mathrm{Li}(1) \# 4$ & $128.1(19)$ \\
\hline $\operatorname{Li}(2)-\operatorname{Li}(1)-\operatorname{Ce}(1 \mathrm{~A}) \# 3$ & $155.6(11)$ & Li(1)\#13-Li(2)-Li(1)\#4 & $86.5(11)$ \\
\hline $\mathrm{Li}(2) \# 12-\mathrm{Li}(1)-\mathrm{Ce}(1 \mathrm{~A}) \# 3$ & $66.1(7)$ & $\mathrm{F}(5)-\operatorname{Li}(2)-\operatorname{Li}(1) \# 7$ & $69.0(12)$ \\
\hline Li(2)\#7-Li(1)-Ce(1A)\#3 & $67.1(7)$ & $\mathrm{F}(3) \# 13-\operatorname{Li}(2)-\operatorname{Li}(1) \# 7$ & $77.6(11)$ \\
\hline $\mathrm{Li}(2) \# 4-\mathrm{Li}(1)-\mathrm{Ce}(1 \mathrm{~A}) \# 3$ & $111.5(9)$ & $\mathrm{F}(1) \# 14-\mathrm{Li}(2)-\mathrm{Li}(1) \# 7$ & $165.9(12)$ \\
\hline $\mathrm{F}(5)-\mathrm{Li}(2)-\mathrm{F}(3) \# 13$ & $115.9(17)$ & $\mathrm{F}(3) \# 7-\operatorname{Li}(2)-\operatorname{Li}(1) \# 7$ & 63.3(9) \\
\hline $\mathrm{F}(5)-\operatorname{Li}(2)-\mathrm{F}(1) \# 14$ & $125.0(14)$ & $\operatorname{Li}(1)-\operatorname{Li}(2)-\operatorname{Li}(1) \# 7$ & $89.8(12)$ \\
\hline $\mathrm{F}(3) \# 13-\mathrm{Li}(2)-\mathrm{F}(1) \# 14$ & $95.5(15)$ & $\operatorname{Li}(2) \# 15-\operatorname{Li}(2)-\operatorname{Li}(1) \# 7$ & $61.0(13)$ \\
\hline $\mathrm{F}(5)-\mathrm{Li}(2)-\mathrm{F}(3) \# 7$ & $116.4(18)$ & $\operatorname{Li}(1) \# 13-\operatorname{Li}(2)-\operatorname{Li}(1) \# 7$ & $133.1(8)$ \\
\hline $\mathrm{F}(3) \# 13-\mathrm{Li}(2)-\mathrm{F}(3) \# 7$ & $92.4(10)$ & $\operatorname{Li}(1) \# 4-\operatorname{Li}(2)-\operatorname{Li}(1) \# 7$ & $121.9(12)$ \\
\hline $\mathrm{F}(1) \# 14-\mathrm{Li}(2)-\mathrm{F}(3) \# 7$ & $105.3(14)$ & & \\
\hline $\mathrm{F}(5)-\mathrm{Li}(2)-\mathrm{Ce}(1 \mathrm{~B}) \# 4$ & $98.7(14)$ & $\mathrm{F}(5)-\mathrm{Li}(2)-\mathrm{Ce}(1 \mathrm{~A}) \# 4$ & $97.7(10)$ \\
\hline $\mathrm{F}(3) \# 13-\mathrm{Li}(2)-\mathrm{Ce}(1 \mathrm{~B}) \# 4$ & $144.7(14)$ & $\mathrm{F}(3) \# 13-\mathrm{Li}(2)-\mathrm{Ce}(1 \mathrm{~A}) \# 4$ & $122.0(14)$ \\
\hline $\mathrm{F}(1) \# 14-\mathrm{Li}(2)-\mathrm{Ce}(1 \mathrm{~B}) \# 4$ & $67.9(10)$ & $\mathrm{F}(1) \# 14-\mathrm{Li}(2)-\mathrm{Ce}(1 \mathrm{~A}) \# 4$ & $29.9(5)$ \\
\hline $\mathrm{F}(3) \# 7-\mathrm{Li}(2)-\mathrm{Ce}(1 \mathrm{~B}) \# 4$ & $64.6(9)$ & $\mathrm{F}(3) \# 7-\mathrm{Li}(2)-\mathrm{Ce}(1 \mathrm{~A}) \# 4$ & $114.0(12)$ \\
\hline $\mathrm{F}(5)-\mathrm{Li}(2)-\operatorname{Li}(1)$ & $43.5(9)$ & $\operatorname{Li}(1)-\operatorname{Li}(2)-\mathrm{Ce}(1 \mathrm{~A}) \# 4$ & $70.8(8)$ \\
\hline $\mathrm{F}(3) \# 13-\mathrm{Li}(2)-\mathrm{Li}(1)$ & $159.3(17)$ & $\mathrm{Li}(2) \# 15-\mathrm{Li}(2)-\mathrm{Ce}(1 \mathrm{~A}) \# 4$ & 132.3(17) \\
\hline $\mathrm{F}(1) \# 14-\mathrm{Li}(2)-\mathrm{Li}(1)$ & $100.1(13)$ & $\mathrm{Li}(1) \# 13-\mathrm{Li}(2)-\mathrm{Ce}(1 \mathrm{~A}) \# 4$ & $4 \quad 62.4(8)$ \\
\hline F(3)\#7-Li(2)-Li(1) & $96.6(14)$ & Li(1)\#4-Li(2)-Ce(1A)\#4 & $64.5(7)$ \\
\hline
\end{tabular}




$\begin{array}{lclc}\mathrm{Li}(1) \# 7-\mathrm{Li}(2)-\mathrm{Ce}(1 \mathrm{~A}) \# 4 & 160.3(11) & \mathrm{Li}(1) \# 7-\mathrm{F}(2)-\mathrm{Ce}(1 \mathrm{~B}) & 69.4(9) \\ \mathrm{F}(5)-\mathrm{Li}(2)-\mathrm{Ce}(1 \mathrm{~A}) & 27.7(7) & \mathrm{Li}(1) \# 8-\mathrm{F}(2)-\mathrm{Ce}(1 \mathrm{~A}) & 134.6(10) \\ \mathrm{F}(3) \# 13-\mathrm{Li}(2)-\mathrm{Ce}(1 \mathrm{~A}) & 88.4(10) & \mathrm{Li}(1) \# 7-\mathrm{F}(2)-\mathrm{Ce}(1 \mathrm{~A}) & 134.6(10) \\ \mathrm{F}(1) \# 14-\mathrm{Li}(2)-\mathrm{Ce}(1 \mathrm{~A}) & 129.7(11) & \mathrm{Li}(2) \# 12-\mathrm{F}(3)-\mathrm{Li}(2) \# 4 & 87.6(10) \\ \mathrm{F}(3) \# 7-\mathrm{Li}(2)-\mathrm{Ce}(1 \mathrm{~A}) & 124.7(13) & \mathrm{Li}(2) \# 12-\mathrm{F}(3)-\mathrm{Ce}(1 \mathrm{~A}) & 139.0(9) \\ \mathrm{Li}(1)-\mathrm{Li}(2)-\mathrm{Ce}(1 \mathrm{~A}) & 71.1(9) & \mathrm{Li}(2) \# 4-\mathrm{F}(3)-\mathrm{Ce}(1 \mathrm{~A}) & 127.2(9) \\ \mathrm{Li}(2) \# 15-\mathrm{Li}(2)-\mathrm{Ce}(1 \mathrm{~A}) & 113.8(16) & & \\ \mathrm{Li}(1) \# 13-\mathrm{Li}(2)-\mathrm{Ce}(1 \mathrm{~A}) & 146.2(11) & \mathrm{Li}(2) \# 12-\mathrm{F}(3)-\mathrm{Ce}(1 \mathrm{~B}) \# 16141.1(10) \\ \mathrm{Li}(1) \# 4-\mathrm{Li}(2)-\mathrm{Ce}(1 \mathrm{~A}) & 63.2(6) & \mathrm{Li}(2) \# 4-\mathrm{F}(3)-\mathrm{Ce}(1 \mathrm{~B}) \# 16 & 64.2(9) \\ \mathrm{Li}(1) \# 7-\mathrm{Li}(2)-\mathrm{Ce}(1 \mathrm{~A}) & 63.0(7) & \mathrm{Ce}(1 \mathrm{~A})-\mathrm{F}(3)-\mathrm{Ce}(1 \mathrm{~B}) \# 16 & 79.3(3) \\ \mathrm{Ce}(1 \mathrm{~A}) \# 4-\mathrm{Li}(2)-\mathrm{Ce}(1 \mathrm{~A}) & 111.9(6) & \mathrm{Li}(1) \# 4-\mathrm{F}(4)-\mathrm{Ce}(1 \mathrm{~B}) \# 16 & 67.7(10) \\ \mathrm{Li}(1) \# 2-\mathrm{F}(1)-\mathrm{Li}(2) \# 8 & 112.7(11) & \mathrm{Li}(1) \# 5-\mathrm{F}(4)-\mathrm{Ce}(1 \mathrm{~B}) \# 16 & 67.7(10) \\ \mathrm{Li}(1) \# 2-\mathrm{F}(1)-\mathrm{Ce}(1 \mathrm{~A}) & 120.3(8) & \mathrm{Li}(1) \# 4-\mathrm{F}(4)-\mathrm{Ce}(1 \mathrm{~A}) & 129.6(9) \\ \mathrm{Li}(2) \# 8-\mathrm{F}(1)-\mathrm{Ce}(1 \mathrm{~A}) & 124.9(8) & \mathrm{Li}(1) \# 5-\mathrm{F}(4)-\mathrm{Ce}(1 \mathrm{~A}) & 129.6(9) \\ \mathrm{Li}(1) \# 2-\mathrm{F}(1)-\mathrm{Ce}(1 \mathrm{~B}) & 113.4(8) & \mathrm{Li}(2)-\mathrm{F}(5)-\mathrm{Li}(1) & 96.7(14) \\ \mathrm{Li}(2) \# 8-\mathrm{F}(1)-\mathrm{Ce}(1 \mathrm{~B}) & 63.2(10) & \mathrm{Li}(2)-\mathrm{F}(5)-\mathrm{Ce}(1 \mathrm{~A}) & 133.3(11) \\ \mathrm{Li}(1) \# 8-\mathrm{F}(2)-\mathrm{Li}(1) \# 7 & 76.5(16) & \mathrm{Li}(1)-\mathrm{F}(5)-\mathrm{Ce}(1 \mathrm{~A}) & 129.7(10) \\ \mathrm{Li}(1) \# 8-\mathrm{F}(2)-\mathrm{Ce}(1 \mathrm{~B}) & 69.4(9) & & \\ & & & \end{array}$

Symmetry transformations used to generate equivalent atoms:
\#1 $\mathrm{x},-\mathrm{y}+1 / 2, \mathrm{z} \quad \# 2-\mathrm{x}+1, \mathrm{y}-1 / 2,-\mathrm{z}+1$
\#3 -x+1,-y+1,-z+1
\#4 -x+3/2,-y+1,z+1/2
\#5 $-\mathrm{x}+3 / 2, \mathrm{y}-1 / 2, \mathrm{z}+1 / 2 \quad$ \#6 $\quad \mathrm{x}, \mathrm{y}, \mathrm{z}-1$
$\# 7-x+3 / 2,-y+1, z-1 / 2$
\#8 - $\mathrm{x}+3 / 2, \mathrm{y}-1 / 2, \mathrm{z}-1 / 2$
\#9 $\quad \mathrm{x},-\mathrm{y}+1 / 2, \mathrm{z}-1 \quad \# 10-\mathrm{x}+1, \mathrm{y}+1 / 2,-\mathrm{z}+1$
$\# 11 \mathrm{x},-\mathrm{y}+3 / 2, \mathrm{z}$
$\# 12 \mathrm{x}-1 / 2, \mathrm{y},-\mathrm{z}+3 / 2$
$\# 13 \mathrm{x}+1 / 2, \mathrm{y},-\mathrm{z}+3 / 2 \quad \# 14-\mathrm{x}+3 / 2, \mathrm{y}+1 / 2, \mathrm{z}+1 / 2 \quad \# 15-\mathrm{x}+2,-\mathrm{y}+1,-\mathrm{z}+1 \quad \# 16 \mathrm{x}, \mathrm{y}, \mathrm{z}+1$ 

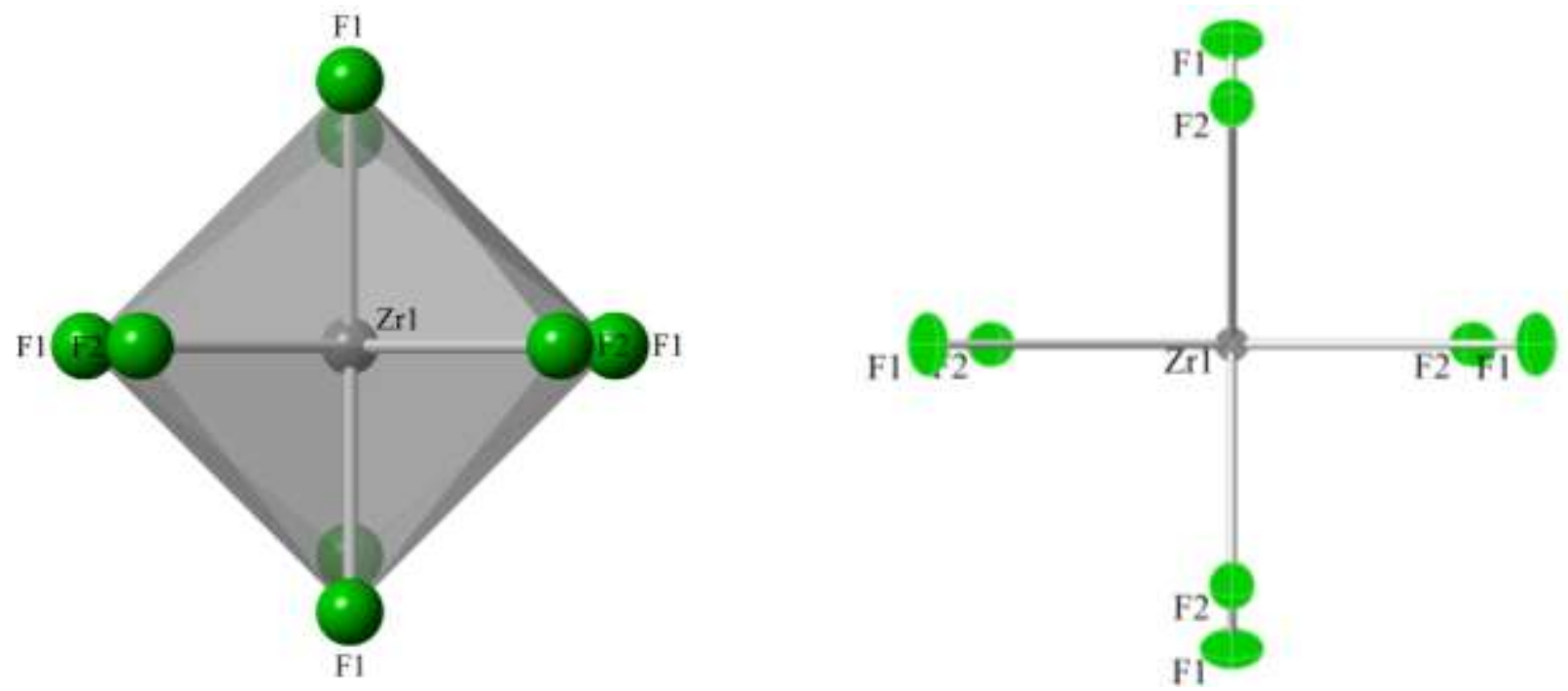

Figure S22. Local coordination environment of $\operatorname{Zr}(1)$ in $\mathbf{L i 6 B e F} 4 \mathbf{Z r F} 8$ as viewed down the primary axis of symmetry (Left) polyhedral ball and stick model (Right) thermal ellipsoid plot drawn at $50 \%$ probability with $\mathrm{Li}^{+}$and $\mathrm{Be}^{2+}$ cations omitted for clarity.

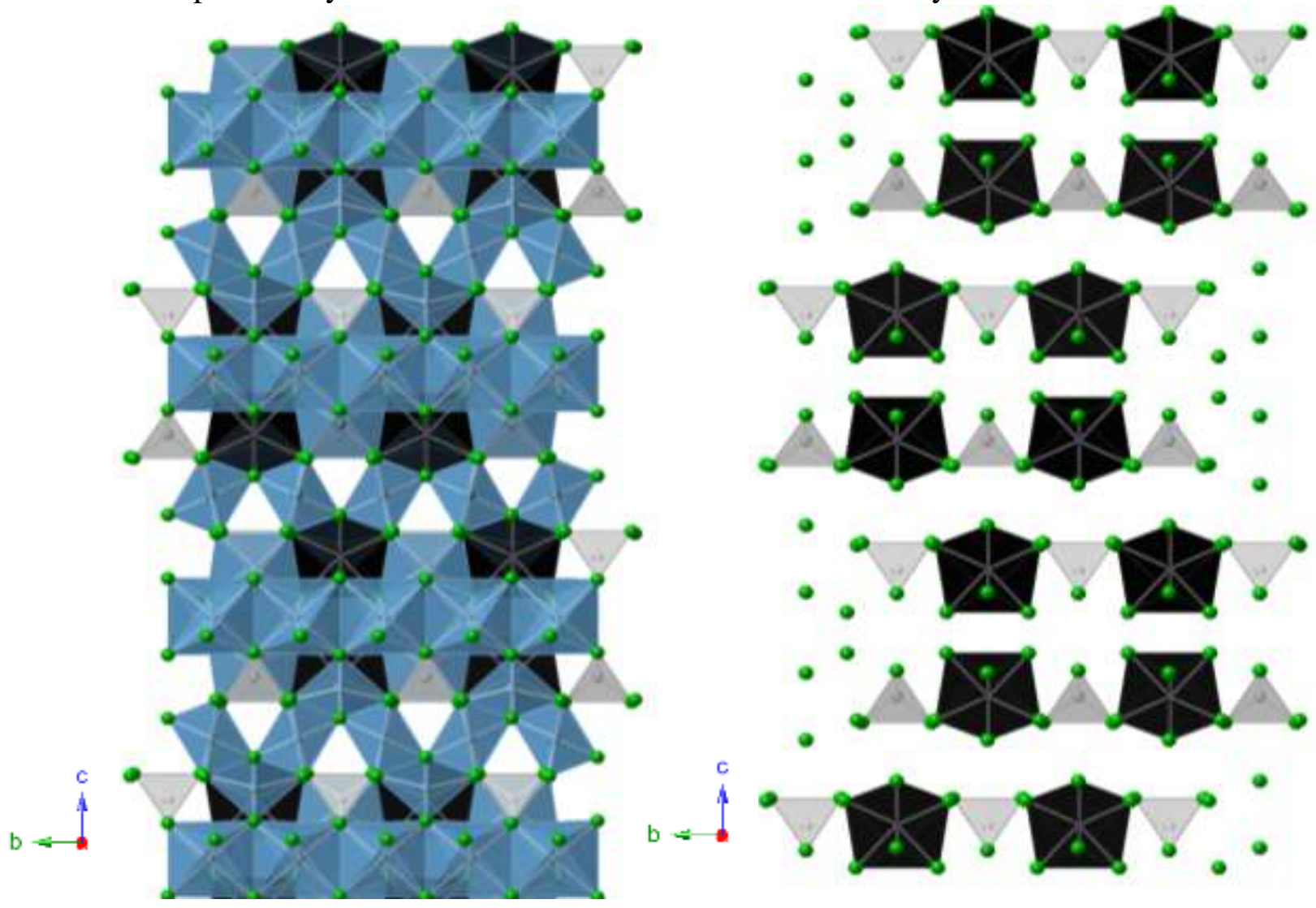

Figure S23. Extended structure view of Li6BeF4ZrF8 as viewed along the $a$-axis (Left) only $\left(\mathrm{ZrF}_{8}\right)^{4-}$ (black) and $\left(\mathrm{BeF}_{4}\right)^{2-}$ (colorless) polyhedra depicted (Right) all polyhedra represented $\mathrm{Zr}$ (black), Be (colorless), Li (blue). 
Table S4. Bond lengths $[\AA]$ and angles $\left[{ }^{\circ}\right]$ for $\operatorname{Li}_{6} \mathrm{BeF}_{4} \mathbf{Z r F}_{\mathbf{r}}$.

\begin{tabular}{|c|c|c|c|}
\hline $\mathrm{Zr}(1)-\mathrm{F}(1) \# 1$ & $2.069(1)$ & $F(2)-\operatorname{Li}(2)$ & $1.8208(8)$ \\
\hline $\mathrm{Zr}(1)-\mathrm{F}(1) \# 2$ & $2.069(1)$ & $\mathrm{F}(2)-\operatorname{Li}(2) \# 10$ & $1.8208(8)$ \\
\hline $\operatorname{Zr}(1)-\mathrm{F}(1) \# 3$ & $2.069(1)$ & $\mathrm{F}(2)-\operatorname{Li}(1) \# 11$ & $2.147(3)$ \\
\hline $\mathrm{Zr}(1)-\mathrm{F}(1)$ & $2.069(1)$ & $\mathrm{F}(3)-\mathrm{Be}(1)$ & $1.5733(9)$ \\
\hline $\mathrm{Zr}(1)-\mathrm{F}(2) \# 4$ & 2.171(1) & $\mathrm{F}(3)-\mathrm{Li}(1)$ & $2.091(2)$ \\
\hline $\mathrm{Zr}(1)-\mathrm{F}(2) \# 5$ & 2.171(1) & $\mathrm{F}(3)-\operatorname{Li}(2) \# 11$ & $2.135(3)$ \\
\hline $\mathrm{Zr}(1)-\mathrm{F}(2) \# 6$ & 2.171(1) & $\mathrm{F}(3)-\mathrm{Li}(2)$ & $2.135(3)$ \\
\hline $\mathrm{Zr}(1)-\mathrm{F}(2) \# 7$ & 2.171(1) & $\operatorname{Li}(1)-\operatorname{Li}(2) \# 7$ & $2.973(4)$ \\
\hline $\operatorname{Zr}(1)-\operatorname{Li}(1) \# 2$ & $3.319(1)$ & $\operatorname{Li}(1)-\operatorname{Li}(2) \# 11$ & $2.973(4)$ \\
\hline $\operatorname{Zr}(1)-\operatorname{Li}(1) \# 8$ & $3.319(1)$ & $\operatorname{Li}(1)-\operatorname{Li}(2) \# 12$ & $2.973(4)$ \\
\hline $\operatorname{Zr}(1)-\operatorname{Li}(1) \# 9$ & $3.319(1)$ & $\operatorname{Li}(1)-\operatorname{Li}(2)$ & $2.973(4)$ \\
\hline $\operatorname{Zr}(1)-\operatorname{Li}(1)$ & $3.319(1)$ & $\operatorname{Li}(2)-\operatorname{Li}(2) \# 11$ & $3.151(8)$ \\
\hline $\mathrm{F}(1)-\operatorname{Li}(1)$ & 1.904(3) & $\operatorname{Li}(2)-\operatorname{Li}(2) \# 7$ & $3.293(1)$ \\
\hline $\mathrm{F}(1)-\operatorname{Li}(2) \# 2$ & $2.265(3)$ & $\operatorname{Li}(2)-\operatorname{Li}(2) \# 10$ & $3.293(1)$ \\
\hline $\mathrm{F}(1)-\operatorname{Li}(2) \# 6$ & $2.265(3)$ & & \\
\hline $\mathrm{F}(1) \# 1-\mathrm{Zr}(1)-\mathrm{F}(1) \# 2$ & $130.66(5)$ & $F(1)-Z r(1)-F(2) \# 6$ & $72.34(2)$ \\
\hline $\mathrm{F}(1) \# 1-\mathrm{Zr}(1)-\mathrm{F}(1) \# 3$ & $100.03(2)$ & $\mathrm{F}(2) \# 4-\mathrm{Zr}(1)-\mathrm{F}(2) \# 6$ & $86.74(5)$ \\
\hline $\mathrm{F}(1) \# 2-\mathrm{Zr}(1)-\mathrm{F}(1) \# 3$ & $100.03(2)$ & $\mathrm{F}(2) \# 5-\mathrm{Zr}(1)-\mathrm{F}(2) \# 6$ & $121.90(3)$ \\
\hline $\mathrm{F}(1) \# 1-\mathrm{Zr}(1)-\mathrm{F}(1)$ & $100.03(2)$ & $\mathrm{F}(1) \# 1-\mathrm{Zr}(1)-\mathrm{F}(2) \# 7$ & $72.34(2)$ \\
\hline $\mathrm{F}(1) \# 2-\mathrm{Zr}(1)-\mathrm{F}(1)$ & $100.03(2)$ & $\mathrm{F}(1) \# 2-\mathrm{Zr}(1)-\mathrm{F}(2) \# 7$ & $72.34(2)$ \\
\hline $\mathrm{F}(1) \# 3-\mathrm{Zr}(1)-\mathrm{F}(1)$ & $130.66(5)$ & $\mathrm{F}(1) \# 3-\mathrm{Zr}(1)-\mathrm{F}(2) \# 7$ & $158.04(3)$ \\
\hline $\mathrm{F}(1) \# 1-\mathrm{Zr}(1)-\mathrm{F}(2) \# 4$ & $158.04(3)$ & $\mathrm{F}(1)-\mathrm{Zr}(1)-\mathrm{F}(2) \# 7$ & $71.30(4)$ \\
\hline $\mathrm{F}(1) \# 2-\mathrm{Zr}(1)-\mathrm{F}(2) \# 4$ & 71.30(4) & $\mathrm{F}(2) \# 4-\mathrm{Zr}(1)-\mathrm{F}(2) \# 7$ & $121.90(3)$ \\
\hline $\mathrm{F}(1) \# 3-\mathrm{Zr}(1)-\mathrm{F}(2) \# 4$ & $72.34(2)$ & $\mathrm{F}(2) \# 5-\mathrm{Zr}(1)-\mathrm{F}(2) \# 7$ & $86.74(5)$ \\
\hline $\mathrm{F}(1)-\mathrm{Zr}(1)-\mathrm{F}(2) \# 4$ & 72.34(2) & $\mathrm{F}(2) \# 6-\mathrm{Zr}(1)-\mathrm{F}(2) \# 7$ & 121.90(3) \\
\hline $\mathrm{F}(1) \# 1-\mathrm{Zr}(1)-\mathrm{F}(2) \# 5$ & $72.34(2)$ & $\mathrm{F}(1) \# 1-\mathrm{Zr}(1)-\operatorname{Li}(1) \# 2$ & $162.45(8)$ \\
\hline $\mathrm{F}(1) \# 2-\mathrm{Zr}(1)-\mathrm{F}(2) \# 5$ & $72.34(2)$ & $\mathrm{F}(1) \# 2-\operatorname{Zr}(1)-\operatorname{Li}(1) \# 2$ & $31.79(9)$ \\
\hline $\mathrm{F}(1) \# 3-\mathrm{Zr}(1)-\mathrm{F}(2) \# 5$ & 71.30(4) & $\mathrm{F}(1) \# 3-\mathrm{Zr}(1)-\operatorname{Li}(1) \# 2$ & $87.03(3)$ \\
\hline $\mathrm{F}(1)-\mathrm{Zr}(1)-\mathrm{F}(2) \# 5$ & $158.04(3)$ & $\mathrm{F}(1)-\mathrm{Zr}(1)-\operatorname{Li}(1) \# 2$ & $87.03(3)$ \\
\hline $\mathrm{F}(2) \# 4-\mathrm{Zr}(1)-\mathrm{F}(2) \# 5$ & $121.90(3)$ & $\mathrm{F}(2) \# 4-\mathrm{Zr}(1)-\mathrm{Li}(1) \# 2$ & $39.51(8)$ \\
\hline $\mathrm{F}(1) \# 1-\mathrm{Zr}(1)-\mathrm{F}(2) \# 6$ & $71.30(4)$ & $\mathrm{F}(2) \# 5-\operatorname{Zr}(1)-\operatorname{Li}(1) \# 2$ & $95.17(6)$ \\
\hline $\mathrm{F}(1) \# 2-\mathrm{Zr}(1)-\mathrm{F}(2) \# 6$ & $158.04(3)$ & $\mathrm{F}(2) \# 6-\operatorname{Zr}(1)-\operatorname{Li}(1) \# 2$ & $126.25(9)$ \\
\hline $\mathrm{F}(1) \# 3-\mathrm{Zr}(1)-\mathrm{F}(2) \# 6$ & $72.34(2)$ & $\mathrm{F}(2) \# 7-\operatorname{Zr}(1)-\operatorname{Li}(1) \# 2$ & $95.17(6)$ \\
\hline
\end{tabular}




\begin{tabular}{|c|c|c|c|}
\hline $\mathrm{F}(1) \# 1-\operatorname{Zr}(1)-\operatorname{Li}(1) \# 8$ & $87.03(3)$ & $\mathrm{Li}(2)-\mathrm{F}(2)-\mathrm{Li}(2) \# 10$ & $129.49(8)$ \\
\hline $\mathrm{F}(1) \# 2-\operatorname{Zr}(1)-\operatorname{Li}(1) \# 8$ & $87.03(3)$ & $\mathrm{Li}(2)-\mathrm{F}(2)-\mathrm{Li}(1) \# 11$ & $96.70(12)$ \\
\hline $\mathrm{F}(1) \# 3-\mathrm{Zr}(1)-\mathrm{Li}(1) \# 8$ & $31.79(9)$ & $\operatorname{Li}(2) \# 10-\mathrm{F}(2)-\operatorname{Li}(1) \# 11$ & $96.70(12)$ \\
\hline $\mathrm{F}(1)-\operatorname{Zr}(1)-\operatorname{Li}(1) \# 8$ & $162.45(9)$ & $\operatorname{Li}(2)-\mathrm{F}(2)-\operatorname{Zr}(1) \# 5$ & $112.48(9)$ \\
\hline $\mathrm{F}(2) \# 4-\mathrm{Zr}(1)-\mathrm{Li}(1) \# 8$ & $95.17(6)$ & $\mathrm{Li}(2) \# 10-\mathrm{F}(2)-\mathrm{Zr}(1) \# 5$ & $112.48(9)$ \\
\hline $\mathrm{F}(2) \# 5-\operatorname{Zr}(1)-\operatorname{Li}(1) \# 8$ & $39.51(8)$ & Li(1)\#11-F(2)-Zr(1)\#5 & $100.47(11)$ \\
\hline $\mathrm{F}(2) \# 6-\operatorname{Zr}(1)-\operatorname{Li}(1) \# 8$ & $95.17(6)$ & $\operatorname{Be}(1)-\mathrm{F}(3)-\mathrm{Li}(1)$ & $129.32(13)$ \\
\hline $\mathrm{F}(2) \# 7-\mathrm{Zr}(1)-\operatorname{Li}(1) \# 8$ & $126.25(9)$ & $\operatorname{Be}(1)-\mathrm{F}(3)-\operatorname{Li}(2) \# 11$ & $121.89(6)$ \\
\hline $\operatorname{Li}(1) \# 2-\operatorname{Zr}(1)-\operatorname{Li}(1) \# 8$ & $90.88(2)$ & $\mathrm{Li}(1)-\mathrm{F}(3)-\mathrm{Li}(2) \# 11$ & $89.43(9)$ \\
\hline $\mathrm{F}(1) \# 1-\mathrm{Zr}(1)-\operatorname{Li}(1) \# 9$ & $31.79(8)$ & $\operatorname{Be}(1)-F(3)-\operatorname{Li}(2)$ & $121.89(6)$ \\
\hline $\mathrm{F}(1) \# 2-\operatorname{Zr}(1)-\operatorname{Li}(1) \# 9$ & $162.45(9)$ & $\operatorname{Li}(1)-\mathrm{F}(3)-\operatorname{Li}(2)$ & 89.43(9) \\
\hline $\mathrm{F}(1) \# 3-\operatorname{Zr}(1)-\operatorname{Li}(1) \# 9$ & $87.03(3)$ & $\mathrm{Li}(2) \# 11-\mathrm{F}(3)-\mathrm{Li}(2)$ & $95.14(14)$ \\
\hline $\mathrm{F}(1)-\operatorname{Zr}(1)-\operatorname{Li}(1) \# 9$ & $87.03(3)$ & $\mathrm{F}(3)-\mathrm{Be}(1)-\mathrm{F}(3) \# 13$ & $110.50(3)$ \\
\hline $\mathrm{F}(2) \# 4-\mathrm{Zr}(1)-\operatorname{Li}(1) \# 9$ & $126.25(9)$ & $\mathrm{F}(3)-\mathrm{Be}(1)-\mathrm{F}(3) \# 14$ & $107.43(7)$ \\
\hline $\mathrm{F}(2) \# 5-\mathrm{Zr}(1)-\operatorname{Li}(1) \# 9$ & $95.17(6)$ & $\mathrm{F}(3) \# 13-\mathrm{Be}(1)-\mathrm{F}(3) \# 14$ & $110.50(3)$ \\
\hline $\mathrm{F}(2) \# 6-\mathrm{Zr}(1)-\operatorname{Li}(1) \# 9$ & $39.51(8)$ & $\mathrm{F}(3)-\mathrm{Be}(1)-\mathrm{F}(3) \# 9$ & $110.50(3)$ \\
\hline $\mathrm{F}(2) \# 7-\mathrm{Zr}(1)-\operatorname{Li}(1) \# 9$ & $95.17(6)$ & $\mathrm{F}(3) \# 13-\mathrm{Be}(1)-\mathrm{F}(3) \# 9$ & $107.43(7)$ \\
\hline $\mathrm{Li}(1) \# 2-\operatorname{Zr}(1)-\operatorname{Li}(1) \# 9$ & $165.76(16)$ & $\mathrm{F}(3) \# 14-\mathrm{Be}(1)-\mathrm{F}(3) \# 9$ & $110.50(3)$ \\
\hline $\operatorname{Li}(1) \# 8-\operatorname{Zr}(1)-\operatorname{Li}(1) \# 9$ & 90.88(2) & $\mathrm{F}(1)-\mathrm{Li}(1)-\mathrm{F}(1) \# 12$ & $95.9(2)$ \\
\hline $\mathrm{F}(1) \# 1-\operatorname{Zr}(1)-\operatorname{Li}(1)$ & $87.03(3)$ & $\mathrm{F}(1)-\mathrm{Li}(1)-\mathrm{F}(3) \# 12$ & $99.58(7)$ \\
\hline $\mathrm{F}(1) \# 2-\operatorname{Zr}(1)-\operatorname{Li}(1)$ & $87.03(3)$ & $\mathrm{F}(1) \# 12-\operatorname{Li}(1)-\mathrm{F}(3) \# 12$ & $99.58(7)$ \\
\hline $\mathrm{F}(1) \# 3-\operatorname{Zr}(1)-\operatorname{Li}(1)$ & $162.45(8)$ & $\mathrm{F}(1)-\mathrm{Li}(1)-\mathrm{F}(3)$ & $99.58(7)$ \\
\hline $\mathrm{F}(1)-\mathrm{Zr}(1)-\operatorname{Li}(1)$ & $31.79(9)$ & $\mathrm{F}(1) \# 12-\mathrm{Li}(1)-\mathrm{F}(3)$ & $99.58(7)$ \\
\hline $\mathrm{F}(2) \# 4-\operatorname{Zr}(1)-\operatorname{Li}(1)$ & $95.17(6)$ & $F(3) \# 12-\operatorname{Li}(1)-F(3)$ & $151.2(3)$ \\
\hline $\mathrm{F}(2) \# 5-\operatorname{Zr}(1)-\operatorname{Li}(1)$ & $126.25(9)$ & $\mathrm{F}(1)-\mathrm{Li}(1)-\mathrm{F}(2) \# 7$ & $74.96(4)$ \\
\hline $\mathrm{F}(2) \# 6-\operatorname{Zr}(1)-\operatorname{Li}(1)$ & $95.17(6)$ & $\mathrm{F}(1) \# 12-\mathrm{Li}(1)-\mathrm{F}(2) \# 7$ & $170.8(2)$ \\
\hline $\mathrm{F}(2) \# 7-\operatorname{Zr}(1)-\operatorname{Li}(1)$ & $39.51(8)$ & $\mathrm{F}(3) \# 12-\mathrm{Li}(1)-\mathrm{F}(2) \# 7$ & $82.24(9)$ \\
\hline $\mathrm{Li}(1) \# 2-\operatorname{Zr}(1)-\operatorname{Li}(1)$ & $90.88(2)$ & $\mathrm{F}(3)-\mathrm{Li}(1)-\mathrm{F}(2) \# 7$ & $82.24(9)$ \\
\hline $\operatorname{Li}(1) \# 8-\operatorname{Zr}(1)-\operatorname{Li}(1)$ & $165.76(16)$ & $\mathrm{F}(1)-\mathrm{Li}(1)-\mathrm{F}(2) \# 11$ & $170.8(2)$ \\
\hline $\operatorname{Li}(1) \# 9-\operatorname{Zr}(1)-\operatorname{Li}(1)$ & $90.88(2)$ & $\mathrm{F}(1) \# 12-\mathrm{Li}(1)-\mathrm{F}(2) \# 11$ & $74.96(4)$ \\
\hline $\mathrm{Li}(1)-\mathrm{F}(1)-\mathrm{Zr}(1)$ & $113.27(11)$ & $\mathrm{F}(3) \# 12-\mathrm{Li}(1)-\mathrm{F}(2) \# 11$ & $82.24(9)$ \\
\hline Li(1)-F(1)-Li(2)\#2 & $120.50(7)$ & $\mathrm{F}(3)-\mathrm{Li}(1)-\mathrm{F}(2) \# 11$ & $82.24(9)$ \\
\hline $\operatorname{Zr}(1)-\mathrm{F}(1)-\operatorname{Li}(2) \# 2$ & $100.07(3)$ & $\mathrm{F}(2) \# 7-\mathrm{Li}(1)-\mathrm{F}(2) \# 11$ & $114.2(2)$ \\
\hline Li(1)-F(1)-Li(2)\#6 & $120.50(7)$ & $\mathrm{F}(1)-\operatorname{Li}(1)-\operatorname{Li}(2) \# 7$ & $92.10(7)$ \\
\hline $\operatorname{Zr}(1)-\mathrm{F}(1)-\operatorname{Li}(2) \# 6$ & $100.07(3)$ & F(1)\#12-Li(1)-Li(2)\#7 & $145.45(4)$ \\
\hline Li(2)\#2-F(1)-Li(2)\#6 & $98.68(13)$ & F(3)\#12-Li(1)-Li(2)\#7 & $45.88(7)$ \\
\hline
\end{tabular}




\begin{tabular}{|c|c|c|c|}
\hline $\mathrm{F}(3)-\operatorname{Li}(1)-\operatorname{Li}(2) \# 7$ & $112.14(15)$ & $\mathrm{Li}(2)-\operatorname{Li}(1)-\operatorname{Zr}(1) \# 15$ & $127.26(11)$ \\
\hline $\mathrm{F}(2) \# 7-\mathrm{Li}(1)-\mathrm{Li}(2) \# 7$ & $37.46(7)$ & $\mathrm{F}(1)-\mathrm{Li}(1)-\mathrm{Zr}(1)$ & $34.93(4)$ \\
\hline $\mathrm{F}(2) \# 11-\mathrm{Li}(1)-\mathrm{Li}(2) \# 7$ & $95.52(16)$ & $\mathrm{F}(1) \# 12-\operatorname{Li}(1)-\mathrm{Zr}(1)$ & $130.82(19)$ \\
\hline $\mathrm{F}(1)-\mathrm{Li}(1)-\mathrm{Li}(2) \# 11$ & $145.45(4)$ & $\mathrm{F}(3) \# 12-\operatorname{Li}(1)-\mathrm{Zr}(1)$ & $91.766(6)$ \\
\hline $\mathrm{F}(1) \# 12-\mathrm{Li}(1)-\mathrm{Li}(2) \# 11$ & $92.10(7)$ & $\mathrm{F}(3)-\operatorname{Li}(1)-\mathrm{Zr}(1)$ & $91.766(6)$ \\
\hline $\mathrm{F}(3) \# 12-\mathrm{Li}(1)-\mathrm{Li}(2) \# 11$ & $112.14(15)$ & $\mathrm{F}(2) \# 7-\operatorname{Li}(1)-\mathrm{Zr}(1)$ & $40.02(4)$ \\
\hline $\mathrm{F}(3)-\mathrm{Li}(1)-\mathrm{Li}(2) \# 11$ & $45.88(7)$ & $\mathrm{F}(2) \# 11-\operatorname{Li}(1)-\mathrm{Zr}(1)$ & 154.22(19) \\
\hline $\mathrm{F}(2) \# 7-\mathrm{Li}(1)-\mathrm{Li}(2) \# 11$ & $95.52(16)$ & Li(2)\#7-Li(1)-Zr(1) & $63.50(7)$ \\
\hline $\mathrm{F}(2) \# 11-\mathrm{Li}(1)-\mathrm{Li}(2) \# 11$ & $37.46(7)$ & $\mathrm{Li}(2) \# 11-\mathrm{Li}(1)-\mathrm{Zr}(1)$ & $127.26(11)$ \\
\hline Li(2)\#7-Li(1)-Li(2)\#11 & $100.09(16)$ & $\mathrm{Li}(2) \# 12-\mathrm{Li}(1)-\mathrm{Zr}(1)$ & $127.26(11)$ \\
\hline $\mathrm{F}(1)-\mathrm{Li}(1)-\mathrm{Li}(2) \# 12$ & $145.45(4)$ & $\operatorname{Li}(2)-\operatorname{Li}(1)-\operatorname{Zr}(1)$ & $63.50(7)$ \\
\hline $\mathrm{F}(1) \# 12-\mathrm{Li}(1)-\mathrm{Li}(2) \# 12$ & $92.10(7)$ & $\operatorname{Zr}(1) \# 15-\operatorname{Li}(1)-\operatorname{Zr}(1)$ & $165.76(16)$ \\
\hline $\mathrm{F}(3) \# 12-\mathrm{Li}(1)-\mathrm{Li}(2) \# 12$ & $45.88(7)$ & $\mathrm{F}(2) \# 7-\mathrm{Li}(2)-\mathrm{F}(2)$ & $165.6(2)$ \\
\hline $\mathrm{F}(3)-\mathrm{Li}(1)-\mathrm{Li}(2) \# 12$ & $112.14(15)$ & $\mathrm{F}(2) \# 7-\mathrm{Li}(2)-\mathrm{F}(3) \# 11$ & $101.38(9)$ \\
\hline $\mathrm{F}(2) \# 7-\operatorname{Li}(1)-\mathrm{Li}(2) \# 12$ & $95.52(16)$ & $\mathrm{F}(2)-\mathrm{Li}(2)-\mathrm{F}(3) \# 11$ & $89.26(9)$ \\
\hline $\mathrm{F}(2) \# 11-\mathrm{Li}(1)-\mathrm{Li}(2) \# 12$ & $37.46(7)$ & $\mathrm{F}(2) \# 7-\mathrm{Li}(2)-\mathrm{F}(3)$ & $89.26(9)$ \\
\hline Li(2)\#7-Li(1)-Li(2)\#12 & $64.00(14)$ & $\mathrm{F}(2)-\mathrm{Li}(2)-\mathrm{F}(3)$ & $101.38(9)$ \\
\hline Li(2)\#11-Li(1)-Li(2)\#12 & $67.27(9)$ & $\mathrm{F}(3) \# 11-\mathrm{Li}(2)-\mathrm{F}(3)$ & $84.86(14)$ \\
\hline $\mathrm{F}(1)-\mathrm{Li}(1)-\mathrm{Li}(2)$ & $92.10(7)$ & $\mathrm{F}(2) \# 7-\mathrm{Li}(2)-\mathrm{F}(1) \# 16$ & $94.27(12)$ \\
\hline $\mathrm{F}(1) \# 12-\mathrm{Li}(1)-\mathrm{Li}(2)$ & $145.45(4)$ & $\mathrm{F}(2)-\mathrm{Li}(2)-\mathrm{F}(1) \# 16$ & $74.69(9)$ \\
\hline $\mathrm{F}(3) \# 12-\mathrm{Li}(1)-\mathrm{Li}(2)$ & $112.14(15)$ & $\mathrm{F}(3) \# 11-\mathrm{Li}(2)-\mathrm{F}(1) \# 16$ & $97.06(3)$ \\
\hline$F(3)-\operatorname{Li}(1)-\operatorname{Li}(2)$ & $45.88(7)$ & $\mathrm{F}(3)-\mathrm{Li}(2)-\mathrm{F}(1) \# 16$ & $175.56(6)$ \\
\hline $\mathrm{F}(2) \# 7-\mathrm{Li}(1)-\mathrm{Li}(2)$ & $37.46(7)$ & $\mathrm{F}(2) \# 7-\mathrm{Li}(2)-\mathrm{F}(1) \# 1$ & $74.69(9)$ \\
\hline $\mathrm{F}(2) \# 11-\mathrm{Li}(1)-\mathrm{Li}(2)$ & $95.52(16)$ & $\mathrm{F}(2)-\mathrm{Li}(2)-\mathrm{F}(1) \# 1$ & $94.27(12)$ \\
\hline $\mathrm{Li}(2) \# 7-\mathrm{Li}(1)-\mathrm{Li}(2)$ & $67.27(9)$ & $\mathrm{F}(3) \# 11-\mathrm{Li}(2)-\mathrm{F}(1) \# 1$ & $175.56(6)$ \\
\hline Li(2)\#11-Li(1)-Li(2) & $64.00(14)$ & $\mathrm{F}(3)-\mathrm{Li}(2)-\mathrm{F}(1) \# 1$ & $97.06(3)$ \\
\hline Li(2)\#12-Li(1)-Li(2) & $100.10(16)$ & $\mathrm{F}(1) \# 16-\mathrm{Li}(2)-\mathrm{F}(1) \# 1$ & $81.32(13)$ \\
\hline $\mathrm{F}(1)-\mathrm{Li}(1)-\mathrm{Zr}(1) \# 15$ & $130.82(19)$ & $\mathrm{F}(2) \# 7-\mathrm{Li}(2)-\mathrm{Li}(1)$ & $45.84(6)$ \\
\hline $\mathrm{F}(1) \# 12-\operatorname{Li}(1)-\mathrm{Zr}(1) \# 15$ & $34.93(4)$ & $\mathrm{F}(2)-\mathrm{Li}(2)-\mathrm{Li}(1)$ & $146.01(14)$ \\
\hline $\mathrm{F}(3) \# 12-\mathrm{Li}(1)-\mathrm{Zr}(1) \# 15$ & $91.766(6)$ & F(3)\#11-Li(2)-Li(1) & $85.91(12)$ \\
\hline $\mathrm{F}(3)-\mathrm{Li}(1)-\mathrm{Zr}(1) \# 15$ & $91.766(6)$ & $\mathrm{F}(3)-\mathrm{Li}(2)-\mathrm{Li}(1)$ & $44.69(5)$ \\
\hline $\mathrm{F}(2) \# 7-\operatorname{Li}(1)-\mathrm{Zr}(1) \# 15$ & $154.22(19)$ & $\mathrm{F}(1) \# 16-\mathrm{Li}(2)-\mathrm{Li}(1)$ & $139.29(6)$ \\
\hline $\mathrm{F}(2) \# 11-\operatorname{Li}(1)-\mathrm{Zr}(1) \# 15$ & $40.02(4)$ & $\mathrm{F}(1) \# 1-\operatorname{Li}(2)-\operatorname{Li}(1)$ & $92.63(7)$ \\
\hline Li(2)\#7-Li(1)-Zr(1)\#15 & $127.26(11)$ & $\mathrm{F}(2) \# 7-\mathrm{Li}(2)-\mathrm{Li}(1) \# 11$ & $146.01(14)$ \\
\hline Li(2)\#11-Li(1)-Zr(1)\#15 & $63.50(7)$ & $\mathrm{F}(2)-\mathrm{Li}(2)-\mathrm{Li}(1) \# 11$ & $45.84(6)$ \\
\hline Li(2)\#12-Li(1)-Zr(1)\#15 & $63.50(7)$ & $\mathrm{F}(3) \# 11-\mathrm{Li}(2)-\mathrm{Li}(1) \# 11$ & $44.69(5)$ \\
\hline
\end{tabular}




\begin{tabular}{|c|c|c|c|}
\hline $\mathrm{F}(3)-\mathrm{Li}(2)-\mathrm{Li}(1) \# 11$ & $85.91(12)$ & $\mathrm{F}(2) \# 7-\mathrm{Li}(2)-\mathrm{Li}(2) \# 10$ & $154.75(4)$ \\
\hline $\mathrm{F}(1) \# 16-\mathrm{Li}(2)-\mathrm{Li}(1) \# 11$ & $92.63(7)$ & $\mathrm{F}(2)-\mathrm{Li}(2)-\mathrm{Li}(2) \# 10$ & $25.26(4)$ \\
\hline $\mathrm{F}(1) \# 1-\mathrm{Li}(2)-\mathrm{Li}(1) \# 11$ & $139.29(6)$ & $\mathrm{F}(3) \# 11-\mathrm{Li}(2)-\mathrm{Li}(2) \# 10$ & $100.22(3)$ \\
\hline $\mathrm{Li}(1)-\mathrm{Li}(2)-\mathrm{Li}(1) \# 11$ & $116.00(14)$ & $\mathrm{F}(3)-\mathrm{Li}(2)-\mathrm{Li}(2) \# 10$ & $79.78(3)$ \\
\hline $\mathrm{F}(2) \# 7-\mathrm{Li}(2)-\mathrm{Li}(2) \# 11$ & $97.18(12)$ & $\mathrm{F}(1) \# 16-\mathrm{Li}(2)-\mathrm{Li}(2) \# 10$ & $95.92(2)$ \\
\hline $\mathrm{F}(2)-\mathrm{Li}(2)-\mathrm{Li}(2) \# 11$ & $97.18(12)$ & $\mathrm{F}(1) \# 1-\mathrm{Li}(2)-\mathrm{Li}(2) \# 10$ & $84.09(2)$ \\
\hline $\mathrm{F}(3) \# 11-\mathrm{Li}(2)-\mathrm{Li}(2) \# 11$ & $42.43(7)$ & $\mathrm{Li}(1)-\mathrm{Li}(2)-\mathrm{Li}(2) \# 10$ & $123.63(5)$ \\
\hline $\mathrm{F}(3)-\mathrm{Li}(2)-\mathrm{Li}(2) \# 11$ & $42.43(7)$ & $\mathrm{Li}(1) \# 11-\mathrm{Li}(2)-\mathrm{Li}(2) \# 10$ & $56.37(5)$ \\
\hline $\mathrm{F}(1) \# 16-\mathrm{Li}(2)-\mathrm{Li}(2) \# 11$ & $139.34(7)$ & $\mathrm{Li}(2) \# 11-\mathrm{Li}(2)-\mathrm{Li}(2) \# 10$ & 90.0 \\
\hline $\mathrm{F}(1) \# 1-\mathrm{Li}(2)-\mathrm{Li}(2) \# 11$ & $139.34(7)$ & Li(2)\#7-Li(2)-Li(2)\#10 & 180.0 \\
\hline $\mathrm{Li}(1)-\mathrm{Li}(2)-\mathrm{Li}(2) \# 11$ & $58.00(7)$ & $\mathrm{F}(2) \# 7-\mathrm{Li}(2)-\mathrm{Zr}(1) \# 5$ & $131.94(14)$ \\
\hline Li(1)\#11-Li(2)-Li(2)\#11 & $58.00(7)$ & $\mathrm{F}(2)-\mathrm{Li}(2)-\mathrm{Zr}(1) \# 5$ & $37.11(6)$ \\
\hline $\mathrm{F}(2) \# 7-\mathrm{Li}(2)-\mathrm{Li}(2) \# 7$ & $25.25(4)$ & $\mathrm{F}(3) \# 11-\mathrm{Li}(2)-\mathrm{Zr}(1) \# 5$ & $90.85(3)$ \\
\hline $\mathrm{F}(2)-\mathrm{Li}(2)-\mathrm{Li}(2) \# 7$ & $154.74(4)$ & $\mathrm{F}(3)-\mathrm{Li}(2)-\mathrm{Zr}(1) \# 5$ & $138.43(5)$ \\
\hline $\mathrm{F}(3) \# 11-\mathrm{Li}(2)-\mathrm{Li}(2) \# 7$ & $79.78(3)$ & $\mathrm{F}(1) \# 16-\mathrm{Li}(2)-\mathrm{Zr}(1) \# 5$ & $37.80(3)$ \\
\hline $\mathrm{F}(3)-\mathrm{Li}(2)-\mathrm{Li}(2) \# 7$ & $100.22(3)$ & $\mathrm{F}(1) \# 1-\mathrm{Li}(2)-\mathrm{Zr}(1) \# 5$ & $90.35(11)$ \\
\hline $\mathrm{F}(1) \# 16-\mathrm{Li}(2)-\mathrm{Li}(2) \# 7$ & $84.09(2)$ & Li(1)-Li(2)-Zr(1)\#5 & $175.30(7)$ \\
\hline $\mathrm{F}(1) \# 1-\mathrm{Li}(2)-\mathrm{Li}(2) \# 7$ & $95.92(2)$ & Li(1)\#11-Li(2)-Zr(1)\#5 & $63.33(4)$ \\
\hline Li(1)-Li(2)-Li(2)\#7 & $56.36(5)$ & Li(2)\#11-Li(2)-Zr(1)\#5 & $121.12(6)$ \\
\hline Li(1)\#11-Li(2)-Li(2)\#7 & $123.63(5)$ & Li(2)\#7-Li(2)-Zr(1)\#5 & $119.70(2)$ \\
\hline Li(2)\#11-Li(2)-Li(2)\#7 & 90.0 & $\mathrm{Li}(2) \# 10-\mathrm{Li}(2)-\operatorname{Zr}(1) \# 5$ & $60.30(2)$ \\
\hline
\end{tabular}

Symmetry transformations used to generate equivalent atoms:

$\# 1-y+5 / 4, x-3 / 4,-z+3 / 4 \quad \# 2 y+3 / 4,-x+5 / 4,-z+3 / 4 \quad \# 3-x+2,-y+1 / 2, z+0 \quad \# 4 y+3 / 4,-x+3 / 4, z+1 / 4$ \#5 $-\mathrm{x}+3 / 2,-\mathrm{y}+1 / 2,-\mathrm{z}+1 / 2 \quad \# 6 \quad-\mathrm{y}+5 / 4, \mathrm{x}-1 / 4, \mathrm{z}+1 / 4 \quad \# 7 \quad \mathrm{x}+1 / 2, \mathrm{y},-\mathrm{z}+1 / 2 \quad$ \#8 $\quad \mathrm{x}, \mathrm{y}-1, \mathrm{z}$ \#9 $\mathrm{y}-1 / 4,-\mathrm{x}+5 / 4,-\mathrm{z}+3 / 4 \quad \# 10 \mathrm{x}-1 / 2, \mathrm{y},-\mathrm{z}+1 / 2 \quad \# 11-\mathrm{x}+3 / 2,-\mathrm{y}+3 / 2,-\mathrm{z}+1 / 2 \quad \# 12-\mathrm{x}+2,-\mathrm{y}+3 / 2, \mathrm{z}+0$ $\# 13-y+5 / 4, x+1 / 4,-z+3 / 4 \quad \# 14-x+1,-y+3 / 2, z+0 \quad \# 15 x, y+1, z \quad \# 16 y+1 / 4,-x+5 / 4, z-1 / 4$ 

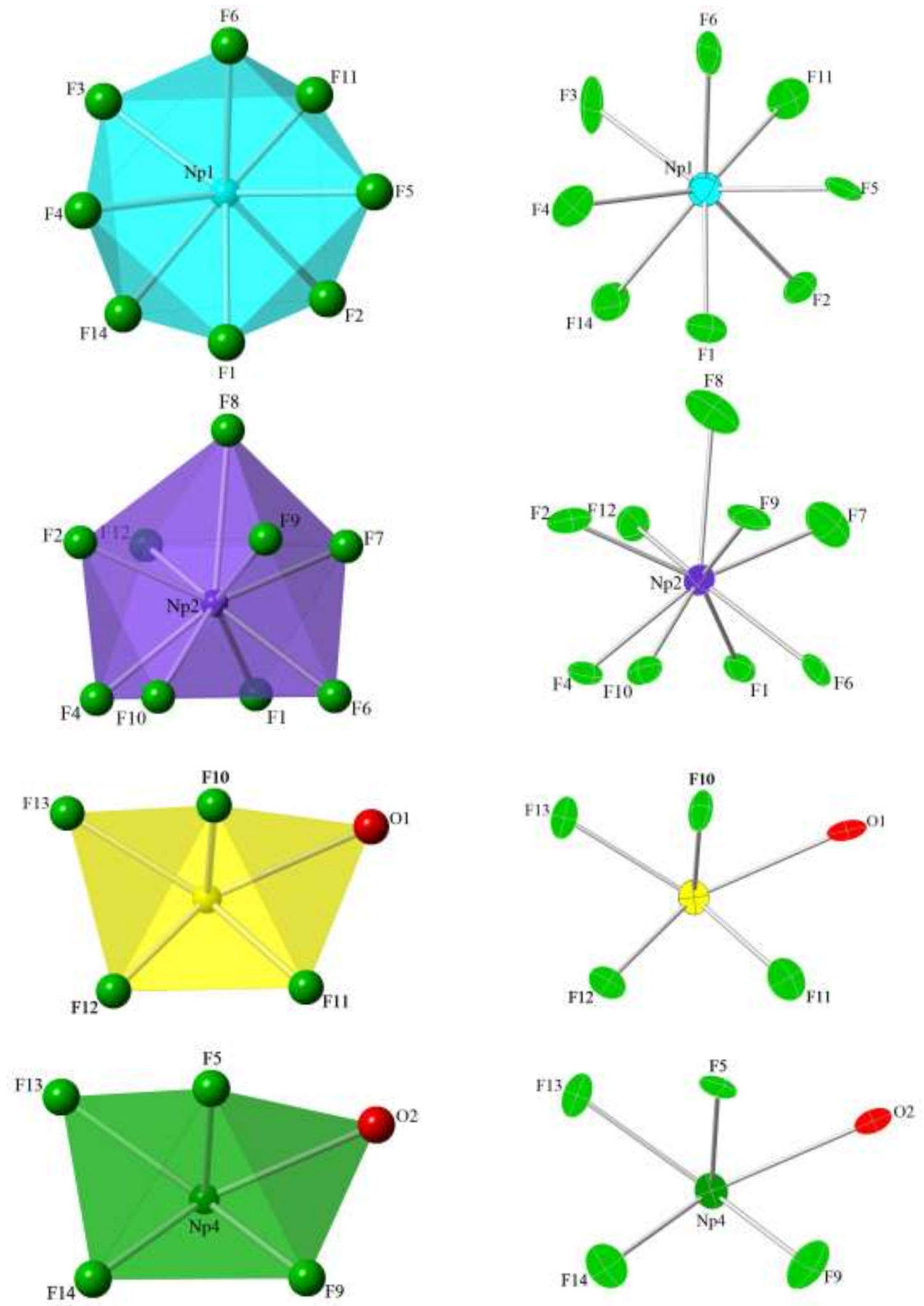

Figure S23. Local coordination environment of crystallographically unique $\mathrm{Np}$ centers in $\mathbf{N p}_{3} \mathbf{F}_{12}\left(\mathbf{H}_{2} \mathbf{O}\right)$ (Left) polyhedral ball and stick models (Right) thermal ellipsoid plots drawn at $50 \%$ probability with hydrogens omitted for clarity. 


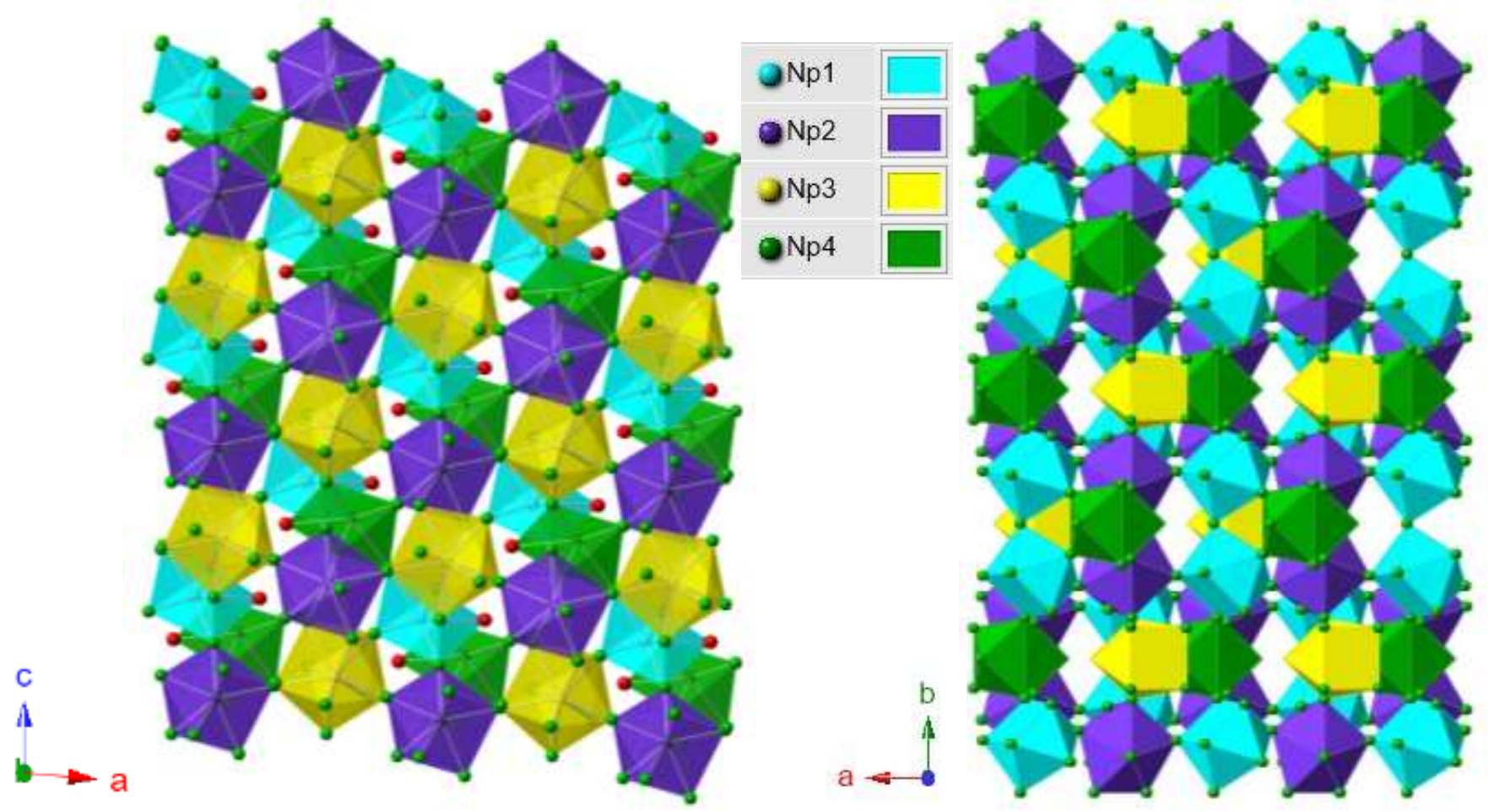

Figure S24. Extended structure views of $\mathbf{N p}_{3} \mathbf{F}_{12}\left(\mathbf{H}_{2} \mathrm{O}\right)$ drawn as ball and stick models with polyhedra drawn for the four crystallographically unique metal centers as viewed along the $b$ axis (Left) and $c$-axis (Right), with neptunium color codes shown, oxygen (red) and fluoride (green).

Table S5. Bond lengths $[\AA]$ and angles $\left[{ }^{\circ}\right]$ for $\mathbf{N p}_{3} \mathbf{F}_{12}\left(\mathbf{H}_{2} \mathbf{O}\right)$.

\begin{tabular}{llll}
\hline $\mathrm{Np}(1)-\mathrm{F}(6)$ & $2.22(4)$ & $\mathrm{Np}(2)-\mathrm{F}(12) \# 4$ & $2.29(2)$ \\
$\mathrm{Np}(1)-\mathrm{F}(5)$ & $2.22(3)$ & $\mathrm{Np}(2)-\mathrm{F}(1) \# 5$ & $2.32(3)$ \\
$\mathrm{Np}(1)-\mathrm{F}(2)$ & $2.25(3)$ & $\mathrm{Np}(2)-\mathrm{F}(6)$ & $2.30(4)$ \\
$\mathrm{Np}(1)-\mathrm{F}(11) \# 1$ & $2.26(2)$ & $\mathrm{Np}(2)-\mathrm{F}(4) \# 5$ & $2.33(3)$ \\
$\mathrm{Np}(1)-\mathrm{F}(3)$ & $2.271(9)$ & $\mathrm{Np}(2)-\mathrm{F}(10)$ & $2.34(2)$ \\
$\mathrm{Np}(1)-\mathrm{F}(14) \# 2$ & $2.29(2)$ & $\mathrm{Np}(2)-\mathrm{F}(8)$ & $2.51(2)$ \\
$\mathrm{Np}(1)-\mathrm{F}(4)$ & $2.28(3)$ & $\mathrm{Np}(2)-\mathrm{Np}(2) \# 6$ & $4.031(3)$ \\
$\mathrm{Np}(1)-\mathrm{F}(1)$ & $2.29(3)$ & & \\
$\mathrm{Np}(1)-\mathrm{Np}(2) \# 2$ & $3.851(2)$ & $\mathrm{Np}(3)-\mathrm{F}(10) \# 7$ & $2.21(3)$ \\
& & $\mathrm{Np}(3)-\mathrm{F}(10)$ & $2.21(3)$ \\
$\mathrm{Np}(2)-\mathrm{F}(9)$ & $2.23(2)$ & $\mathrm{Np}(3)-\mathrm{F}(12)$ & $2.24(2)$ \\
$\mathrm{Np}(2)-\mathrm{F}(2) \# 3$ & $2.24(3)$ & $\mathrm{Np}(3)-\mathrm{F}(12) \# 7$ & $2.24(2)$ \\
$\mathrm{Np}(2)-\mathrm{F}(7)$ & $2.27(2)$ & $\mathrm{Np}(3)-\mathrm{F}(13)$ & $2.27(5)$
\end{tabular}




\begin{tabular}{|c|c|c|c|}
\hline $\mathrm{Np}(3)-\mathrm{F}(11) \# 7$ & $2.29(2)$ & $\mathrm{Np}(4)-\mathrm{F}(5) \# 7$ & $2.27(3)$ \\
\hline $\mathrm{Np}(3)-\mathrm{F}(11)$ & $2.29(2)$ & $\mathrm{Np}(4)-\mathrm{F}(9) \# 8$ & $2.29(2)$ \\
\hline \multirow[t]{2}{*}{$\mathrm{Np}(3)-\mathrm{O}(1)$} & $2.49(5)$ & $\mathrm{Np}(4)-\mathrm{F}(9) \# 9$ & $2.29(2)$ \\
\hline & & $\mathrm{Np}(4)-\mathrm{F}(13)$ & $2.28(5)$ \\
\hline $\mathrm{Np}(4)-\mathrm{F}(14)$ & $2.23(2)$ & $\mathrm{Np}(4)-\mathrm{O}(2)$ & $2.44(5)$ \\
\hline $\mathrm{Np}(4)-\mathrm{F}(14) \# 7$ & $2.23(2)$ & $\mathrm{Np}(4)-\mathrm{F}(8) \# 9$ & $2.84(3)$ \\
\hline $\mathrm{Np}(4)-\mathrm{F}(5)$ & $2.27(3)$ & & \\
\hline $\mathrm{F}(6)-\mathrm{Np}(1)-\mathrm{F}(5)$ & $73.7(9)$ & $\mathrm{F}(6)-\mathrm{Np}(1)-\mathrm{Np}(2) \# 2$ & $97.0(8)$ \\
\hline $\mathrm{F}(6)-\mathrm{Np}(1)-\mathrm{F}(2)$ & $141.2(10)$ & $\mathrm{F}(5)-\mathrm{Np}(1)-\mathrm{Np}(2) \# 2$ & $93.6(7)$ \\
\hline $\mathrm{F}(5)-\mathrm{Np}(1)-\mathrm{F}(2)$ & $75.5(10)$ & $\mathrm{F}(2)-\mathrm{Np}(1)-\mathrm{Np}(2) \# 2$ & $108.0(7)$ \\
\hline $\mathrm{F}(6)-\mathrm{Np}(1)-\mathrm{F}(11) \# 1$ & $80.9(10)$ & $\mathrm{F}(11) \# 1-\mathrm{Np}(1)-\mathrm{Np}(2) \# 2$ & $175.9(6)$ \\
\hline $\mathrm{F}(5)-\mathrm{Np}(1)-\mathrm{F}(11) \# 1$ & $82.5(8)$ & $\mathrm{F}(3)-\mathrm{Np}(1)-\mathrm{Np}(2) \# 2$ & $108.2(7)$ \\
\hline $\mathrm{F}(2)-\mathrm{Np}(1)-\mathrm{F}(11) \# 1$ & 72.1(9) & $\mathrm{F}(14) \# 2-\mathrm{Np}(1)-\mathrm{Np}(2) \# 2$ & $70.8(6)$ \\
\hline $\mathrm{F}(6)-\mathrm{Np}(1)-\mathrm{F}(3)$ & $80.1(11)$ & $\mathrm{F}(4)-\mathrm{Np}(1)-\mathrm{Np}(2) \# 2$ & $33.7(7)$ \\
\hline $\mathrm{F}(5)-\mathrm{Np}(1)-\mathrm{F}(3)$ & $147.8(12)$ & $\mathrm{F}(1)-\mathrm{Np}(1)-\mathrm{Np}(2) \# 2$ & $33.6(8)$ \\
\hline $\mathrm{F}(2)-\mathrm{Np}(1)-\mathrm{F}(3)$ & $117.6(10)$ & & \\
\hline $\mathrm{F}(11) \# 1-\mathrm{Np}(1)-\mathrm{F}(3)$ & $75.0(9)$ & $\mathrm{F}(9)-\mathrm{Np}(2)-\mathrm{F}(2) \# 3$ & $81.5(10)$ \\
\hline $\mathrm{F}(6)-\mathrm{Np}(1)-\mathrm{F}(14) \# 2$ & 146.1(9) & $\mathrm{F}(9)-\mathrm{Np}(2)-\mathrm{F}(7)$ & $84.9(11)$ \\
\hline $\mathrm{F}(5)-\mathrm{Np}(1)-\mathrm{F}(14) \# 2$ & $136.6(9)$ & $\mathrm{F}(2) \# 3-\mathrm{Np}(2)-\mathrm{F}(7)$ & $135.5(11)$ \\
\hline $\mathrm{F}(2)-\mathrm{Np}(1)-\mathrm{F}(14) \# 2$ & $71.9(9)$ & $\mathrm{F}(9)-\mathrm{Np}(2)-\mathrm{F}(12) \# 4$ & 133.6(9) \\
\hline $\mathrm{F}(11) \# 1-\mathrm{Np}(1)-\mathrm{F}(14) \# 2$ & $112.9(8)$ & $\mathrm{F}(2) \# 3-\mathrm{Np}(2)-\mathrm{F}(12) \# 4$ & $75.4(9)$ \\
\hline $\mathrm{F}(3)-\mathrm{Np}(1)-\mathrm{F}(14) \# 2$ & $74.4(12)$ & $\mathrm{F}(7)-\mathrm{Np}(2)-\mathrm{F}(12) \# 4$ & $84.3(11)$ \\
\hline $\mathrm{F}(6)-\mathrm{Np}(1)-\mathrm{F}(4)$ & $78.8(12)$ & $\mathrm{F}(9)-\mathrm{Np}(2)-\mathrm{F}(1) \# 5$ & $144.4(10)$ \\
\hline $\mathrm{F}(5)-\mathrm{Np}(1)-\mathrm{F}(4)$ & 114.7(9) & $\mathrm{F}(2) \# 3-\mathrm{Np}(2)-\mathrm{F}(1) \# 5$ & $133.9(10)$ \\
\hline $\mathrm{F}(2)-\mathrm{Np}(1)-\mathrm{F}(4)$ & $136.5(11)$ & $\mathrm{F}(7)-\mathrm{Np}(2)-\mathrm{F}(1) \# 5$ & $71.0(11)$ \\
\hline $\mathrm{F}(11) \# 1-\mathrm{Np}(1)-\mathrm{F}(4)$ & $148.0(10)$ & $\mathrm{F}(12) \# 4-\mathrm{Np}(2)-\mathrm{F}(1) \# 5$ & $70.9(9)$ \\
\hline $\mathrm{F}(3)-\mathrm{Np}(1)-\mathrm{F}(4)$ & 77.3(9) & $\mathrm{F}(9)-\mathrm{Np}(2)-\mathrm{F}(6)$ & $74.6(10)$ \\
\hline $\mathrm{F}(14) \# 2-\mathrm{Np}(1)-\mathrm{F}(4)$ & $74.1(10)$ & $\mathrm{F}(2) \# 3-\mathrm{Np}(2)-\mathrm{F}(6)$ & $141.4(10)$ \\
\hline $\mathrm{F}(6)-\mathrm{Np}(1)-\mathrm{F}(1)$ & $116.5(10)$ & $\mathrm{F}(7)-\mathrm{Np}(2)-\mathrm{F}(6)$ & $72.5(11)$ \\
\hline $\mathrm{F}(5)-\mathrm{Np}(1)-\mathrm{F}(1)$ & $74.6(10)$ & $\mathrm{F}(12) \# 4-\mathrm{Np}(2)-\mathrm{F}(6)$ & $142.1(10)$ \\
\hline $\mathrm{F}(2)-\mathrm{Np}(1)-\mathrm{F}(1)$ & $76.5(10)$ & $\mathrm{F}(1) \# 5-\mathrm{Np}(2)-\mathrm{F}(6)$ & $73.4(11)$ \\
\hline $\mathrm{F}(11) \# 1-\mathrm{Np}(1)-\mathrm{F}(1)$ & $144.8(10)$ & $\mathrm{F}(9)-\mathrm{Np}(2)-\mathrm{F}(4) \# 5$ & $140.5(9)$ \\
\hline $\mathrm{F}(3)-\mathrm{Np}(1)-\mathrm{F}(1)$ & $135.3(11)$ & $\mathrm{F}(2) \# 3-\mathrm{Np}(2)-\mathrm{F}(4) \# 5$ & $73.1(9)$ \\
\hline $\mathrm{F}(14) \# 2-\mathrm{Np}(1)-\mathrm{F}(1)$ & $70.6(9)$ & $\mathrm{F}(7)-\mathrm{Np}(2)-\mathrm{F}(4) \# 5$ & $134.2(12)$ \\
\hline $\mathrm{F}(4)-\mathrm{Np}(1)-\mathrm{F}(1)$ & $67.1(9)$ & $\mathrm{F}(12) \# 4-\mathrm{Np}(2)-\mathrm{F}(4) \# 5$ & $68.2(9)$ \\
\hline
\end{tabular}




\begin{tabular}{|c|c|c|c|}
\hline $\mathrm{F}(1) \# 5-\mathrm{Np}(2)-\mathrm{F}(4) \# 5$ & $65.8(8)$ & & \\
\hline $\mathrm{F}(6)-\mathrm{Np}(2)-\mathrm{F}(4) \# 5$ & 107.7(9) & $\mathrm{F}(10) \# 7-\mathrm{Np}(3)-\mathrm{F}(10)$ & $108.0(15)$ \\
\hline $\mathrm{F}(9)-\mathrm{Np}(2)-\mathrm{F}(10)$ & $74.3(9)$ & $\mathrm{F}(10) \# 7-\mathrm{Np}(3)-\mathrm{F}(12)$ & $83.3(10)$ \\
\hline $\mathrm{F}(2) \# 3-\mathrm{Np}(2)-\mathrm{F}(10)$ & $72.0(8)$ & $\mathrm{F}(10)-\mathrm{Np}(3)-\mathrm{F}(12)$ & 146.3(9) \\
\hline $\mathrm{F}(7)-\mathrm{Np}(2)-\mathrm{F}(10)$ & $142.9(12)$ & $\mathrm{F}(10) \# 7-\mathrm{Np}(3)-\mathrm{F}(12) \# 7$ & $146.3(9)$ \\
\hline $\mathrm{F}(12) \# 4-\mathrm{Np}(2)-\mathrm{F}(10)$ & 132.1(9) & $\mathrm{F}(10)-\mathrm{Np}(3)-\mathrm{F}(12) \# 7$ & $83.3(10)$ \\
\hline $\mathrm{F}(1) \# 5-\mathrm{Np}(2)-\mathrm{F}(10)$ & 109.9(8) & $\mathrm{F}(12)-\mathrm{Np}(3)-\mathrm{F}(12) \# 7$ & $71.7(12)$ \\
\hline $\mathrm{F}(6)-\mathrm{Np}(2)-\mathrm{F}(10)$ & $72.5(9)$ & $\mathrm{F}(10) \# 7-\mathrm{Np}(3)-\mathrm{F}(13)$ & $74.4(10)$ \\
\hline $\mathrm{F}(4) \# 5-\mathrm{Np}(2)-\mathrm{F}(10)$ & $69.4(11)$ & $\mathrm{F}(10)-\mathrm{Np}(3)-\mathrm{F}(13)$ & $74.4(10)$ \\
\hline $\mathrm{F}(9)-\mathrm{Np}(2)-\mathrm{F}(8)$ & $66.7(9)$ & $\mathrm{F}(12)-\mathrm{Np}(3)-\mathrm{F}(13)$ & $78.7(13)$ \\
\hline $\mathrm{F}(2) \# 3-\mathrm{Np}(2)-\mathrm{F}(8)$ & $72.2(8)$ & $\mathrm{F}(12) \# 7-\mathrm{Np}(3)-\mathrm{F}(13)$ & $78.7(13)$ \\
\hline $\mathrm{F}(7)-\mathrm{Np}(2)-\mathrm{F}(8)$ & $63.4(12)$ & $\mathrm{F}(10) \# 7-\mathrm{Np}(3)-\mathrm{F}(11) \# 7$ & $140.3(9)$ \\
\hline $\mathrm{F}(12) \# 4-\mathrm{Np}(2)-\mathrm{F}(8)$ & $68.2(9)$ & $\mathrm{F}(10)-\mathrm{Np}(3)-\mathrm{F}(11) \# 7$ & $78.6(9)$ \\
\hline $\mathrm{F}(1) \# 5-\mathrm{Np}(2)-\mathrm{F}(8)$ & $120.2(8)$ & $\mathrm{F}(12)-\mathrm{Np}(3)-\mathrm{F}(11) \# 7$ & $113.2(9)$ \\
\hline $\mathrm{F}(6)-\mathrm{Np}(2)-\mathrm{F}(8)$ & $122.5(9)$ & $\mathrm{F}(12) \# 7-\mathrm{Np}(3)-\mathrm{F}(11) \# 7$ & $72.2(9)$ \\
\hline $\mathrm{F}(4) \# 5-\mathrm{Np}(2)-\mathrm{F}(8)$ & $129.4(11)$ & $\mathrm{F}(13)-\mathrm{Np}(3)-\mathrm{F}(11) \# 7$ & $142.1(7)$ \\
\hline $\mathrm{F}(10)-\mathrm{Np}(2)-\mathrm{F}(8)$ & $129.8(8)$ & $\mathrm{F}(10) \# 7-\mathrm{Np}(3)-\mathrm{F}(11)$ & $78.6(9)$ \\
\hline $\mathrm{F}(9)-\mathrm{Np}(2)-\mathrm{Np}(1) \# 5$ & $162.7(6)$ & $\mathrm{F}(10)-\mathrm{Np}(3)-\mathrm{F}(11)$ & $140.3(9)$ \\
\hline $\mathrm{F}(2) \# 3-\mathrm{Np}(2)-\mathrm{Np}(1) \# 5$ & $103.0(7)$ & $\mathrm{F}(12)-\mathrm{Np}(3)-\mathrm{F}(11)$ & $72.2(9)$ \\
\hline $\mathrm{F}(7)-\mathrm{Np}(2)-\mathrm{Np}(1) \# 5$ & $102.4(10)$ & $\mathrm{F}(12) \# 7-\mathrm{Np}(3)-\mathrm{F}(11)$ & $113.2(9)$ \\
\hline $\mathrm{F}(12) \# 4-\mathrm{Np}(2)-\mathrm{Np}(1) \# 5$ & $63.3(6)$ & $\mathrm{F}(13)-\mathrm{Np}(3)-\mathrm{F}(11)$ & $142.1(7)$ \\
\hline $\mathrm{F}(1) \# 5-\mathrm{Np}(2)-\mathrm{Np}(1) \# 5$ & $33.0(6)$ & $\mathrm{F}(11) \# 7-\mathrm{Np}(3)-\mathrm{F}(11)$ & $73.4(12)$ \\
\hline $\mathrm{F}(6)-\mathrm{Np}(2)-\mathrm{Np}(1) \# 5$ & $92.4(7)$ & $\mathrm{F}(10) \# 7-\mathrm{Np}(3)-\mathrm{O}(1)$ & $74.0(8)$ \\
\hline $\mathrm{F}(4) \# 5-\mathrm{Np}(2)-\mathrm{Np}(1) \# 5$ & $32.8(7)$ & $\mathrm{F}(10)-\mathrm{Np}(3)-\mathrm{O}(1)$ & $74.0(8)$ \\
\hline $\mathrm{F}(10)-\mathrm{Np}(2)-\mathrm{Np}(1) \# 5$ & $91.1(6)$ & $\mathrm{F}(12)-\mathrm{Np}(3)-\mathrm{O}(1)$ & $139.3(8)$ \\
\hline $\mathrm{F}(8)-\mathrm{Np}(2)-\mathrm{Np}(1) \# 5$ & $130.6(7)$ & $\mathrm{F}(12) \# 7-\mathrm{Np}(3)-\mathrm{O}(1)$ & $139.3(8)$ \\
\hline $\mathrm{F}(9)-\mathrm{Np}(2)-\mathrm{Np}(2) \# 6$ & $78.0(6)$ & $\mathrm{F}(13)-\mathrm{Np}(3)-\mathrm{O}(1)$ & $124.8(18)$ \\
\hline $\mathrm{F}(2) \# 3-\mathrm{Np}(2)-\mathrm{Np}(2) \# 6$ & 108.3(4) & $\mathrm{F}(11) \# 7-\mathrm{Np}(3)-\mathrm{O}(1)$ & $70.5(10)$ \\
\hline $\mathrm{F}(7)-\mathrm{Np}(2)-\mathrm{Np}(2) \# 6$ & $27.2(10)$ & $\mathrm{F}(11)-\mathrm{Np}(3)-\mathrm{O}(1)$ & $70.5(10)$ \\
\hline $\mathrm{F}(12) \# 4-\mathrm{Np}(2)-\mathrm{Np}(2) \# 6$ & $72.1(6)$ & & \\
\hline $\mathrm{F}(1) \# 5-\mathrm{Np}(2)-\mathrm{Np}(2) \# 6$ & $90.3(5)$ & $\mathrm{F}(14)-\mathrm{Np}(4)-\mathrm{F}(14) \# 7$ & $73.5(12)$ \\
\hline $\mathrm{F}(6)-\mathrm{Np}(2)-\mathrm{Np}(2) \# 6$ & $96.1(4)$ & $\mathrm{F}(14)-\mathrm{Np}(4)-\mathrm{F}(5)$ & $81.8(9)$ \\
\hline $\mathrm{F}(4) \# 5-\mathrm{Np}(2)-\mathrm{Np}(2) \# 6$ & $138.4(7)$ & $\mathrm{F}(14) \# 7-\mathrm{Np}(4)-\mathrm{F}(5)$ & $141.6(10)$ \\
\hline $\mathrm{F}(10)-\mathrm{Np}(2)-\mathrm{Np}(2) \# 6$ & $152.0(7)$ & $\mathrm{F}(14)-\mathrm{Np}(4)-\mathrm{F}(5) \# 7$ & $141.6(10)$ \\
\hline $\mathrm{F}(8)-\mathrm{Np}(2)-\mathrm{Np}(2) \# 6$ & $36.5(6)$ & $\mathrm{F}(14) \# 7-\mathrm{Np}(4)-\mathrm{F}(5) \# 7$ & $81.8(9)$ \\
\hline $\mathrm{Np}(1) \# 5-\mathrm{Np}(2)-\mathrm{Np}(2) \# 6$ & $115.39(3)$ & $\mathrm{F}(5)-\mathrm{Np}(4)-\mathrm{F}(5) \# 7$ & $101.5(12)$ \\
\hline
\end{tabular}




$\begin{array}{lcll}\mathrm{F}(14)-\mathrm{Np}(4)-\mathrm{F}(9) \# 8 & 76.6(9) & \mathrm{F}(5) \# 7-\mathrm{Np}(4)-\mathrm{F}(8) \# 9 & 128.7(6) \\ \mathrm{F}(14) \# 7-\mathrm{Np}(4)-\mathrm{F}(9) \# 8 & 125.5(9) & \mathrm{F}(9) \# 8-\mathrm{Np}(4)-\mathrm{F}(8) \# 9 & 60.2(8) \\ \mathrm{F}(5)-\mathrm{Np}(4)-\mathrm{F}(9) \# 8 & 74.4(9) & \mathrm{F}(9) \# 9-\mathrm{Np}(4)-\mathrm{F}(8) \# 9 & 60.2(8) \\ \mathrm{F}(5) \# 7-\mathrm{Np}(4)-\mathrm{F}(9) \# 8 & 141.4(10) & \mathrm{F}(13)-\mathrm{Np}(4)-\mathrm{F}(8) \# 9 & 130.9(16) \\ \mathrm{F}(14)-\mathrm{Np}(4)-\mathrm{F}(9) \# 9 & 125.5(9) & \mathrm{O}(2)-\mathrm{Np}(4)-\mathrm{F}(8) \# 9 & 107.9(12) \\ \mathrm{F}(14) \# 7-\mathrm{Np}(4)-\mathrm{F}(9) \# 9 & 76.6(9) & & \\ \mathrm{F}(5)-\mathrm{Np}(4)-\mathrm{F}(9) \# 9 & 141.4(10) & \mathrm{Np}(3)-\mathrm{O}(1)-\mathrm{H}(1 \mathrm{~A}) & 109.5 \\ \mathrm{~F}(5) \# 7-\mathrm{Np}(4)-\mathrm{F}(9) \# 9 & 74.4(9) & \mathrm{Np}(4)-\mathrm{O}(2)-\mathrm{H}(2 \mathrm{~A}) & 109.5 \\ \mathrm{~F}(9) \# 8-\mathrm{Np}(4)-\mathrm{F}(9) \# 9 & 85.4(12) & \mathrm{Np}(1)-\mathrm{F}(1)-\mathrm{Np}(2) \# 2 & 113.4(12) \\ \mathrm{F}(14)-\mathrm{Np}(4)-\mathrm{F}(13) & 74.9(13) & \mathrm{Np}(2) \# 10-\mathrm{F}(2)-\mathrm{Np}(1) & 148.3(8) \\ \mathrm{F}(14) \# 7-\mathrm{Np}(4)-\mathrm{F}(13) & 74.9(13) & \mathrm{Np}(1)-\mathrm{F}(3)-\mathrm{Np}(1) \# 6 & 151.5(18) \\ \mathrm{F}(5)-\mathrm{Np}(4)-\mathrm{F}(13) & 70.5(11) & \mathrm{Np}(1)-\mathrm{F}(4)-\mathrm{Np}(2) \# 2 & 113.5(12) \\ \mathrm{F}(5) \# 7-\mathrm{Np}(4)-\mathrm{F}(13) & 70.5(11) & \mathrm{Np}(1)-\mathrm{F}(5)-\mathrm{Np}(4) & 171.3(13) \\ \mathrm{F}(9) \# 8-\mathrm{Np}(4)-\mathrm{F}(13) & 137.3(6) & \mathrm{Np}(1)-\mathrm{F}(6)-\mathrm{Np}(2) & 171.8(8) \\ \mathrm{F}(9) \# 9-\mathrm{Np}(4)-\mathrm{F}(13) & 137.3(6) & \mathrm{Np}(2)-\mathrm{F}(7)-\mathrm{Np}(2) \# 6 & 126(2) \\ \mathrm{F}(14)-\mathrm{Np}(4)-\mathrm{O}(2) & 141.5(7) & \mathrm{Np}(2) \# 6-\mathrm{F}(8)-\mathrm{Np}(2) & 106.9(13) \\ \mathrm{F}(14) \# 7-\mathrm{Np}(4)-\mathrm{O}(2) & 141.5(7) & \mathrm{Np}(2) \# 6-\mathrm{F}(8)-\mathrm{Np}(4) \# 11 & 100.9(9) \\ \mathrm{F}(5)-\mathrm{Np}(4)-\mathrm{O}(2) & 73.3(9) & \mathrm{Np}(2)-\mathrm{F}(8)-\mathrm{Np}(4) \# 11 & 100.9(9) \\ \mathrm{F}(5) \# 7-\mathrm{Np}(4)-\mathrm{O}(2) & 73.3(9) & \mathrm{Np}(2)-\mathrm{F}(9)-\mathrm{Np}(4) \# 11 & 132.0(12) \\ \mathrm{F}(9) \# 8-\mathrm{Np}(4)-\mathrm{O}(2) & 68.8(9) & \mathrm{Np}(3)-\mathrm{F}(10)-\mathrm{Np}(2) & 169.3(15) \\ \mathrm{F}(9) \# 9-\mathrm{Np}(4)-\mathrm{O}(2) & 68.8(9) & \mathrm{Np}(1) \# 12-\mathrm{F}(11)-\mathrm{Np}(3) & 156.8(11) \\ \mathrm{F}(13)-\mathrm{Np}(4)-\mathrm{O}(2) & 121.3(17) & \mathrm{Np}(3)-\mathrm{F}(12)-\mathrm{Np}(2) \# 13 & 149.5(12) \\ \mathrm{F}(14)-\mathrm{Np}(4)-\mathrm{F}(8) \# 9 & 66.2(8) & \mathrm{Np}(3)-\mathrm{F}(13)-\mathrm{Np}(4) & 176(3) \\ \mathrm{F}(14) \# 7-\mathrm{Np}(4)-\mathrm{F}(8) \# 9 & 66.2(8) & \mathrm{Np}(4)-\mathrm{F}(14)-\mathrm{Np}(1) \# 5 & 156.0(12) \\ \mathrm{F}(5)-\mathrm{Np}(4)-\mathrm{F}(8) \# 9 & 128.7(6) & & \\ & & & \end{array}$

Symmetry transformations used to generate equivalent atoms:
\#1 $\mathrm{x}-1 / 2, \mathrm{y}+1 / 2, \mathrm{z}+1 / 2$
$\# 2 \mathrm{x}-1 / 2,-\mathrm{y}+3 / 2, \mathrm{z}-1 / 2$
$\# 3 \quad x+1, y, z$
$\# 4 \mathrm{x}+1 / 2, \mathrm{y}+1 / 2, \mathrm{z}+1 / 2$
\#5 $\mathrm{x}+1 / 2,-\mathrm{y}+3 / 2, \mathrm{z}+1 / 2$
\#6 $\quad x,-y+2, z$
$\# 7 \quad \mathrm{x},-\mathrm{y}+1, \mathrm{z}$
\#8 $x-1 / 2,-y+3 / 2, z+1 / 2$
\#9 $\mathrm{x}-1 / 2, \mathrm{y}-1 / 2, \mathrm{z}+1 / 2$
\#10 $\quad \mathrm{x}-1, \mathrm{y}, \mathrm{z} \quad \# 11 \quad \mathrm{x}+1 / 2, \mathrm{y}+1 / 2, \mathrm{z}-1 / 2$
$\# 12 \mathrm{x}+1 / 2, \mathrm{y}-1 / 2, \mathrm{z}-1 / 2$
$\# 13 \mathrm{x}-1 / 2, \mathrm{y}-1 / 2, \mathrm{z}-1 / 2$ 

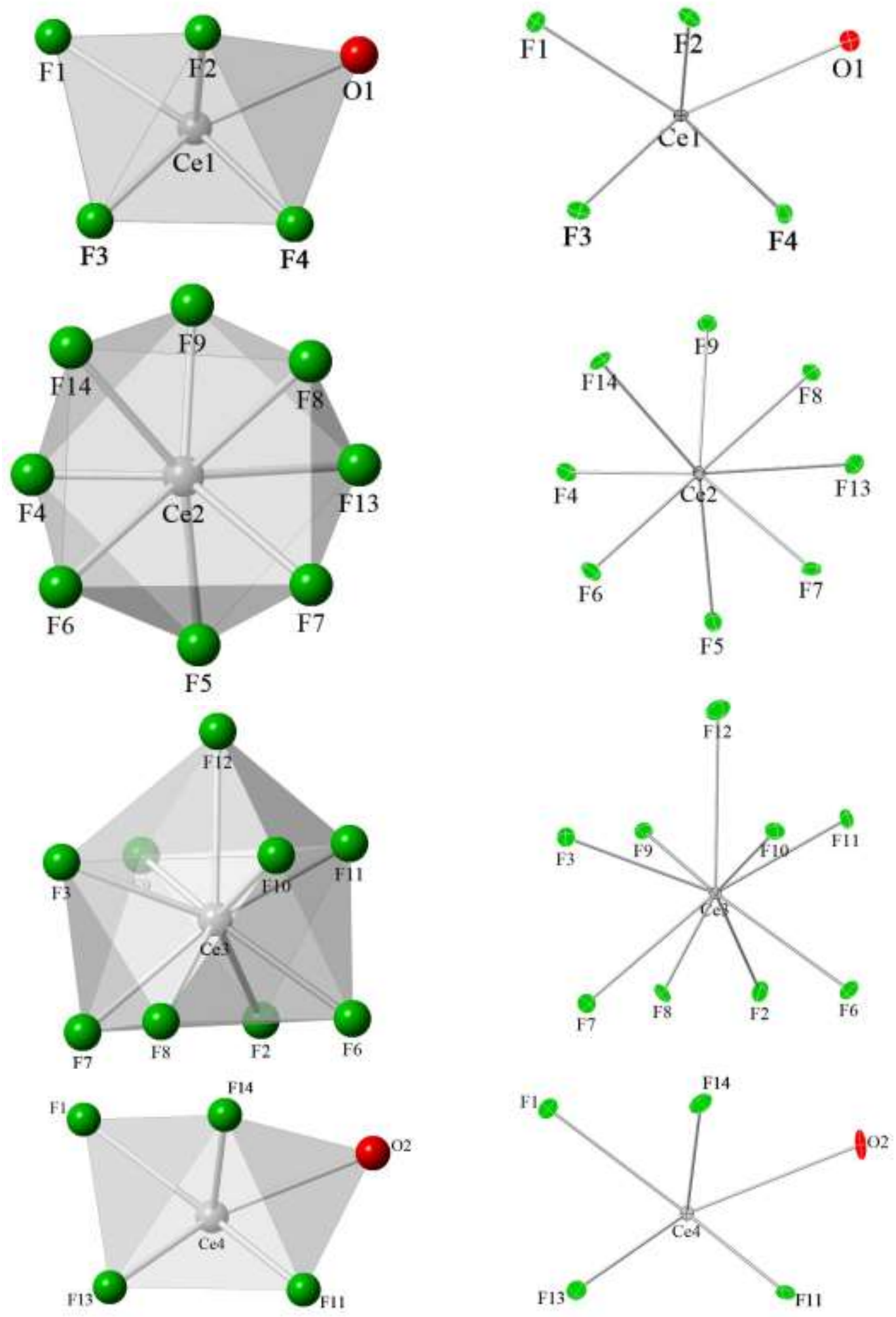

Figure S25. Local coordination environment of crystallographically unique $\mathrm{Np}$ centers in $\mathbf{N p}_{3} \mathbf{F}_{12}\left(\mathbf{H}_{2} \mathbf{O}\right)$ (Left) polyhedral ball and stick models (Right) thermal ellipsoid plots drawn at $50 \%$ probability with hydrogens omitted for clarity 

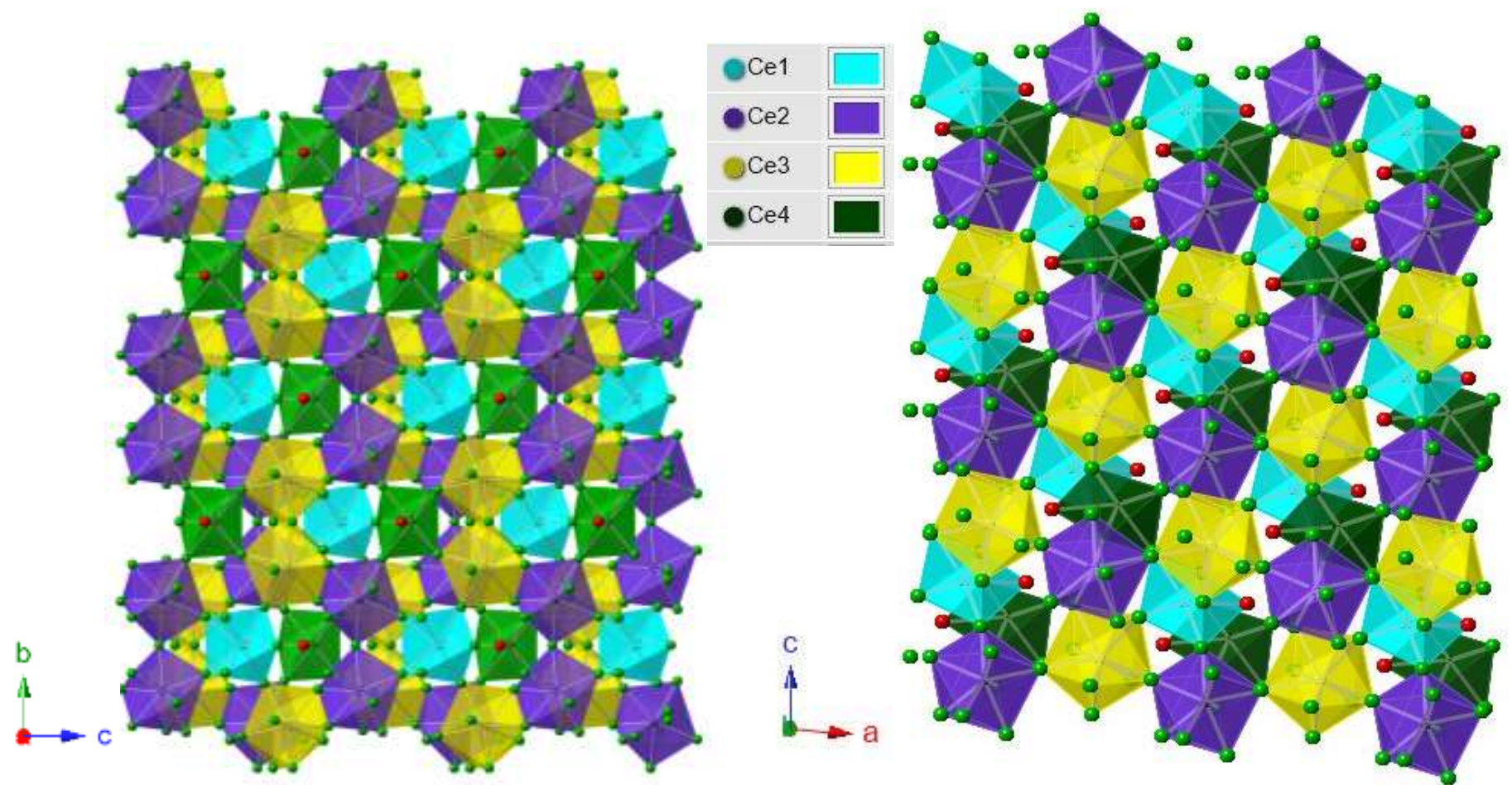

Figure S26. Extended structure views of $\mathbf{C e}_{3} \mathbf{F}_{12}\left(\mathbf{H}_{2} \mathrm{O}\right)$ drawn as ball and stick models with polyhedra drawn for the four crystallographically unique metal centers as viewed along the $a$ axis (Left) and $b$-axis (Right), with cerium color codes shown, oxygen (red) and fluoride (green).

Table S6. Bond lengths $[\AA]$ and angles $\left[{ }^{\circ}\right]$ for $\mathbf{C e}_{3} \mathbf{F}_{12}\left(\mathbf{H}_{2} \mathbf{O}\right)$.

\begin{tabular}{llll}
\hline $\mathrm{Ce}(1)-\mathrm{F}(2) \# 1$ & $2.201(3)$ & $\mathrm{Ce}(2)-\mathrm{F}(5)$ & $2.2504(9)$ \\
$\mathrm{Ce}(1)-\mathrm{F}(2)$ & $2.201(3)$ & $\mathrm{Ce}(2)-\mathrm{F}(13) \# 4$ & $2.265(3)$ \\
$\mathrm{Ce}(1)-\mathrm{F}(3) \# 1$ & $2.231(3)$ & $\mathrm{Ce}(2)-\mathrm{F}(8)$ & $2.274(3)$ \\
$\mathrm{Ce}(1)-\mathrm{F}(3)$ & $2.231(3)$ & $\mathrm{Ce}(2)-\mathrm{Ce}(3)$ & $3.8280(4)$ \\
$\mathrm{Ce}(1)-\mathrm{F}(1)$ & $2.234(5)$ & & \\
$\mathrm{Ce}(1)-\mathrm{F}(4)$ & $2.288(3)$ & $\mathrm{Ce}(3)-\mathrm{F}(9)$ & $2.215(4)$ \\
$\mathrm{Ce}(1)-\mathrm{F}(4) \# 1$ & $2.288(3)$ & $\mathrm{Ce}(3)-\mathrm{F}(11)$ & $2.216(3)$ \\
$\mathrm{Ce}(1)-\mathrm{O}(1)$ & $2.390(7)$ & $\mathrm{Ce}(3)-\mathrm{F}(10)$ & $2.251(2)$ \\
& & $\mathrm{Ce}(3)-\mathrm{F}(6) \# 6$ & $2.275(3)$ \\
$\mathrm{Ce}(2)-\mathrm{F}(6)$ & $2.204(4)$ & $\mathrm{Ce}(3)-\mathrm{F}(8)$ & $2.297(4)$ \\
$\mathrm{Ce}(2)-\mathrm{F}(4)$ & $2.218(3)$ & $\mathrm{Ce}(3)-\mathrm{F}(2) \# 7$ & $2.305(4)$ \\
$\mathrm{Ce}(2)-\mathrm{F}(14) \# 2$ & $2.233(3)$ & $\mathrm{Ce}(3)-\mathrm{F}(7)$ & $2.310(3)$ \\
$\mathrm{Ce}(2)-\mathrm{F}(7)$ & $2.246(4)$ & $\mathrm{Ce}(3)-\mathrm{F}(12)$ & $2.345(3)$ \\
$\mathrm{Ce}(2)-\mathrm{F}(9) \# 3$ & $2.248(4)$ & & $2.466(2)$
\end{tabular}




\begin{tabular}{|c|c|c|c|}
\hline $\mathrm{Ce}(3)-\mathrm{Ce}(3) \# 8$ & $4.0001(5)$ & $\mathrm{Ce}(4)-\mathrm{F}(14) \# 8$ & $2.239(3)$ \\
\hline \multirow[t]{2}{*}{$\mathrm{Ce}(3)-\mathrm{Ce}(4)$} & $4.0910(4)$ & $\mathrm{Ce}(4)-\mathrm{F}(11)$ & $2.259(3)$ \\
\hline & & $\mathrm{Ce}(4)-\mathrm{F}(11) \# 8$ & $2.259(3)$ \\
\hline $\mathrm{Ce}(4)-\mathrm{F}(13) \# 8$ & $2.207(3)$ & $\mathrm{Ce}(4)-\mathrm{F}(1) \# 9$ & $2.278(6)$ \\
\hline $\mathrm{Ce}(4)-\mathrm{F}(13)$ & $2.207(3)$ & $\mathrm{Ce}(4)-\mathrm{O}(2)$ & $2.450(6)$ \\
\hline $\mathrm{Ce}(4)-\mathrm{F}(14)$ & $2.239(3)$ & $\mathrm{Ce}(4)-\mathrm{F}(12)$ & $2.780(4)$ \\
\hline $\mathrm{F}(2) \# 1-\mathrm{Ce}(1)-\mathrm{F}(2)$ & $109.56(17)$ & $\mathrm{F}(6)-\mathrm{Ce}(2)-\mathrm{F}(4)$ & $82.09(13)$ \\
\hline $\mathrm{F}(2) \# 1-\mathrm{Ce}(1)-\mathrm{F}(3) \# 1$ & $82.49(12)$ & $\mathrm{F}(6)-\mathrm{Ce}(2)-\mathrm{F}(14) \# 2$ & $74.95(13)$ \\
\hline $\mathrm{F}(2)-\mathrm{Ce}(1)-\mathrm{F}(3) \# 1$ & $145.44(12)$ & $\mathrm{F}(4)-\mathrm{Ce}(2)-\mathrm{F}(14) \# 2$ & $83.44(11)$ \\
\hline $\mathrm{F}(2) \# 1-\mathrm{Ce}(1)-\mathrm{F}(3)$ & $145.44(12)$ & $\mathrm{F}(6)-\mathrm{Ce}(2)-\mathrm{F}(7)$ & $78.70(14)$ \\
\hline $\mathrm{F}(2)-\mathrm{Ce}(1)-\mathrm{F}(3)$ & $82.49(12)$ & $\mathrm{F}(4)-\mathrm{Ce}(2)-\mathrm{F}(7)$ & $147.42(11)$ \\
\hline $\mathrm{F}(3) \# 1-\mathrm{Ce}(1)-\mathrm{F}(3)$ & $71.46(15)$ & $\mathrm{F}(14) \# 2-\mathrm{Ce}(2)-\mathrm{F}(7)$ & $116.04(11)$ \\
\hline $\mathrm{F}(2) \# 1-\mathrm{Ce}(1)-\mathrm{F}(1)$ & $74.37(12)$ & $\mathrm{F}(6)-\mathrm{Ce}(2)-\mathrm{F}(9) \# 3$ & $142.15(13)$ \\
\hline $\mathrm{F}(2)-\mathrm{Ce}(1)-\mathrm{F}(1)$ & $74.37(12)$ & $\mathrm{F}(4)-\mathrm{Ce}(2)-\mathrm{F}(9) \# 3$ & $72.33(11)$ \\
\hline $\mathrm{F}(3) \# 1-\mathrm{Ce}(1)-\mathrm{F}(1)$ & $78.34(14)$ & $\mathrm{F}(14) \# 2-\mathrm{Ce}(2)-\mathrm{F}(9) \# 3$ & $74.81(12)$ \\
\hline $\mathrm{F}(3)-\mathrm{Ce}(1)-\mathrm{F}(1)$ & $78.34(14)$ & $\mathrm{F}(7)-\mathrm{Ce}(2)-\mathrm{F}(9) \# 3$ & $135.81(14)$ \\
\hline $\mathrm{F}(2) \# 1-\mathrm{Ce}(1)-\mathrm{F}(4)$ & $140.73(11)$ & $\mathrm{F}(6)-\mathrm{Ce}(2)-\mathrm{F}(5)$ & $81.31(12)$ \\
\hline $\mathrm{F}(2)-\mathrm{Ce}(1)-\mathrm{F}(4)$ & $78.93(11)$ & $\mathrm{F}(4)-\mathrm{Ce}(2)-\mathrm{F}(5)$ & $74.20(12)$ \\
\hline $\mathrm{F}(3) \# 1-\mathrm{Ce}(1)-\mathrm{F}(4)$ & $112.68(10)$ & $\mathrm{F}(14) \# 2-\mathrm{Ce}(2)-\mathrm{F}(5)$ & $149.33(14)$ \\
\hline $\mathrm{F}(3)-\mathrm{Ce}(1)-\mathrm{F}(4)$ & $72.24(10)$ & $\mathrm{F}(7)-\mathrm{Ce}(2)-\mathrm{F}(5)$ & $77.06(12)$ \\
\hline $\mathrm{F}(1)-\mathrm{Ce}(1)-\mathrm{F}(4)$ & $142.36(8)$ & $\mathrm{F}(9) \# 3-\mathrm{Ce}(2)-\mathrm{F}(5)$ & $116.39(12)$ \\
\hline $\mathrm{F}(2) \# 1-\mathrm{Ce}(1)-\mathrm{F}(4) \# 1$ & $78.93(11)$ & $\mathrm{F}(6)-\mathrm{Ce}(2)-\mathrm{F}(13) \# 4$ & $146.53(11)$ \\
\hline $\mathrm{F}(2)-\mathrm{Ce}(1)-\mathrm{F}(4) \# 1$ & $140.73(11)$ & $\mathrm{F}(4)-\mathrm{Ce}(2)-\mathrm{F}(13) \# 4$ & $111.52(10)$ \\
\hline $\mathrm{F}(3) \# 1-\mathrm{Ce}(1)-\mathrm{F}(4) \# 1$ & $72.24(10)$ & $\mathrm{F}(14) \# 2-\mathrm{Ce}(2)-\mathrm{F}(13) \# 4$ & $135.05(12)$ \\
\hline $\mathrm{F}(3)-\mathrm{Ce}(1)-\mathrm{F}(4) \# 1$ & $112.68(10)$ & $\mathrm{F}(7)-\mathrm{Ce}(2)-\mathrm{F}(13) \# 4$ & $74.12(12)$ \\
\hline $\mathrm{F}(1)-\mathrm{Ce}(1)-\mathrm{F}(4) \# 1$ & $142.36(8)$ & $\mathrm{F}(9) \# 3-\mathrm{Ce}(2)-\mathrm{F}(13) \# 4$ & $70.61(11)$ \\
\hline $\mathrm{F}(4)-\mathrm{Ce}(1)-\mathrm{F}(4) \# 1$ & $72.51(14)$ & $\mathrm{F}(5)-\mathrm{Ce}(2)-\mathrm{F}(13) \# 4$ & $73.93(13)$ \\
\hline $\mathrm{F}(2) \# 1-\mathrm{Ce}(1)-\mathrm{O}(1)$ & 73.91(12) & $\mathrm{F}(6)-\mathrm{Ce}(2)-\mathrm{F}(8)$ & $116.11(12)$ \\
\hline $\mathrm{F}(2)-\mathrm{Ce}(1)-\mathrm{O}(1)$ & $73.91(12)$ & $\mathrm{F}(4)-\mathrm{Ce}(2)-\mathrm{F}(8)$ & $144.89(11)$ \\
\hline $\mathrm{F}(3) \# 1-\mathrm{Ce}(1)-\mathrm{O}(1)$ & $140.13(10)$ & $\mathrm{F}(14) \# 2-\mathrm{Ce}(2)-\mathrm{F}(8)$ & $73.86(13)$ \\
\hline $\mathrm{F}(3)-\mathrm{Ce}(1)-\mathrm{O}(1)$ & $140.13(10)$ & $\mathrm{F}(7)-\mathrm{Ce}(2)-\mathrm{F}(8)$ & $67.68(11)$ \\
\hline $\mathrm{F}(1)-\mathrm{Ce}(1)-\mathrm{O}(1)$ & $123.4(2)$ & F(9)\#3-Ce(2)-F(8) & $76.03(12)$ \\
\hline $\mathrm{F}(4)-\mathrm{Ce}(1)-\mathrm{O}(1)$ & $71.99(14)$ & $\mathrm{F}(5)-\mathrm{Ce}(2)-\mathrm{F}(8)$ & $135.26(13)$ \\
\hline $\mathrm{F}(4) \# 1-\mathrm{Ce}(1)-\mathrm{O}(1)$ & $71.99(14)$ & $\mathrm{F}(13) \# 4-\mathrm{Ce}(2)-\mathrm{F}(8)$ & $70.65(12)$ \\
\hline & & $\mathrm{F}(6)-\mathrm{Ce}(2)-\mathrm{Ce}(3)$ & $96.49(10)$ \\
\hline
\end{tabular}




\begin{tabular}{|c|c|c|c|}
\hline $\mathrm{F}(4)-\mathrm{Ce}(2)-\mathrm{Ce}(3)$ & $176.65(7)$ & $\mathrm{F}(9)-\mathrm{Ce}(3)-\mathrm{F}(12)$ & $73.42(10)$ \\
\hline $\mathrm{F}(14) \# 2-\mathrm{Ce}(2)-\mathrm{Ce}(3)$ & $93.26(8)$ & $\mathrm{F}(11)-\mathrm{Ce}(3)-\mathrm{F}(12)$ & $65.52(12)$ \\
\hline $\mathrm{F}(7)-\mathrm{Ce}(2)-\mathrm{Ce}(3)$ & $34.33(8)$ & $\mathrm{F}(10)-\mathrm{Ce}(3)-\mathrm{F}(12)$ & $62.69(11)$ \\
\hline $\mathrm{F}(9) \# 3-\mathrm{Ce}(2)-\mathrm{Ce}(3)$ & $107.44(8)$ & $\mathrm{F}(3) \# 5-\mathrm{Ce}(3)-\mathrm{F}(12)$ & $69.64(12)$ \\
\hline $\mathrm{F}(5)-\mathrm{Ce}(2)-\mathrm{Ce}(3)$ & 108.64(10) & $\mathrm{F}(6) \# 6-\mathrm{Ce}(3)-\mathrm{F}(12)$ & $121.61(11)$ \\
\hline $\mathrm{F}(13) \# 4-\mathrm{Ce}(2)-\mathrm{Ce}(3)$ & $71.29(7)$ & $\mathrm{F}(8)-\mathrm{Ce}(3)-\mathrm{F}(12)$ & $120.40(12)$ \\
\hline \multirow[t]{2}{*}{$\mathrm{F}(8)-\mathrm{Ce}(2)-\mathrm{Ce}(3)$} & $33.54(9)$ & $\mathrm{F}(2) \# 7-\mathrm{Ce}(3)-\mathrm{F}(12)$ & $130.14(12)$ \\
\hline & & $\mathrm{F}(7)-\mathrm{Ce}(3)-\mathrm{F}(12)$ & $131.40(13)$ \\
\hline $\mathrm{F}(9)-\mathrm{Ce}(3)-\mathrm{F}(11)$ & $80.86(11)$ & $\mathrm{F}(9)-\mathrm{Ce}(3)-\mathrm{Ce}(2)$ & $103.38(8)$ \\
\hline $\mathrm{F}(9)-\mathrm{Ce}(3)-\mathrm{F}(10)$ & 135.91(11) & $\mathrm{F}(11)-\mathrm{Ce}(3)-\mathrm{Ce}(2)$ & $162.99(7)$ \\
\hline $\mathrm{F}(11)-\mathrm{Ce}(3)-\mathrm{F}(10)$ & $84.73(12)$ & $\mathrm{F}(10)-\mathrm{Ce}(3)-\mathrm{Ce}(2)$ & $102.40(10)$ \\
\hline $\mathrm{F}(9)-\mathrm{Ce}(3)-\mathrm{F}(3) \# 5$ & $76.66(12)$ & $\mathrm{F}(3) \# 5-\mathrm{Ce}(3)-\mathrm{Ce}(2)$ & $62.79(7)$ \\
\hline $\mathrm{F}(11)-\mathrm{Ce}(3)-\mathrm{F}(3) \# 5$ & 133.89(10) & $\mathrm{F}(6) \# 6-\mathrm{Ce}(3)-\mathrm{Ce}(2)$ & $92.17(9)$ \\
\hline $\mathrm{F}(10)-\mathrm{Ce}(3)-\mathrm{F}(3) \# 5$ & $84.26(12)$ & $\mathrm{F}(8)-\mathrm{Ce}(3)-\mathrm{Ce}(2)$ & $33.04(8)$ \\
\hline $\mathrm{F}(9)-\mathrm{Ce}(3)-\mathrm{F}(6) \# 6$ & $140.37(12)$ & $\mathrm{F}(2) \# 7-\mathrm{Ce}(3)-\mathrm{Ce}(2)$ & $91.13(8)$ \\
\hline $\mathrm{F}(11)-\mathrm{Ce}(3)-\mathrm{F}(6) \# 6$ & $75.04(12)$ & $\mathrm{F}(7)-\mathrm{Ce}(3)-\mathrm{Ce}(2)$ & $32.69(9)$ \\
\hline $\mathrm{F}(10)-\mathrm{Ce}(3)-\mathrm{F}(6) \# 6$ & $72.76(11)$ & $\mathrm{F}(12)-\mathrm{Ce}(3)-\mathrm{Ce}(2)$ & $131.48(10)$ \\
\hline $\mathrm{F}(3) \# 5-\mathrm{Ce}(3)-\mathrm{F}(6) \# 6$ & $141.64(12)$ & $\mathrm{F}(9)-\mathrm{Ce}(3)-\mathrm{Ce}(3) \# 8$ & $108.60(6)$ \\
\hline $\mathrm{F}(9)-\mathrm{Ce}(3)-\mathrm{F}(8)$ & $134.44(13)$ & $\mathrm{F}(11)-\mathrm{Ce}(3)-\mathrm{Ce}(3) \# 8$ & $77.85(7)$ \\
\hline $\mathrm{F}(11)-\mathrm{Ce}(3)-\mathrm{F}(8)$ & $144.55(12)$ & $\mathrm{F}(10)-\mathrm{Ce}(3)-\mathrm{Ce}(3) \# 8$ & 27.33(9) \\
\hline $\mathrm{F}(10)-\mathrm{Ce}(3)-\mathrm{F}(8)$ & $71.08(12)$ & $\mathrm{F}(3) \# 5-\mathrm{Ce}(3)-\mathrm{Ce}(3) \# 8$ & $72.14(7)$ \\
\hline $\mathrm{F}(3) \# 5-\mathrm{Ce}(3)-\mathrm{F}(8)$ & $70.33(12)$ & $\mathrm{F}(6) \# 6-\mathrm{Ce}(3)-\mathrm{Ce}(3) \# 8$ & $96.59(6)$ \\
\hline $\mathrm{F}(6) \# 6-\mathrm{Ce}(3)-\mathrm{F}(8)$ & $73.28(13)$ & $\mathrm{F}(8)-\mathrm{Ce}(3)-\mathrm{Ce}(3) \# 8$ & $90.36(7)$ \\
\hline $\mathrm{F}(9)-\mathrm{Ce}(3)-\mathrm{F}(2) \# 7$ & $72.09(11)$ & $\mathrm{F}(2) \# 7-\mathrm{Ce}(3)-\mathrm{Ce}(3) \# 8$ & $151.70(8)$ \\
\hline $\mathrm{F}(11)-\mathrm{Ce}(3)-\mathrm{F}(2) \# 7$ & $74.34(11)$ & $\mathrm{F}(7)-\mathrm{Ce}(3)-\mathrm{Ce}(3) \# 8$ & $138.95(9)$ \\
\hline $\mathrm{F}(10)-\mathrm{Ce}(3)-\mathrm{F}(2) \# 7$ & $142.00(14)$ & $\mathrm{F}(12)-\mathrm{Ce}(3)-\mathrm{Ce}(3) \# 8$ & $35.80(7)$ \\
\hline $\mathrm{F}(3) \# 5-\mathrm{Ce}(3)-\mathrm{F}(2) \# 7$ & $132.82(11)$ & $\mathrm{Ce}(2)-\mathrm{Ce}(3)-\mathrm{Ce}(3) \# 8$ & $115.372(5)$ \\
\hline $\mathrm{F}(6) \# 6-\mathrm{Ce}(3)-\mathrm{F}(2) \# 7$ & $71.38(12)$ & $\mathrm{F}(9)-\mathrm{Ce}(3)-\mathrm{Ce}(4)$ & $73.30(8)$ \\
\hline $\mathrm{F}(8)-\mathrm{Ce}(3)-\mathrm{F}(2) \# 7$ & $109.46(12)$ & $\mathrm{F}(11)-\mathrm{Ce}(3)-\mathrm{Ce}(4)$ & $24.14(7)$ \\
\hline $\mathrm{F}(9)-\mathrm{Ce}(3)-\mathrm{F}(7)$ & $73.73(12)$ & $\mathrm{F}(10)-\mathrm{Ce}(3)-\mathrm{Ce}(4)$ & $76.66(9)$ \\
\hline $\mathrm{F}(11)-\mathrm{Ce}(3)-\mathrm{F}(7)$ & $140.37(11)$ & $\mathrm{F}(3) \# 5-\mathrm{Ce}(3)-\mathrm{Ce}(4)$ & $109.92(7)$ \\
\hline $\mathrm{F}(10)-\mathrm{Ce}(3)-\mathrm{F}(7)$ & $134.34(13)$ & $\mathrm{F}(6) \# 6-\mathrm{Ce}(3)-\mathrm{Ce}(4)$ & $94.44(9)$ \\
\hline $\mathrm{F}(3) \# 5-\mathrm{Ce}(3)-\mathrm{F}(7)$ & $68.63(12)$ & $\mathrm{F}(8)-\mathrm{Ce}(3)-\mathrm{Ce}(4)$ & $147.58(8)$ \\
\hline $\mathrm{F}(6) \# 6-\mathrm{Ce}(3)-\mathrm{F}(7)$ & $106.54(12)$ & $\mathrm{F}(2) \# 7-\mathrm{Ce}(3)-\mathrm{Ce}(4)$ & $93.95(8)$ \\
\hline $\mathrm{F}(8)-\mathrm{Ce}(3)-\mathrm{F}(7)$ & $65.55(11)$ & $\mathrm{F}(7)-\mathrm{Ce}(3)-\mathrm{Ce}(4)$ & $146.28(9)$ \\
\hline $\mathrm{F}(2) \# 7-\mathrm{Ce}(3)-\mathrm{F}(7)$ & $69.21(13)$ & $\mathrm{F}(12)-\mathrm{Ce}(3)-\mathrm{Ce}(4)$ & $41.59(10)$ \\
\hline
\end{tabular}




\begin{tabular}{|c|c|c|c|}
\hline $\mathrm{Ce}(2)-\mathrm{Ce}(3)-\mathrm{Ce}(4)$ & $172.687(8)$ & $\mathrm{F}(11) \# 8-\mathrm{Ce}(4)-\mathrm{F}(12)$ & $59.55(9)$ \\
\hline \multirow[t]{2}{*}{$\mathrm{Ce}(3) \# 8-\mathrm{Ce}(3)-\mathrm{Ce}(4)$} & $60.732(5)$ & $\mathrm{F}(1) \# 9-\mathrm{Ce}(4)-\mathrm{F}(12)$ & $132.03(17)$ \\
\hline & & $\mathrm{O}(2)-\mathrm{Ce}(4)-\mathrm{F}(12)$ & $106.43(16)$ \\
\hline $\mathrm{F}(13) \# 8-\mathrm{Ce}(4)-\mathrm{F}(13)$ & $74.16(16)$ & $\mathrm{F}(13) \# 8-\mathrm{Ce}(4)-\mathrm{Ce}(3) \# 8$ & $101.81(7)$ \\
\hline $\mathrm{F}(13) \# 8-\mathrm{Ce}(4)-\mathrm{F}(14)$ & $143.53(12)$ & $\mathrm{F}(13)-\mathrm{Ce}(4)-\mathrm{Ce}(3) \# 8$ & $67.36(7)$ \\
\hline $\mathrm{F}(13)-\mathrm{Ce}(4)-\mathrm{F}(14)$ & $83.31(13)$ & $\mathrm{F}(14)-\mathrm{Ce}(4)-\mathrm{Ce}(3) \# 8$ & $95.25(8)$ \\
\hline $\mathrm{F}(13) \# 8-\mathrm{Ce}(4)-\mathrm{F}(14) \# 8$ & $83.31(13)$ & $\mathrm{F}(14) \# 8-\mathrm{Ce}(4)-\mathrm{Ce}(3) \# 8$ & $146.93(9)$ \\
\hline $\mathrm{F}(13)-\mathrm{Ce}(4)-\mathrm{F}(14) \# 8$ & $143.53(12)$ & $\mathrm{F}(11)-\mathrm{Ce}(4)-\mathrm{Ce}(3) \# 8$ & $75.40(7)$ \\
\hline $\mathrm{F}(14)-\mathrm{Ce}(4)-\mathrm{F}(14) \# 8$ & $99.54(16)$ & $\mathrm{F}(11) \# 8-\mathrm{Ce}(4)-\mathrm{Ce}(3) \# 8$ & $23.66(7)$ \\
\hline $\mathrm{F}(13) \# 8-\mathrm{Ce}(4)-\mathrm{F}(11)$ & $76.10(10)$ & $\mathrm{F}(1) \# 9-\mathrm{Ce}(4)-\mathrm{Ce}(3) \# 8$ & $141.72(9)$ \\
\hline $\mathrm{F}(13)-\mathrm{Ce}(4)-\mathrm{F}(11)$ & $125.35(10)$ & $\mathrm{O}(2)-\mathrm{Ce}(4)-\mathrm{Ce}(3) \# 8$ & $85.05(13)$ \\
\hline $\mathrm{F}(14)-\mathrm{Ce}(4)-\mathrm{F}(11)$ & 139.91(12) & $\mathrm{F}(12)-\mathrm{Ce}(4)-\mathrm{Ce}(3) \# 8$ & $36.08(5)$ \\
\hline $\mathrm{F}(14) \# 8-\mathrm{Ce}(4)-\mathrm{F}(11)$ & $74.24(11)$ & $\mathrm{F}(13) \# 8-\mathrm{Ce}(4)-\mathrm{Ce}(3)$ & $67.36(7)$ \\
\hline $\mathrm{F}(13) \# 8-\mathrm{Ce}(4)-\mathrm{F}(11) \# 8$ & $125.35(10)$ & $\mathrm{F}(13)-\mathrm{Ce}(4)-\mathrm{Ce}(3)$ & $101.81(7)$ \\
\hline $\mathrm{F}(13)-\mathrm{Ce}(4)-\mathrm{F}(11) \# 8$ & $76.10(10)$ & $\mathrm{F}(14)-\mathrm{Ce}(4)-\mathrm{Ce}(3)$ & $146.93(9)$ \\
\hline $\mathrm{F}(14)-\mathrm{Ce}(4)-\mathrm{F}(11) \# 8$ & $74.24(11)$ & $\mathrm{F}(14) \# 8-\mathrm{Ce}(4)-\mathrm{Ce}(3)$ & $95.25(8)$ \\
\hline $\mathrm{F}(14) \# 8-\mathrm{Ce}(4)-\mathrm{F}(11) \# 8$ & 139.91(12) & $\mathrm{F}(11)-\mathrm{Ce}(4)-\mathrm{Ce}(3)$ & $23.66(7)$ \\
\hline $\mathrm{F}(11)-\mathrm{Ce}(4)-\mathrm{F}(11) \# 8$ & $85.53(14)$ & $\mathrm{F}(11) \# 8-\mathrm{Ce}(4)-\mathrm{Ce}(3)$ & $75.40(7)$ \\
\hline $\mathrm{F}(13) \# 8-\mathrm{Ce}(4)-\mathrm{F}(1) \# 9$ & $75.40(14)$ & $\mathrm{F}(1) \# 9-\mathrm{Ce}(4)-\mathrm{Ce}(3)$ & $141.72(9)$ \\
\hline $\mathrm{F}(13)-\mathrm{Ce}(4)-\mathrm{F}(1) \# 9$ & $75.40(14)$ & $\mathrm{O}(2)-\mathrm{Ce}(4)-\mathrm{Ce}(3)$ & $85.05(13)$ \\
\hline $\mathrm{F}(14)-\mathrm{Ce}(4)-\mathrm{F}(1) \# 9$ & $71.33(13)$ & $\mathrm{F}(12)-\mathrm{Ce}(4)-\mathrm{Ce}(3)$ & $36.08(5)$ \\
\hline $\mathrm{F}(14) \# 8-\mathrm{Ce}(4)-\mathrm{F}(1) \# 9$ & $71.33(13)$ & $\mathrm{Ce}(3) \# 8-\mathrm{Ce}(4)-\mathrm{Ce}(3)$ & $58.536(9)$ \\
\hline $\mathrm{F}(11)-\mathrm{Ce}(4)-\mathrm{F}(1) \# 9$ & $137.21(7)$ & & \\
\hline $\mathrm{F}(11) \# 8-\mathrm{Ce}(4)-\mathrm{F}(1) \# 9$ & $137.21(7)$ & $\mathrm{Ce}(1)-\mathrm{F}(1)-\mathrm{Ce}(4) \# 10$ & $175.6(3)$ \\
\hline $\mathrm{F}(13) \# 8-\mathrm{Ce}(4)-\mathrm{O}(2)$ & $140.98(9)$ & $\mathrm{Ce}(1)-\mathrm{F}(2)-\mathrm{Ce}(3) \# 11$ & $170.77(18)$ \\
\hline $\mathrm{F}(13)-\mathrm{Ce}(4)-\mathrm{O}(2)$ & $140.98(9)$ & $\mathrm{Ce}(1)-\mathrm{F}(3)-\mathrm{Ce}(3) \# 3$ & $148.60(14)$ \\
\hline $\mathrm{F}(14)-\mathrm{Ce}(4)-\mathrm{O}(2)$ & $71.91(13)$ & $\mathrm{Ce}(2)-\mathrm{F}(4)-\mathrm{Ce}(1)$ & $158.51(14)$ \\
\hline $\mathrm{F}(14) \# 8-\mathrm{Ce}(4)-\mathrm{O}(2)$ & $71.91(13)$ & $\mathrm{Ce}(2)-\mathrm{F}(5)-\mathrm{Ce}(2) \# 1$ & $153.7(2)$ \\
\hline $\mathrm{F}(11)-\mathrm{Ce}(4)-\mathrm{O}(2)$ & $68.53(12)$ & $\mathrm{Ce}(2)-\mathrm{F}(6)-\mathrm{Ce}(3) \# 12$ & $171.16(13)$ \\
\hline $\mathrm{F}(11) \# 8-\mathrm{Ce}(4)-\mathrm{O}(2)$ & $68.53(12)$ & $\mathrm{Ce}(2)-\mathrm{F}(7)-\mathrm{Ce}(3)$ & $112.98(14)$ \\
\hline $\mathrm{F}(1) \# 9-\mathrm{Ce}(4)-\mathrm{O}(2)$ & $121.5(2)$ & $\mathrm{Ce}(2)-\mathrm{F}(8)-\mathrm{Ce}(3)$ & $113.42(14)$ \\
\hline $\mathrm{F}(13) \# 8-\mathrm{Ce}(4)-\mathrm{F}(12)$ & $66.83(10)$ & $\mathrm{Ce}(3)-\mathrm{F}(9)-\mathrm{Ce}(2) \# 5$ & $148.12(11)$ \\
\hline $\mathrm{F}(13)-\mathrm{Ce}(4)-\mathrm{F}(12)$ & $66.83(10)$ & $\mathrm{Ce}(3) \# 8-\mathrm{F}(10)-\mathrm{Ce}(3)$ & $125.34(18)$ \\
\hline $\mathrm{F}(14)-\mathrm{Ce}(4)-\mathrm{F}(12)$ & $129.13(8)$ & $\mathrm{Ce}(3)-\mathrm{F}(11)-\mathrm{Ce}(4)$ & $132.20(13)$ \\
\hline $\mathrm{F}(14) \# 8-\mathrm{Ce}(4)-\mathrm{F}(12)$ & $129.13(8)$ & $\mathrm{Ce}(3) \# 8-\mathrm{F}(12)-\mathrm{Ce}(3)$ & $108.39(15)$ \\
\hline $\mathrm{F}(11)-\mathrm{Ce}(4)-\mathrm{F}(12)$ & $59.55(9)$ & $\mathrm{Ce}(3) \# 8-\mathrm{F}(12)-\mathrm{Ce}(4)$ & $102.33(11)$ \\
\hline
\end{tabular}


$\mathrm{Ce}(3)-\mathrm{F}(12)-\mathrm{Ce}(4) \quad 102.33(11)$

$\mathrm{Ce}(2) \# 14-\mathrm{F}(14)-\mathrm{Ce}(4) \quad 168.88(18)$

$\mathrm{Ce}(4)-\mathrm{F}(13)-\mathrm{Ce}(2) \# 13 \quad 156.68(14)$

Symmetry transformations used to generate equivalent atoms:

\#1 $\mathrm{x},-\mathrm{y}+2, \mathrm{z} \quad \# 2 \quad \mathrm{x},-\mathrm{y}+1, \mathrm{z}+1 \quad \# 3 \quad \mathrm{x}-1 / 2,-\mathrm{y}+3 / 2, \mathrm{z}+1 / 2 \quad \# 4 \quad \mathrm{x}-1 / 2, \mathrm{y}+1 / 2, \mathrm{z}+1 / 2$

\#5 x+1/2,-y+3/2,z-1/2 \#6 x-1/2,-y+3/2,z-1/2 \#7 x,y,z-1 \#8 x,-y+1,z \#9 x+1/2,y-1/2,z-3/2

$\# 10 \mathrm{x}-1 / 2, \mathrm{y}+1 / 2, \mathrm{z}+3 / 2 \quad \# 11 \quad \mathrm{x}, \mathrm{y}, \mathrm{z}+1 \quad \# 12 \mathrm{x}+1 / 2,-\mathrm{y}+3 / 2, \mathrm{z}+1 / 2 \quad \# 13 \quad \mathrm{x}+1 / 2, \mathrm{y}-1 / 2, \mathrm{z}-1 / 2$

$\# 14 \mathrm{x},-\mathrm{y}+1, \mathrm{z}-1$

\section{REFERENCES}

1. APEX3, 2017.3-0; Bruker AXS, Inc.: Madison, WI, 2017.

2. SAINT, 8.34a; Bruker AXS, Inc: Madison, WI, 2013.

3. Sheldrick, G. M. SADABS, 2012/1; Bruker AXS, Inc: Madison, WI, 2012.

4. Sheldrick, G. M., Crystal structure refinement with SHELXL. Acta Cryst. 2015, C71, 3-8.

5. Olex2, 1.2.10; OlexSys Ltd: 2004-2018.

6. Sheldrick, G. M., SHELXT - integrated space-group and crystal-structure determination. Acta Cryst. 2015, A71, 3-8.

7. Dolomanov, O. V.; Bourhis, L. J.; Gildea, R. J.; Howard, J. A. K.; Puschmann, H., OLEX2: a complete structure solution, refinement and analysis program. J. Appl. Crystallogr. 2009, 42, 339-341.

8. Brunton, G., The Crystal Structure of LiUF5. Acta Cryst. 1966, 21, 814-817.

9. Yeon, J.; Smith, M. D.; Tapp, J.; Moller, A.; zur Loye, H. C., Mild Hydrothermal Crystal Growth, Structure, and Magnetic Properties of Ternary U(IV) Containing Fluorides: LiUF, $\mathrm{KU}_{2} \mathrm{~F}_{9}, \mathrm{~K}_{7} \mathrm{U}_{6} \mathrm{~F}_{31}, \mathrm{RbUF}_{5}, \mathrm{RbU}_{2} \mathrm{~F}_{9}$, and $\mathrm{RbU}_{3} \mathrm{~F}_{13}$. Inorg. Chem. 2014, 53, 6289-6298.

10. Brunton, G., The Crystal Structure of $\mathrm{Li}_{4} \mathrm{UF}_{8}$. J. Inorg. Nucl. Chem. 1967, 29, 1631-1636.

11. El Ghozzi, M.; Avignant, D.; Cousseins, J. C., X-ray Powder Diffraction Study of Li4 $\mathrm{TbF}_{8}$. Eur. J. Solid State Inorg. Chem. 1992, 29, 981-992.

12. Sears, D. R.; Burns, J. H., Crystal Structure of $\mathrm{Li}_{6} \mathrm{BeF}_{4} \mathrm{ZrF}_{8}$. J. Chem. Phys. 1964, 41, 34783483. 
13. Cousson, A.; Gasperin, M., Nouvelle Description du Tetrafluorure de Neptunium Hydrate, $\mathrm{Np}_{3} \mathrm{~F}_{12} \bullet \mathrm{H}_{2} \mathrm{O}$. Acta Cryst. 1985, C41, 804-805.

14. Yeon, J.; Smith, M. D.; Sefat, A. S.; Tran, T. T.; Halasyamani, P. S.; zur Loye, H. C., $\mathrm{U}_{3} \mathrm{~F}_{12}\left(\mathrm{H}_{2} \mathrm{O}\right)$, a Noncentrosymmetric Uranium(IV) Fluoride Prepared via a Convenient in situ Route That Creates $\mathrm{U}^{4+}$ Under Mild Hydrothermal Conditions. Inorg. Chem. 2013, 52, 83038305.

15. Burns, J. H.; Gordon, E. K., Refinement of the Crystal Structure of $\mathrm{Li}_{2} \mathrm{BeF}_{4}$. Acta Cryst. 1966, 20, 135-138.

16. Collins, D. M.; Mahar, M. C.; Whitehurst, F. W., Bonding in Lithium Tetrafluoroberyllate(II). Acta Cryst. 1983, B39, 303-306.

17. Hartman, P., A uniform description of phenakite type structures as superstructures of $\beta$ $\mathrm{Si}_{3} \mathrm{~N}_{4}$. Z Kristallogr. 1989, 187, 139-143.

18. Seiler, P., Estimation of Ionicity Coefficients in $\mathrm{Li}_{2} \mathrm{BeF}_{4}$ Crystals by X-ray Diffraction. Acta Cryst. 1993, B49, 223-235.

19. Carnall, W. T.; Liu, G. K.; Williams, C. W.; Reid, M. F., Analysis of the Crystal-Field Spectra of the Actinide Tetrafluorides. I. $\mathrm{UF}_{4}, \mathrm{NpF}_{4}$, and $\mathrm{PuF}_{4}$. J. Chem. Phys. 1991, 95, 7194-7203.

20. Due to the multitude of electronic states, it is difficult to assign the term symbol, therefore some states are unassigned or only collections of $\mathrm{J}$ states can be given.

21. Morss, L. R.; Edelstein, N. M.; Fuger, J., The Chemistry of the Actinide and Transactinide Elements. 4th ed.; Springer: Dordrecht, The Netherlands, 2010; Vol. 1-6.

22. Zachariasen, W., Crystal Chemical Studies of the $5 f$-Series of Elements. XII. New Compounds Representing Known Structure Types. Acta Cryst. 1949, 2, 388-390.

23. Kern, S.; Hayward, J.; Roberts, S.; Jr., J. W. R.; Rotella, F. J.; Soderholm, L.; Cort, B.; Tinkle, M.; West, M.; Hoisington, D.; Lander, G. H., Temperature variation of the structural parameters in actinide tetrafluorides. J. Chem. Phys. 1994, 101, 9333-9337.

24. Larson, A. C.; Roof, R. B., Jnr; Cromer, D. T., The Crystal Structure of UF. Acta Cryst. 1964, 17, 555-558.

25. Due to the multitude of electronic states, it is difficult to assign the term symbol, therefore some states are unassigned or only collections of $J$ states can be given. 
26. Chemey, A. T.; Celis-Barros, C.; Sperling, J. M.; Paez-Hernandez, D.; Albrecht-Schmitt, T. E., Structure, Spectroscopy, and Theoretical Analysis of Zero- and Three-Dimensional Lithium Plutonium Fluorides: $\mathrm{Li}_{4} \mathrm{PuF}_{8}$ and $\mathrm{LiPuF}_{5}$. Inorg. Chem. 2019, 58, 14790-14799.

27. Grzechnik, A.; Underwood, C. C.; Kolis, J. W.; Friese, K., Crystal Structures and Stability of $\mathrm{LiCeF}_{5}$ and $\mathrm{LiThF}_{5}$ at High Pressures: A Comparative Study of the Coordination Around the $\mathrm{Ce}^{4+}$ and $\mathrm{Th}^{4+}$ Ions. J. Fluor. Chem. 2013, 156, 124-129.

28. Chemey, A. T.; Albrecht-Schmitt, T. E., Redetermination of the Crystal Structure of TetraLithium Octa-Fluorido-Zirconate(IV), $\mathrm{Li}_{4} \mathrm{ZrF}_{8}$, From Single-Crystal X-ray Data. Acta Cryst. 2019, E75, 139-141.

29. Cousson, A.; Abazli, H.; Pages, M.; Gasperin, M., Tetrafluorure de Neptunium Hydrate, $\mathrm{Np}_{3} \mathrm{~F}_{12} \bullet \mathrm{H}_{2} \mathrm{O}$. Acta Cryst. 1983, C39, 425-427. 